\title{
ON THE UNIFORM SPREAD OF ALMOST SIMPLE LINEAR GROUPS
}

\author{
TIMOTHY C. BURNESS AND SIMON GUEST
}

\begin{abstract}
Let $G$ be a finite group, and let $k$ be a nonnegative integer. We say that $G$ has uniform spread $k$ if there exists a fixed conjugacy class $C$ in $G$ with the property that for any $k$ nontrivial elements $x_{1}, \ldots, x_{k}$ in $G$ there exists $y \in C$ such that $G=\left\langle x_{i}, y\right\rangle$ for all $i$. Further, the exact uniform spread of $G$, denoted by $u(G)$, is the largest $k$ such that $G$ has the uniform spread $k$ property. By a theorem of Breuer, Guralnick, and Kantor, $u(G) \geq 2$ for every finite simple group $G$. Here we consider the uniform spread of almost simple linear groups. Our main theorem states that if $G=\left\langle\mathrm{PSL}_{n}(q), g\right\rangle$ is almost simple, then $u(G) \geq 2$ (unless $G \cong S_{6}$ ), and we determine precisely when $u(G)$ tends to infinity as $|G|$ tends to infinity.
\end{abstract}

\section{Contents}

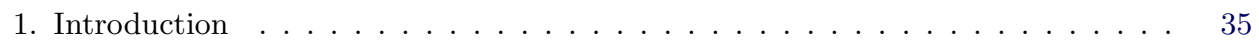

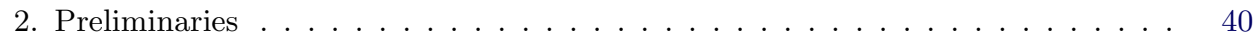

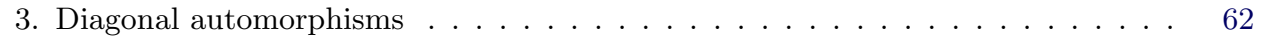

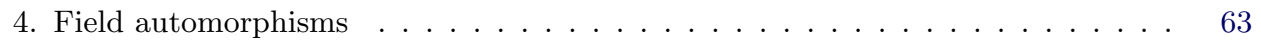

5. Graph-field automorphisms . . . . . . . . . . . . . . . . . 77

6. Graph automorphisms . . . . . . . . . . . . . . . . . . . . . 94

7. Proof of Corollary 5 . . . . . . . . . . . . . . . . . . . 105

References ............................... 106

\section{§1. Introduction}

Let $G$ be a group, and let $d(G)$ be the minimal number of generators for $G$. We say that $G$ is $d$-generated if $d(G) \leq d$. It is well known that every finite simple group is 2-generated, and in recent years a wide range of related problems on the generation of simple groups have been studied. For example, in [18], [31], and [36] it is proved that if $G$ is a finite simple group,

Received August 20, 2010. Accepted February 1, 2012.

2010 Mathematics Subject Classification. Primary 20D06; Secondary 20E28, 20F05, $20 \mathrm{P} 05$.

Burness's work partially supported by Engineering and Physical Sciences Research Council grant EP/I019545/1.

(C) 2013 by The Editorial Board of the Nagoya Mathematical Journal 
then the probability that two randomly chosen elements generate $G$ tends to 1 as $|G|$ tends to infinity, confirming a 1969 conjecture of Dixon [18, p. 205]. In a different direction, various generalizations have been investigated by imposing restrictions on the orders of the generating pairs (see, e.g., [37], [39], [40], [43]).

Following Steinberg [45], a finite group $G$ is said to be $3 / 2$-generated if every nontrivial element of $G$ belongs to a generating pair. Steinberg [45, p. 277] conjectured that every finite simple group has this strong 2generation property, and this was later proved by Guralnick and Kantor [23] using probabilistic methods. More generally, $G$ is said to have spread $k$ if, for any $k$ nontrivial elements $x_{1}, \ldots, x_{k} \in G$, there is some $y \in G$ such that $G=\left\langle x_{i}, y\right\rangle$ for all $i$. (This notion is originally due to Brenner and Wiegold [6, Definition 1.01].) We define $s(G)$ to be the exact spread of $G$, which is the largest $k$ such that $G$ has the spread $k$ property. In particular, $G$ is $3 / 2$-generated if and only if $s(G) \geq 1$.

The stronger notion of uniform spread was introduced in [23]. We say that $G$ has uniform spread $k$ if there exists a fixed conjugacy class $C$ in $G$ such that for any $k$ nontrivial elements $x_{1}, \ldots, x_{k} \in G$ there is some $y \in C$ with $G=\left\langle x_{i}, y\right\rangle$ for all $i$. We define the exact uniform spread of $G$, denoted by $u(G)$, in the obvious way. Clearly, $s(G) \geq u(G)$, and in general, these numbers are distinct. For example, if $G=\mathrm{SL}_{3}(2)$, then $s(G)=4$ and $u(G)=3$.

Let $G$ be a finite simple group. In [23] and [27] it is proved that $s(G) \geq 2$ for all but at most finitely many $G$, and that there are infinitely many examples with $s(G)=2$. This has been extended in [7], where it is proved that every finite simple group $G$ satisfies the bound $u(G) \geq 2$, with equality if and only if $G=\operatorname{Sp}_{2 m}(2)$ (with $m \geq 3$ ), $A_{5}, A_{6}$, or $\Omega_{8}^{+}(2)$ (see [7, Theorem 1.2]). Related results for almost simple groups are also obtained in [7]. (Recall that a group $G$ is almost simple if $G_{0} \leq G \leq \operatorname{Aut}\left(G_{0}\right)$ for some nonabelian finite simple group $G_{0}$, which is the socle of $G$.) Of course, if $G$ is almost simple and $G / G_{0}$ is noncyclic, then $s(G)=0$ since $G \neq\langle x, y\rangle$ for all $x \in G_{0}, y \in G$. However, the following slightly weaker spread 2 property is established in [7, Corollary 1.5]: if $x_{1}, x_{2} \in G$ are nontrivial, then there exists $y \in G$ such that $G_{0} \leq\left\langle x_{i}, y\right\rangle$ for $i=1,2$.

In this paper we consider the spread of almost simple groups. An important motivation comes from the following conjecture concerning the spread of an arbitrary finite group (see [7, Conjecture 1.8]). 
Conjecture 1. A finite group $G$ is 3/2-generated if and only if $G / N$ is cyclic for every nontrivial normal subgroup $N$ of $G$.

Clearly, the cyclic condition on quotients is necessary for 3/2-generation. For the converse, Guralnick [22] has established a reduction to the case where $G$ is almost simple with socle $G_{0}$, and some special cases have recently been established. Indeed, if $G_{0}$ is a sporadic group, then the conjecture follows from [7, Table 9 and Lemma 6.1], while the result for alternating groups follows from [7] (for $G=A_{n}$ and $G_{0}=A_{6}$ ) and [3] (for the case $\left.G=S_{n}\right)$. Therefore, to complete the proof of Conjecture 1, we may assume that $G_{0}$ is a simple group of Lie type.

The above conjecture can also be interpreted in terms of the generating graph of a finite group $G$, which is defined as follows. Let $\Gamma(G)$ be the graph defined on the set of nontrivial elements of $G$ so that two vertices $x, y$ are joined by an edge if and only if $G=\langle x, y\rangle$. Then $G$ is $3 / 2$-generated if and only if there is no isolated vertex in $\Gamma(G)$. Similarly, $G$ has spread 2 if and only if the diameter of $\Gamma(G)$ is at most 2 . An even stronger conjecture is proposed in [8, Conjecture 1.6]: if $|G| \geq 4$, then $\Gamma(G)$ contains a Hamiltonian cycle (a path that visits each vertex exactly once) if and only if $G / N$ is cyclic for every nontrivial normal subgroup $N$ of $G$; for example, it is known that all sufficiently large finite simple groups have this remarkable property (see [8, Theorem 1.2]).

The purpose of this paper is to establish a stronger version of Conjecture 1 in the case $G_{0}=\operatorname{PSL}_{n}(q)$. Our main theorem is the following.

THEOREM 2. Let $G=\left\langle\operatorname{PSL}_{n}(q), g\right\rangle$ be an almost simple group. Then either $u(G) \geq 2$, or $G=\operatorname{PSL}_{2}(9) .2 \cong S_{6}$ and $u(G)=0$.

In a spirit similar to [7] and [23], probabilistic methods play an essential role in the proof of Theorem 2 . Indeed, our main theorem is an easy corollary of Theorem 3 below on random generation. To state the result, we require some additional notation. Let $G$ be a finite group, let $C$ be a conjugacy class of $G$, and let $x \in G$. We write $\mathbb{P}(G=\langle x, y\rangle \mid y \in C)$ for the probability that $x$ and a randomly chosen element of $C$ generate $G$.

THEOREM 3. Let $G=\left\langle\operatorname{PSL}_{n}(q), g\right\rangle$ be an almost simple group. Then either there exists a $G$-class $C \subseteq g \operatorname{PSL}_{n}(q)$ such that

$$
\mathbb{P}(G=\langle x, y\rangle \mid y \in C)>1 / 2
$$

for all nontrivial $x \in G$, or 


$$
G \in\left\{\mathrm{PSL}_{2}(9) .2, \mathrm{PSL}_{3}(4) .2_{1}, \mathrm{PSL}_{4}(2) .2, \mathrm{PSL}_{4}(3) .2_{2}\right\},
$$

where $\mathrm{PSL}_{2}(9) .2 \cong S_{6}, \mathrm{PSL}_{3}(4) .2_{1}$ is an extension of $\mathrm{PSL}_{3}(4)$ by a graphfield automorphism, and $\mathrm{PSL}_{4}(3) .2_{2} \cong\left\langle\mathrm{PSL}_{4}(3), \iota\right\rangle$ where $\iota$ is the inversetranspose graph automorphism.

The groups in (1) are genuine exceptions, but in each case it is easy to check directly that $u(G) \geq 2$, unless $G=\operatorname{PSL}_{2}(9) .2$, where we have $u(G)=0$ and $s(G)=2$ (see Section 2.7). Note that $\operatorname{PSL}_{4}(2) .2 \cong S_{8}$ and $\operatorname{PSL}_{4}(3) .2_{2} \cong$ $\mathrm{PGO}_{6}^{+}(3)$.

It is interesting to consider the asymptotic behavior of $s(G)$ and $u(G)$ for infinite sequences of simple groups $G$. In [27] it is proved that $s\left(A_{n}\right)$ tends to infinity if and only if the smallest prime divisor of $n$ tends to infinity. More generally, [27, Theorem 1.1] states that if $G_{i}$ is a sequence of simple groups such that $\left|G_{i}\right| \rightarrow \infty$, then $s\left(G_{i}\right) \rightarrow \infty$ if and only if there does not exist an infinite subsequence of the $G_{i}$ consisting either of odd-dimensional orthogonal groups over a field of fixed size or of alternating groups $A_{n_{i}}$ with each $n_{i}$ divisible by a fixed prime. In fact, if we exclude these exceptional cases, then the proof actually shows that $u\left(G_{i}\right) \rightarrow \infty$. (This observation is originally due to Guralnick and Kantor [23].) Here we extend the analysis to sequences of suitable almost simple groups with socle $\operatorname{PSL}_{n}(q)$.

THEOREM 4. Let $G_{i}=\left\langle S_{i}, g_{i}\right\rangle$ be a sequence of almost simple groups, where $S_{i}=\operatorname{PSL}_{n_{i}}\left(q_{i}\right)$ and $\left|G_{i}\right|$ tends to infinity. Then $u\left(G_{i}\right)$ is bounded if and only if there exists an infinite subsequence of the $G_{i}$ where $n_{i}$ is odd, $q_{i}$ is fixed, and each $g_{i}$ is either a graph automorphism or a graph-field automorphism involving an odd-order field automorphism.

Our final result concerns the minimal generation of almost simple groups. Recall that every finite simple group is 2-generated. More generally, if $G$ is almost simple with socle $G_{0}$, then a theorem of Dalla Volta and Lucchini [16, Theorem 1] states that

$$
d(G)=\max \left\{2, d\left(G / G_{0}\right)\right\} \leq 3 .
$$

As an easy corollary of Theorem 3 , we recover this result in the case $G_{0}=$ $\operatorname{PSL}_{n}(q)$.

COROLlary 5. Let $G$ be an almost simple group with socle $G_{0}=\operatorname{PSL}_{n}(q)$. Then

$$
d(G)=\max \left\{2, d\left(G / G_{0}\right)\right\} \leq 3
$$


Let $G=\left\langle\mathrm{PSL}_{n}(q), g\right\rangle$ be an almost simple group. Bounds on fixed point ratios play an essential role in the proof of Theorem 3 (from which Theorem 2 quickly follows). Recall that if $\Omega=G / H$ is a transitive $G$-set, then the fixed point ratio (fpr) of an element $x \in G$, which we denote by $\operatorname{fpr}(x, G / H)$, is the proportion of points in $\Omega$ fixed by $x$. Our approach relies on the following easy observation (see Theorem 2.3). Suppose that there exists $s \in \mathrm{PSL}_{n}(q)$ such that

$$
\sum_{H \in \mathcal{M}(g s)} \operatorname{fpr}(x, G / H)<1 / 2
$$

for all $x \in G$ of prime order, where $\mathcal{M}(g s)$ is the set of maximal subgroups of $G$ containing $g s$. Then the conclusion to Theorem 3 holds with $C=(g s)^{G}$. In almost all cases, we will show that there exists such an element $s$.

There are several steps in estimating the summation in (2). First, we need to choose $s$ in such a way that we can determine the subgroups in $\mathcal{M}(g s)$; the basic idea is to choose $s$ so that $g s$ is contained in very few maximal subgroups, and we use a combination of tools to do this. For example, we frequently apply the main theorem of [25] (and related results in [24, Section 2]) on subgroups containing elements of large prime orders (see Section 2.5), and we use the theory of Shintani descent in the case where $g$ is a field or graph-field automorphism (see Section 2.6). Next, we require upper bounds on fixed point ratios for elements of prime order in primitive actions of $G$ (see Section 2.4). Fortunately, such bounds have been widely studied in recent years (see, e.g., [9]-[12] and [23, Section 3]). Our aim is to obtain an explicit bound of the form

$$
\sum_{H \in \mathcal{M}(g s)} \operatorname{fpr}(x, G / H)<F(n, q)
$$

for some function $F$ with the property that $F(n, q)<1 / 2$ for all suitable values of $n$ and $q$. In addition, if $F(n, q)$ tends to 0 as $n$ or $q$ tends to infinity, then the conclusion to Theorem 4 also follows. For some small values on $n$ and $q$, we frequently require a more detailed analysis; in these cases it is often convenient to verify the desired bound directly, with the aid of MAGMA (see [4]; see Section 2.7 for further details).

In a forthcoming article, we extend our techniques and analysis to the other almost simple groups of Lie type. Combined with Guralnick's reduction theorem [22], and earlier work on groups with an alternating or sporadic 
socle, this will complete the proof of the Breuer-Guralnick-Kantor conjecture on 3/2-generated finite groups.

Our article has the following organization. In Section 2, we fix notation and present a number of results which will be required in the proofs of the main theorems. More precisely, in Section 2.2 we describe the probabilistic methods at the heart of our proof of Theorem 3-the main result here is Theorem 2.3. In Section 2.3, we give a brief overview of the subgroup structure of almost simple groups with socle $\mathrm{PSL}_{n}(q)$, based on Aschbacher's main theorem in [1]. Various bounds on fixed point ratios are presented in Section 2.4, and some useful results on primitive prime divisors are recalled in Section 2.5. The theory of Shintani descent plays an important role in our analysis - this is explained in Section 2.6. Finally, in Section 2.7 we discuss the role of MAGMA in the proofs of the main theorems.

The proofs of Theorems $2-4$ is given in Sections $3-6$. Here the analysis naturally splits into four cases, according to the various possibilities for $g$. In Section 3, we quickly handle the case where $g$ is a diagonal automorphism (this is essentially given in [7]). In Sections 4 and 5, we assume that $g$ is a field or graph-field automorphism, and we complete the proof in Section 6 , where we deal with graph automorphisms. Finally, the short proof of Corollary 5 is presented in Section 7.

\section{$\S 2$. Preliminaries}

\subsection{Notation and terminology}

We start by fixing some of the notation we use throughout this paper. Let $G$ be a finite group, and let $n$ be a positive integer. We write $G^{n}$ for the direct product of $n$ copies of $G, Z(G)$ for the center of $G$, and we write $|x|$ for the order of an element $x \in G$. The exact spread and exact uniform spread of $G$ are denoted by $s(G)$ and $u(G)$, respectively. The cyclic group of order $n$ is denoted by $Z_{n}$ (or just $n$ ), while $\mathbb{F}_{q}$ is the finite field of order $q$. For integers $a$ and $b,(a, b)$ denotes their highest common factor, and $\delta_{a, b}$ is the familiar Kronecker delta (so that $\delta_{a, b}=1$ if $a=b$; otherwise, $\delta_{a, b}=0$ ). We adopt the standard terminology and notation of [33] for finite classical groups and their subgroups. In particular, we write $\mathrm{GL}_{n}^{+}(q)=\mathrm{GL}_{n}(q)$ and $\mathrm{GL}_{n}^{-}(q)=\mathrm{GU}_{n}(q)$, and we extend this notation to the projective groups $\operatorname{PGL}_{n}^{\epsilon}(q)$ and $\operatorname{PSL}_{n}^{\epsilon}(q)$ in the obvious way. We will often represent an element of $(\mathrm{P}) \mathrm{GL}(V)$ as a matrix with respect to a fixed basis of $V$; it is convenient to write $\left[A_{1}, \ldots, A_{t}\right]$ 
to denote a block-diagonal matrix with blocks $A_{i}$. We will also write $J_{m}$ for a standard unipotent Jordan block of size $m$.

Let $G_{0}=\operatorname{PSL}_{n}(q)$, where $n \geq 2$ and $q=p^{f}$ for a prime $p$. By a theorem of Steinberg (see [46, Theorem 30]), every automorphism of $G_{0}$ is a product of the form $i d f g$, where

- $i$ is an inner automorphism (induced by conjugation in $G_{0}$ ),

- $d$ is a diagonal automorphism (induced by conjugation in $\mathrm{PGL}_{n}(q) \backslash G_{0}$ ),

- $f$ is a field automorphism (induced by an automorphism of $\mathbb{F}_{q}$ ), and

- $g$ is a graph automorphism (induced by the order 2 symmetry of the associated Dynkin diagram of type $A_{n-1}$, with $n \geq 3$ ).

As the terminology suggests, an inner-diagonal automorphism is the product of an inner and a diagonal automorphism. Naturally, we identify $G_{0}$ and $\operatorname{PGL}_{n}(q)$ with the subgroups of $\operatorname{Aut}\left(G_{0}\right)$ comprising the inner and innerdiagonal automorphisms of $G_{0}$, respectively. The full automorphism group has structure

$$
\operatorname{Aut}\left(G_{0}\right)=\left(G_{0} \rtimes Z_{(n, q-1)}\right) \rtimes\left(Z_{f} \times Z_{a}\right),
$$

where $a=2$ if $n \geq 3$; otherwise, $a=1$.

\subsection{Probabilistic methods}

Let $G$ be a finite group. For $x, y \in G$, we define

$$
\mathbb{P}(x, y)=1-\frac{\left|\left\{z \in y^{G} \mid G=\langle x, z\rangle\right\}\right|}{\left|y^{G}\right|}=\frac{\left|\left\{z \in y^{G} \mid G \neq\langle x, z\rangle\right\}\right|}{\left|y^{G}\right|},
$$

the probability that $x$ and a randomly chosen conjugate of $y$ do not generate $G$.

LEMMA 2.1. Suppose that there exist an element $y \in G$ and a positive integer $k$ such that $\mathbb{P}(x, y)<1 / k$ for all nontrivial $x \in G$. Then $u(G) \geq k$.

Proof. Let $\mathbb{P}(E)$ be the probability that an event $E$ occurs, and let $E^{c}$ denote the complementary event, so that $\mathbb{P}\left(E^{c}\right)=1-\mathbb{P}(E)$. Suppose that $x_{1}, \ldots, x_{k} \in G$ are nontrivial, and let $E_{i}$ be the event that $G=\left\langle x_{i}, z\right\rangle$, where $z$ is a randomly chosen $G$-conjugate of $y$. Clearly, it suffices to show that $\mathbb{P}(E)>0$, where $E=E_{1} \cap \cdots \cap E_{k}$. Now

$$
\mathbb{P}(E)=1-\mathbb{P}\left(E^{c}\right)=1-\mathbb{P}\left(E_{1}^{c} \cup \cdots \cup E_{k}^{c}\right) \geq 1-\sum_{i=1}^{k} \mathbb{P}\left(E_{i}^{c}\right),
$$

and $\mathbb{P}\left(E_{i}^{c}\right)=\mathbb{P}\left(x_{i}, y\right)<1 / k$, so $\mathbb{P}(E)>1-k(1 / k)=0$ as required. 
Consequently, we see that Theorem 3 implies Theorem 2 (modulo checking the four exceptions in (1)).

Clearly, we need to estimate $\mathbb{P}(x, y)$ in order to apply Lemma 2.1 effectively. To do this, we will use the bound provided in Lemma 2.2 below, but first we require some additional notation. For $y \in G$, let $\mathcal{M}(y)$ denote the set of maximal subgroups of $G$ containing $y$. Let $\Omega=G / H$ denote the set of (right) cosets of a subgroup $H$ in $G$, and let $\operatorname{fpr}(x, G / H)$ be the fixed point ratio of $x \in G$ with respect to the natural transitive action of $G$ on $G / H$, so $\operatorname{fpr}(x, G / H)$ is the proportion $\left|C_{\Omega}(x)\right| /|\Omega|$ of points in $\Omega$ that are fixed by $x$, where $C_{\Omega}(x)=\{\omega \in \Omega \mid \omega x=\omega\}$ is the set of fixed points of $x$ on $\Omega$. It is straightforward to show that

$$
\operatorname{fpr}(x, G / H)=\frac{\left|x^{G} \cap H\right|}{\left|x^{G}\right|} .
$$

Lemma 2.2. For any $x, y \in G$ we have

$$
\mathbb{P}(x, y) \leq \sum_{H \in \mathcal{M}(y)} \operatorname{fpr}(x, G / H) .
$$

Proof. Suppose that $z \in y^{G}$ and $G \neq\langle x, z\rangle$. Then $\left\langle x^{\prime}, y\right\rangle \leq H$ for some $H \in \mathcal{M}(y)$ and $x^{\prime} \in x^{G}$. The bound now follows since $\operatorname{fpr}(x, G / H)$ is the probability that a randomly chosen $G$-conjugate $x^{\prime}$ of $x$ has the property $\left\langle x^{\prime}, y\right\rangle \leq H$.

Clearly, we have

$$
\sum_{H \in \mathcal{M}(y)} \operatorname{fpr}(x, G / H) \leq \sum_{H \in \mathcal{M}\left(y^{i}\right)} \operatorname{fpr}\left(x^{j}, G / H\right)
$$

for all positive integers $i, j$. In particular, Theorem 3 follows immediately from the next result.

TheOREM 2.3. Let $G=\left\langle\operatorname{PSL}_{n}(q), g\right\rangle$ be an almost simple group, and assume that $G$ is not one of the groups listed in (1). Then there exists an element $s \in G_{0}$ such that

$$
\sum_{H \in \mathcal{M}(g s)} \operatorname{fpr}(x, G / H)<1 / 2
$$

for all $x \in G$ of prime order. 
Our strategy, therefore, is to find a suitable element $s \in G_{0}$ so that we can determine the maximal subgroups in $\mathcal{M}(g s)$, or at least the subgroups in the superset $\mathcal{M}\left((g s)^{i}\right)$ for some suitable positive integer $i$. The basic idea is to choose $s$ so that $g s$ is contained in very few maximal subgroups of $G$. Moreover, if we set $\alpha(x)=\sum_{H \in \mathcal{M}(g s)} \operatorname{fpr}(x, G / H)$, then in order to prove Theorem 4, it suffices to show that $\alpha(x) \rightarrow 0$ as $|G| \rightarrow \infty$ for all relevant $G$ and all $x \in G$ of prime order. In general, we do this by obtaining an explicit upper bound of the form $\alpha(x)<F(n, q)$ with the property that $F(n, q) \rightarrow 0$ as $n$ or $q$ tends to infinity.

Finally, we record a general result on the number of points in $\Omega$ fixed by an element $x \in G$.

LEMMA 2.4. Let $G$ be a finite transitive permutation group on a set $\Omega$ with point stabilizer $H$. Suppose that $x \in G$ and that $x^{G} \cap H$ is the union of $r$ distinct $H$-classes, with representatives $x_{1}, \ldots, x_{r}$. Then

$$
\left|C_{\Omega}(x)\right|=\left|C_{G}(x)\right| \sum_{i=1}^{r}\left|C_{H}\left(x_{i}\right)\right|^{-1}=\sum_{i=1}^{r}\left[C_{G}\left(x_{i}\right): C_{H}\left(x_{i}\right)\right]
$$

Proof. By (3) we have

$$
\left|C_{\Omega}(x)\right|=\frac{\left|x^{G} \cap H\right|}{\left|x^{G}\right|} \cdot[G: H]=\frac{\left|C_{G}(x)\right|}{|H|} \sum_{i}\left|x_{i}^{H}\right|=\left|C_{G}(x)\right| \sum_{i}\left|C_{H}\left(x_{i}\right)\right|^{-1} .
$$

The final equality holds since $\left|C_{G}(x)\right|=\left|C_{G}\left(x_{i}\right)\right|$ for all $i$.

Corollary 2.5. Let $G$ be a finite group, let $H$ be a self-normalizing subgroup of $G$, and let $x \in H$. Let $N$ be the number of distinct $G$-conjugates of $H$ containing $x$. Then $N=1$ if and only if $C_{G}(x)=C_{H}(x)$ and $x^{G} \cap H=$ $x^{H}$.

Proof. Suppose that $x^{G} \cap H$ is the union of $r$ distinct $H$-classes, with representatives $x_{1}, \ldots, x_{r}$. Since $N_{G}(H)=H$, Lemma 2.4 implies that

$$
N=\frac{\left|x^{G} \cap H\right|}{\left|x^{G}\right|} \cdot[G: H]=\sum_{i=1}^{r}\left[C_{G}\left(x_{i}\right): C_{H}\left(x_{i}\right)\right]
$$

and the result follows. 
Table 1: The $\mathcal{C}_{i}$ collections.

\begin{tabular}{|l|l|}
\hline $\mathcal{C}_{1}$ & Stabilizers of subspaces, or pairs of subspaces, of $V$ \\
\hline $\mathcal{C}_{2}$ & Stabilizers of decompositions $V=\bigoplus_{i=1}^{t} V_{i}$, where $\operatorname{dim} V_{i}=a$ \\
\hline $\mathcal{C}_{3}$ & Stabilizers of prime index extension fields of $\mathbb{F}_{q}$ \\
\hline $\mathcal{C}_{4}$ & Stabilizers of decompositions $V=V_{1} \otimes V_{2}$ \\
\hline $\mathcal{C}_{5}$ & Stabilizers of prime index subfields of $\mathbb{F}_{q}$ \\
\hline $\mathcal{C}_{6}$ & $\begin{array}{l}\text { Normalizers of symplectic-type } r \text {-groups in absolutely irreducible } \\
\text { representations }\end{array}$ \\
\hline $\mathcal{C}_{7}$ & Stabilizers of decompositions $V=\bigotimes_{i=1}^{t} V_{i}$, where $\operatorname{dim} V_{i}=a$ \\
\hline $\mathcal{C}_{8}$ & Stabilizers of nondegenerate forms on $V$ \\
\hline $\mathcal{C}_{9}$ & Almost simple irreducible subgroups of $G$ \\
\hline
\end{tabular}

\subsection{Subgroup structure}

Let $G$ be an almost simple group with socle $G_{0}=\operatorname{PSL}_{n}(q)$ and natural module $V$ over $\mathbb{F}_{q}$. The main theorem on the subgroup structure of finite classical groups is due to Aschbacher [1]. In [1], nine subgroup collections are defined, labeled $\mathcal{C}_{i}$ for $1 \leq i \leq 9$, and the main theorem states that if $H$ is a maximal subgroup of $G$ not containing $G_{0}$, then $H$ is contained in one of these collections. A rough description of the various $\mathcal{C}_{i}$ collections is given in Table 1.

We refer the reader to [33] (and, in particular, to [33, Table 3.5.A] for the case $G_{0}=\operatorname{PSL}_{n}(q)$ we are interested in here) for a detailed analysis of these various subgroup collections. Throughout this article, we adopt the standard notation and terminology of [33]. In particular, if $H$ is a maximal subgroup of $G$, then the type of $H$ provides a rough group-theoretic description of $H$. For example, if $n=6$ and if $H$ is the $G$-stabilizer of a direct sum decomposition $V=V_{1} \oplus V_{2} \oplus V_{3}$ with $\operatorname{dim} V_{i}=2$, then we say that $H$ is a $\mathcal{C}_{2}$-subgroup of type $\mathrm{GL}_{2}(q) \imath S_{3}$.

\subsection{Fixed point ratios}

Let $G=\left\langle G_{0}, g\right\rangle$ be an almost simple group with socle $G_{0}=\operatorname{PSL}_{n}(q)$, where $q=p^{f}$ for a prime $p$. Recall that in order to prove Theorem 2.3 we need to estimate the sum

$$
\sum_{H \in \mathcal{M}(g s)} \operatorname{fpr}(x, G / H)
$$


for certain elements $g s$ in the coset $g G_{0}$, where $x \in G$ has prime order. As previously described, Aschbacher's theorem (combined with the analysis in [33, Chapter 4]) provides us with detailed information on the possible subgroups in $\mathcal{M}(g s)$. Given a maximal subgroup $H \in \mathcal{M}(g s)$, our attention now turns to the corresponding fixed point ratio $\operatorname{fpr}(x, G / H)$, where $x \in G$ is an arbitrary element of prime order. To prove Theorem 2.3, we require good upper bounds on $\operatorname{fpr}(x, G / H)$.

The study of fixed point ratios dates back to the early days of group theory in the nineteenth century, and in recent years our understanding of fixed point ratios for almost simple primitive groups has advanced greatly. For example, Liebeck and Saxl [35] prove that if $n \geq 5$, then $\operatorname{fpr}(x, G / H) \leq 4 / 3 q$ for all $x \in G$ of prime order (this is a special case of [35, Theorem 1]), and there are examples (for arbitrary $n$ and $q$ ) where this upper bound is essentially sharp. For instance, if $H$ is the stabilizer of a 1-dimensional subspace of $V$, then $\operatorname{fpr}(x, G / H)$ is roughly $1 / q$ when $x$ is a transvection. However, we can establish much better bounds when $H$ is a so-called nonsubspace subgroup of $G$, which essentially means that $H$ acts irreducibly on $V$ (see [9, Definition 1] for the precise definition).

TheOREM 2.6. Let $G$ be a primitive permutation group with socle $G_{0}=$ $\mathrm{PSL}_{n}(q)$ and point stabilizer $H$, where $n \geq 3$ and where $H$ is a nonsubspace subgroup of $G$. Assume that $(n, q) \neq(4,2),(3,2)$. Then

$$
\operatorname{fpr}(x, G / H)<\left|x^{G}\right|^{-1 / 2+1 / n+\epsilon}
$$

for all $x \in G$ of prime order, where $\epsilon=1 / n$ if $H$ is of type $\operatorname{Sp}_{n}(q)$; otherwise, $\epsilon=0$.

Proof. This is a special case of [9, Theorem 1].

For the remaining subspace subgroups, we will use Theorem 2.7 below. Here the notation $P_{k}$ denotes a maximal parabolic subgroup of $G$ corresponding to the $G$-stabilizer of a $k$-dimensional subspace of $V$. In addition, $P_{k, n-k}$ denotes the $G$-stabilizer of a pair of subspaces $U \subseteq W$ of $V$, where $\operatorname{dim} U=k$ and $\operatorname{dim} W=n-k$. (Such a subgroup is maximal in $G$ whenever $G \not \mathrm{P} \mathrm{L}_{n}(q)$.)

THEOREM 2.7. Let $G$ be a primitive permutation group with socle $G_{0}=$ $\operatorname{PSL}_{n}(q)$ and point stabilizer $H$, where $n \geq 3$. Let $H$ be a subgroup of type 
Table 2: Some fixed point ratio bounds for $n \geq 5$.

\begin{tabular}{|l|l|l|}
\hline Type of $H$ & Condition & $g(n, q)$ \\
\hline $\mathrm{GL}_{1}(q) \times \mathrm{GL}_{n-1}(q)$ & $n$ odd & $q^{-2}$ \\
\hline $\mathrm{GL}_{n / 2}\left(q^{2}\right)$ & $n$ even & $2 q^{8-2 n}$ \\
\hline $\mathrm{Sp}_{n}(q)$ & $n$ even & $2 q^{2-n}$ \\
\hline$O_{n}^{\epsilon}(q)$ & $q$ odd & $2 q^{1-n}$ \\
\hline
\end{tabular}

$P_{k}, P_{k, n-k}$, or $\mathrm{GL}_{k}(q) \times \mathrm{GL}_{n-k}(q)$, where $k \leq n / 2$, and let $x \in G$ be an element of prime order. Then

$$
\operatorname{fpr}(x, G / H)< \begin{cases}\min \left\{1 / 2, q^{-1}+q^{1-n}\right\} & \text { if } k=1, \\ 2 q^{-k} & \text { otherwise. }\end{cases}
$$

Proof. This follows from [23, Proposition 3.1 and Lemma 3.12].

The next result provides sharper, or more explicit, bounds in some specific cases.

Proposition 2.8. Let $G$ be a primitive permutation group with socle $G_{0}=\operatorname{PSL}_{n}(q)$ and point stabilizer $H$, where $n \geq 5$ and where $H$ is one of the subgroups listed in Table 2. Then $\operatorname{fpr}(x, G / H)<g(n, q)$ for all $x \in G$ of prime order, where $g(n, q)$ is given in the final column of Table 2.

Proof. Let $x \in G$ be an element of prime order $r$. If $x \in H \cap \operatorname{PGL}(V)$, then $x$ is either semisimple (if $r \neq p$ ) or unipotent (if $r=p$ ); otherwise, either $x$ is a field automorphism (in which case, $r$ divides $\log _{p} q=f$ ), or $r=2$ and $x$ is either a graph-field automorphism (this requires $f$ to be even) or a graph automorphism. For $x \in H \cap \operatorname{PGL}(V)$, we define

$$
\nu(x)=\min \left\{\operatorname{dim}[\bar{V}, \lambda \hat{x}] \mid \lambda \in K^{*}\right\},
$$

where $\hat{x}$ is a preimage of $x$ in $\operatorname{GL}(V), \bar{V}=V \otimes K$ with $K=\overline{\mathbb{F}}_{q}$, and $[\bar{V}, \lambda \hat{x}]$ is the subspace $\langle v-v \lambda \hat{x} \mid v \in \bar{V}\rangle$. In particular, $\nu(x)$ is simply the codimension of the largest eigenspace of $\hat{x}$ on $\bar{V}$. Various bounds on $\left|x^{G}\right|$ in terms of $\nu(x)$ are presented in $\left[10\right.$, Section 3]. We will use the notation $\left[A_{1}, \ldots, A_{t}\right]$ to denote a block-diagonal matrix with blocks $A_{i}$, and we write $J_{m}$ for a standard unipotent Jordan block of size $m$.

First, assume that $H$ is a nonsubspace subgroup, so $H$ is of type $\mathrm{GL}_{n / 2}\left(q^{2}\right), \operatorname{Sp}_{n}(q)$, or $O_{n}^{\epsilon}(q)$. Here the desired result quickly follows from 
Theorem 2.6. For example, suppose that $H$ is of type $\operatorname{Sp}_{n}(q)$. For now, let us assume that $n \geq 8$. If $x \in H \backslash \operatorname{PGL}(V)$, then $\left|x^{G}\right|>(1 / 2) q^{\left(n^{2}-n-4\right) / 2}$ by $[10$, Corollary 3.49], and the bound in Theorem 2.6 is sufficient. Now assume that $x \in H \cap \operatorname{PGL}(V)$. If $\nu(x) \geq 2$, then $\left|x^{G}\right|>(1 / 2) q^{4 n-8}$ by [10, Corollary 3.38], and once again the result follows from Theorem 2.6. Finally, if $\nu(x)=1$, then $x=\left[J_{2}, I_{n-2}\right]$ is a transvection, and using (3) we calculate that $\operatorname{fpr}(x, G / H)<2 q^{2-n}$ since

$$
\left|x^{G} \cap H\right|=\frac{\left|\operatorname{Sp}_{n}(q)\right|}{\left|\operatorname{Sp}_{n-2}(q)\right| q^{2 n-1}}=q^{n}-1
$$

and

$$
\left|x^{G}\right|=\frac{\left|\mathrm{GL}_{n}(q)\right|}{\left|\mathrm{GL}_{n-2}(q)\right|\left|\mathrm{GL}_{1}(q)\right| q^{2 n-3}}=\frac{\left(q^{n-1}-1\right)\left(q^{n}-1\right)}{q-1} .
$$

If $n=6$, then we can analyze the various possibilities for $x$ in more detail, following the proof of [10, Proposition 8.1]. We leave the details to the reader. In the same way, we can deal with the other nonsubspace subgroups in Table 2.

For the remainder, let us assume that $n$ is odd and that $H$ is of type $\mathrm{GL}_{1}(q) \times \mathrm{GL}_{n-1}(q)$. Let $x \in H$ be an element of prime order $r$. There are several cases to consider, distinguished by the various possibilities for $r$.

First, assume that $x \in H \cap \operatorname{PGL}(V)$. Suppose that $r=2$ and that $p>2$. If $\nu(x)=1$, then

$$
\begin{aligned}
\left|x^{G} \cap H\right| & \leq 1+\frac{\left|\mathrm{GL}_{n-1}(q)\right|}{\left|\mathrm{GL}_{1}(q)\right|\left|\mathrm{GL}_{n-2}(q)\right|}=1+\frac{q^{n-2}\left(q^{n-1}-1\right)}{q-1}, \\
\left|x^{G}\right| & =\frac{\left|\mathrm{GL}_{n}(q)\right|}{\left|\mathrm{GL}_{1}(q)\right|\left|\mathrm{GL}_{n-1}(q)\right|}=\frac{q^{n-1}\left(q^{n}-1\right)}{q-1},
\end{aligned}
$$

and the result follows. Similarly, if $\nu(x)=s \geq 2$, then the bounds

$$
\begin{aligned}
\left|x^{G} \cap H\right| & \leq \frac{\left|\mathrm{GL}_{n-1}(q)\right|}{\left|\mathrm{GL}_{s}(q)\right|\left|\mathrm{GL}_{n-s-1}(q)\right|}+\frac{\left|\mathrm{GL}_{n-1}(q)\right|}{\left|\mathrm{GL}_{s-1}(q)\right|\left|\mathrm{GL}_{n-s}(q)\right|} \\
& <2 q^{\gamma}\left(q^{-2(n-s)}+q^{-2 s}\right)
\end{aligned}
$$

and $\left|x^{G}\right|>(1 / 4) q^{\gamma}$, where $\gamma=2 n s-2 s^{2}$, are good enough.

Next, suppose that $r=p$. Let $a_{i}$ denote the number of Jordan blocks of size $i$ in the Jordan form of $\hat{x}$ on $\bar{V}$. Then $a_{i}=0$ for all $i>p$, and $a_{1} \geq 1$ (since $r=p$ and $x \in H$ ). Let $t$ denote the number of nonzero $a_{i}$ terms, and 
note that $t \geq 2$. If $a_{i}=0$ for all $i>2$, say, $x=\left[J_{2}^{s}, J_{1}^{n-2 s}\right]$ for some $s \geq 1$, then it is easy to see that

$$
\operatorname{fpr}(x, G / H) \leq\left(q^{n-2 s}-1\right) /\left(q^{n}-1\right),
$$

and the result follows. In the remaining cases (with $r=p$ ), we may assume that $p>2$. By applying [10, Lemma 3.18], we deduce that

$$
\left|x^{G} \cap H\right|<2^{t-1} q^{\gamma-2 n+2 \sum_{i} a_{i}}
$$

and $\left|x^{G}\right|>(1 / 2) q^{\gamma}$, where $\gamma=n^{2}-2 \sum_{i<j} i a_{i} a_{j}-\sum_{i} i a_{i}^{2}$, whence

$$
\operatorname{fpr}(x, G / H)<2^{t} q^{-2 n+2 \sum_{i} a_{i}} .
$$

If $t=2$, then we may assume that $a_{i}>0$ for some $i>2$; hence, $\sum_{i} a_{i} \leq n-2$, and thus $\operatorname{fpr}(x, G / H)<2^{2} q^{-4}<q^{-2}$ as required. Similarly if $t \geq 3$, then $\sum_{i} a_{i} \leq n-t(t-1) / 2$, and this yields

$$
\operatorname{fpr}(x, G / H)<2^{t} q^{-2 n+2(n-t(t-1) / 2)}=2^{t} q^{-t(t-1)}<q^{2 t-t^{2}} \leq q^{-3} .
$$

Now assume that $r \neq p$ and that $r>2$. Let $i \geq 1$ be minimal such that $r$ divides $q^{i}-1$. Then $C_{G}(x)$ is of type

$$
\mathrm{GL}_{l}(q) \times \prod_{j=1}^{d} \mathrm{GL}_{a_{j}}\left(q^{i}\right)
$$

for some $d \geq 1$, where $n=l+i \sum_{j} a_{j}$ and $a_{j} \geq 1$ for all $j$. Set $\gamma=n^{2}-l^{2}-$ $i \sum_{j} a_{j}^{2}$. First, assume that $i \geq 2$, so $l \geq 1$ (since $x \in H$ ). Then $\left|x^{G} \cap H\right|<$ $2^{d} q^{\gamma-2(n-l)}$ and $\left|x^{G}\right|>(1 / 2) q^{\gamma}$, so

$$
\operatorname{fpr}(x, G / H)<2^{d+1} q^{-2(n-l)} \leq q^{1-3 d} \leq q^{-2},
$$

since $n-l \geq 2 d$ and $d \geq 1$. Now assume that $i=1$ (so $q \geq 4$ ). If $d=1$, then we may argue as in the case $r=2$ with $p>2$, so let us assume that $d \geq 2$. Without loss, we may assume that $l \geq a_{1}$. Then $\left|x^{G}\right|>(1 / 2) q^{\gamma-1}$ and

$$
\left|x^{G} \cap H\right| \leq(d+1) \cdot \frac{\left|\mathrm{GL}_{n-1}(q)\right|}{\left|\mathrm{GL}_{l-1}(q)\right| \prod_{j}\left|\mathrm{GL}_{a_{j}}\left(q^{i}\right)\right|}<2^{d}(d+1) q^{\gamma-2(n-l)} .
$$

Therefore,

$$
\operatorname{fpr}(x, G / H)<2^{d+1}(d+1) q^{1-2(n-l)}<q^{d+1+1-2 d}=q^{2-d},
$$


and we reduce to the case $d \leq 3$. If $(i, d)=(1,3)$, then $q \geq 11$ (since $r \geq 5$ ), and the above bounds give $\operatorname{fpr}(x, G / H)<64 q^{-5}<q^{-3}$. Finally, suppose that $(i, d)=(1,2)$. If $n-l>2$, then $n-l \geq 3$, and thus $\operatorname{fpr}(x, G / H)<24 q^{-5}<$ $q^{-2}$ since $q \geq 4$. Otherwise, if $n-l=2$, then $\left|x^{G} \cap H\right|<4 q^{4 n-10}+4 q^{2 n-4}$, $\left|x^{G}\right|>\frac{1}{2} q^{4 n-6}$, and the result follows.

Finally, suppose that $x \in H \backslash \operatorname{PGL}(V)$. First, assume that $x$ is an involutory graph automorphism, so $\left|x^{G}\right|>(1 / 2) q^{\left(n^{2}+n-4\right) / 2}$ (see [10, Table 3.11]). Now $x$ induces a graph automorphism on the factor of $H$ of type $\operatorname{GL}_{n-1}(q)$, so by considering the centralizer types listed in [10, Table 3.10], we deduce that if $p=2$, then

$$
\left|x^{G} \cap H\right| \leq \frac{\left|\mathrm{GL}_{n-1}(q)\right|}{\left|\operatorname{Sp}_{n-1}(q)\right|}+\frac{\left|\mathrm{GL}_{n-1}(q)\right|}{\left|C_{\mathrm{Sp}_{n-1}(q)}(t)\right|}<q^{(1 / 2)\left(n^{2}-3 n+2\right)}+q^{(1 / 2) n(n-1)},
$$

where $t \in \operatorname{Sp}_{n-1}(q)$ is a transvection, while

$$
\begin{aligned}
\left|x^{G} \cap H\right| & \leq \frac{\left|\mathrm{GL}_{n-1}(q)\right|}{\left|\mathrm{Sp}_{n-1}(q)\right|}+\frac{\left|\mathrm{GL}_{n-1}(q)\right|}{\left|\mathrm{SO}_{n-1}^{+}(q)\right|}+\frac{\left|\mathrm{GL}_{n-1}(q)\right|}{\left|\mathrm{SO}_{n-1}^{-}(q)\right|} \\
& <q^{(1 / 2)\left(n^{2}-3 n+2\right)}+2 q^{(1 / 2) n(n-1)}
\end{aligned}
$$

if $p>2$. These bounds are sufficient unless $n=5$ and $q \leq 3$; here the desired result is quickly obtained through direct calculation.

If $x$ is an involutory field or graph-field automorphism, then $q=q_{0}^{2}$ and the bounds

$$
\begin{aligned}
\left|x^{G} \cap H\right| & \leq \frac{\left|\mathrm{GL}_{1}(q)\right|}{\left|\mathrm{GL}_{1}\left(q_{0}\right)\right|} \cdot \frac{\left|\mathrm{GL}_{n-1}(q)\right|}{\left|\mathrm{GL}_{n-1}\left(q_{0}\right)\right|}<4 q^{(1 / 2)\left(n^{2}-2 n+2\right)}, \\
\left|x^{G}\right| & >\frac{1}{2}(n, q-1)^{-1} q^{(1 / 2)\left(n^{2}-1\right)}
\end{aligned}
$$

are sufficient. Finally, if $x$ is a field automorphism of odd prime order $r$, then $q=q_{0}^{r}$ and

$$
\left|x^{G} \cap H\right|<4 q^{\left(1+(n-1)^{2}\right)(1-1 / r)}, \quad\left|x^{G}\right|>\frac{1}{2} q^{\left(n^{2}-1\right)(1-1 / r)-1} ;
$$

hence,

$$
\operatorname{fpr}(x, G / H)<8 q^{(3-2 n)(1-1 / r)+1} \leq 8 q^{3-(4 / 3) n} \leq q^{4-(4 / 3) n} .
$$

The result follows. 
Corollary 2.9. Suppose that $n \geq 3$, that $H$ is a nonsubspace subgroup, and that $(n, q) \neq(4,2),(3,2)$. Also assume that $n \geq 6$ if $H$ is of type $\operatorname{Sp}_{n}(q)$. Then $\operatorname{fpr}(x, G / H)<f(n, q)$ for all $x \in G$ of prime order, where

$$
f(n, q)=\left(\frac{\left(q^{n-1}-1\right)\left(q^{n}-1\right)}{q-1}\right)^{-(1 / 2)+(1 / n)} .
$$

Proof. If $H$ is not of type $\operatorname{Sp}_{n}(q)$, then this follows immediately from Theorem 2.6 since

$$
\left|x^{G}\right| \geq \frac{\left|\mathrm{GL}_{n}(q)\right|}{\left|\mathrm{GL}_{n-2}(q)\right|\left|\mathrm{GL}_{1}(q)\right| q^{2 n-3}}=\frac{\left(q^{n-1}-1\right)\left(q^{n}-1\right)}{q-1}
$$

for all $x \in G$ of prime order (minimal if $x$ is a transvection). Now assume that $H$ is of type $\operatorname{Sp}_{n}(q)$. If $x \in H \cap \operatorname{PGL}(V)$ and $\nu(x)=1$, then $\operatorname{fpr}(x, G / H)=$ $(q-1) /\left(q^{n-1}-1\right)$ (see the proof of Proposition 2.8), which is less than $f(n, q)$. If $n \geq 8$ and $x \in H$ is not a transvection, then $\left|x^{G}\right|>(1 / 2) q^{4 n-8}$ (see [10, Corollaries 3.38 and 3.49]), and the bound in Theorem 2.6 is good enough. Finally, the case $n=6$ can be dealt with directly, by considering each possibility for $x$ in turn. The reader can check the details.

More accurate bounds when $n=3$ or 4 are given in Lemmas 2.10 and 2.11 below.

Lemma 2.10. Suppose that $n=3$ and that $x \in G$ has prime order.

(i) If $H$ is of type $O_{3}(q), \mathrm{GL}_{1}(q) 2 S_{3}, \mathrm{GL}_{1}\left(q^{3}\right)$, or $\mathrm{GL}_{3}\left(q_{1}\right)$, where $q=q_{1}^{r}$ for an odd prime $r$, then $\operatorname{fpr}(x, G / H) \leq\left(q^{2}+q+1\right)^{-1}$.

(ii) If $H$ is of type $\operatorname{GL}_{3}^{\epsilon}\left(q^{1 / 2}\right)$, then $\operatorname{fpr}(x, G / H) \leq(3, q-1) q^{-1 / 2}(q+1)^{-1}$.

Proof. This is a straightforward calculation. For example, suppose that $H$ is of type $O_{3}(q)$, in which case $q$ is odd. Set $d=(3, q-1)$, and let $x \in H$ be an element of prime order $r$. Suppose that $x \in H \cap \operatorname{PGL}(V)$. If $r=p$, then $x$ is conjugate to $\left[J_{3}\right]$, so $\left|x^{G} \cap H\right| \leq q^{2}-1,\left|x^{G}\right| \geq \frac{1}{d} q\left(q^{2}-1\right)\left(q^{3}-1\right)$, and the result follows. Similarly, if $r=2$, then $x$ is conjugate to $\left[-I_{2}, I_{1}\right]$, and we calculate that $\operatorname{fpr}(x, G / H) \leq\left(q^{2}+q+1\right)^{-1}$ since

$$
\begin{aligned}
\left|x^{G} \cap H\right| & \leq i_{2}\left(\mathrm{SO}_{3}(q)\right)=\frac{\left|\mathrm{SO}_{3}(q)\right|}{2\left|\mathrm{SO}_{2}^{+}(q)\right|}+\frac{\left|\mathrm{SO}_{3}(q)\right|}{2\left|\mathrm{SO}_{2}^{-}(q)\right|}=q^{2}, \\
\left|x^{G}\right| & =q^{2}\left(q^{2}+q+1\right)
\end{aligned}
$$

where $i_{2}\left(\mathrm{SO}_{3}(q)\right)$ denotes the number of involutions in $\mathrm{SO}_{3}(q)$. Next, suppose that $r \neq p$ and that $r$ is odd. Let $i \geq 1$ be minimal such that $r$ divides 
$q^{i}-1$, so $i=1$ or 2 . If $i=2$, then $\left|x^{G} \cap H\right|=q(q-1)$ and $\left|x^{G}\right|=q^{3}\left(q^{3}-1\right)$; otherwise, $\left|x^{G} \cap H\right|=q(q+1)$ and $\left|x^{G}\right| \geq \frac{1}{d} q^{3}(q+1)\left(q^{2}+q+1\right)$. In both cases the desired bound holds. Finally, suppose that $x \in H \backslash \operatorname{PGL}(V)$. If $r$ is odd, then $q=q_{0}^{r}$ and $x$ is a field automorphism; here the bounds $\left|x^{G} \cap H\right| \leq\left[\mathrm{SO}_{3}(q): \mathrm{SO}_{3}\left(q_{0}\right)\right]$ and $\left|x^{G}\right| \geq \frac{1}{d}\left[\operatorname{PGL}_{3}(q): \operatorname{PGL}_{3}\left(q_{0}\right)\right]$ are sufficient. Now assume that $r=2$. If $x$ is a field or graph-field automorphism, then $q=q_{0}^{2}$ and

$$
\begin{aligned}
\left|x^{G} \cap H\right| & \leq \frac{\left|\mathrm{SO}_{3}(q)\right|}{\left|\mathrm{SO}_{3}\left(q^{1 / 2}\right)\right|}=q^{1 / 2}(q+1), \\
\left|x^{G}\right| & \geq \frac{1}{d} \frac{\left|\mathrm{PGL}_{3}(q)\right|}{\left|\mathrm{PGU}_{3}\left(q^{1 / 2}\right)\right|}=\frac{1}{d} q^{3 / 2}(q+1)\left(q^{3 / 2}-1\right) ;
\end{aligned}
$$

otherwise, $x$ is a graph automorphism and we have

$$
\left|x^{G} \cap H\right| \leq i_{2}\left(\mathrm{SO}_{3}(q)\right)+1=q^{2}+1, \quad\left|x^{G}\right| \geq \frac{1}{d} q^{2}\left(q^{3}-1\right) .
$$

It is easy to check that these bounds are sufficient.

The other cases are very similar, and we leave the details to the reader. Note that if $H$ is of type $\mathrm{GL}_{3}\left(q^{1 / 2}\right)$ and $x \in H$ is an involutory graph-field automorphism, or if $H$ is of type $\mathrm{GU}_{3}\left(q^{1 / 2}\right)$ and $x \in H$ is an involutory field automorphism, then

$$
\begin{aligned}
\left|x^{G} \cap H\right| & \leq \frac{\left|\operatorname{PGL}_{3}^{\epsilon}\left(q^{1 / 2}\right)\right|}{\left|\operatorname{SO}_{3}\left(q^{1 / 2}\right)\right|}=q\left(q^{3 / 2}-\epsilon\right), \\
\left|x^{G}\right| & \geq \frac{1}{d} \frac{\left|\mathrm{PGL}_{3}(q)\right|}{\left|\mathrm{PGL}_{3}^{\epsilon}\left(q^{1 / 2}\right)\right|}=\frac{1}{d} q^{3 / 2}(q+1)\left(q^{3 / 2}-\epsilon\right),
\end{aligned}
$$

whence $\operatorname{fpr}(x, G / H) \leq d q^{-1 / 2}(q+1)^{-1}$ as claimed.

Similarly, we compute the following bounds when $n=4$. We omit the proof.

Lemma 2.11. Suppose that $n=4$. Let $H_{1}, H_{2}$, and $H_{3}$ be maximal subgroups of $G$ of type $\mathrm{GL}_{2}\left(q^{2}\right), \mathrm{Sp}_{4}(q)$, and $O_{4}^{-}(q)$, respectively. Then $\operatorname{fpr}\left(x, G / H_{i}\right) \leq f_{i}(q)$ for all $x \in G$ of prime order, where

$$
f_{1}(q)=\frac{d_{1}\left(q^{3}+2 q+1\right)}{q^{2}\left(q^{3}-1\right)}, \quad f_{2}(q)=\frac{q^{2}}{d_{2}\left(q^{3}-1\right)}, \quad f_{3}(q)=\frac{4 d_{2}}{q^{3}-1},
$$

with $d_{1}=(4, q-1)$ and $d_{2}=(2, q-1)$. 


\subsection{Primitive prime divisors}

Let $q=p^{a}$ be a prime power, and let $r$ be a prime dividing $q^{e}-1$. We say that $r$ is a primitive prime divisor ( $p$ pd for short) of $q^{e}-1$ if $r$ does not divide $q^{i}-1$ for all $1 \leq i<e$. In [48], Zsigmondy states that if $e \geq 3$, then either $q^{e}-1$ has a primitive prime divisor or $(q, e)=(2,6)$. Primitive prime divisors also exist when $e=2$, provided that $q$ is not a Mersenne prime. Note that if $r$ is a primitive prime divisor of $q^{e}-1$, then $r \equiv 1(\bmod e)$.

Let $G$ be an almost simple group with socle $G_{0}=\operatorname{PSL}_{n}(q)$, and let $r$ be a primitive prime divisor of $q^{e}-1$, where $n / 2<e \leq n$. In [25], the subgroups of $\mathrm{PGL}_{n}(q)$ containing an element of order $r$ are determined. As described in [25, Examples 2.1-2.9], it turns out that such a subgroup belongs to one of nine specific subgroup collections. Guralnick and Malle [24] prove the following useful corollary.

THEOREM 2.12. Let $G$ be an almost simple group with socle $G_{0}=\operatorname{PSL}_{n}(q)$ and natural module $V$, where $n \geq 3$. Let $r$ be either a primitive prime divisor of $q^{e}-1$, where $e>n / 2$ and $r>2 e+1$, or a product of two (possibly equal) primitive prime divisors of $q^{e}-1$. Suppose that $H$ is a maximal subgroup of $G$ such that $H \cap \mathrm{PGL}(V)$ acts irreducibly on $V$ and contains an element of order $r$. Then one of the following holds:

(i) $\quad H$ is of type $\mathrm{GU}_{n}\left(q^{1 / 2}\right), \operatorname{Sp}_{n}(q)$, or $O_{n}^{\epsilon}(q)$;

(ii) $H$ is of type $\mathrm{GL}_{n / k}\left(q^{k}\right)$, where $k$ is a prime dividing $(n, e)$;

(iii) $H$ is of type $\mathrm{GL}_{n}\left(q_{0}\right)$, where $q=q_{0}^{k}$ for some prime $k$.

Proof. This follows immediately from [24, Theorem 2.2].

Recall that our basic strategy for proving Theorem 2.3 is to find an element $s \in G_{0}$ such that $g s$ is contained in very few maximal subgroups of $G=\left\langle G_{0}, g\right\rangle$. If we can choose $s$ so that some power of $g s$ has order $r$, where $r$ is a primitive prime divisor of $q^{e}-1$ with $e>n / 2$, then we can use the aforementioned results in [24] and [25] to restrict significantly the possible subgroups in $\mathcal{M}(g s)$.

\subsection{Shintani descent}

Let $G_{0}$ be a simple group of Lie type over $\mathbb{F}_{q}$, and set $G=\left\langle G_{0}, g\right\rangle$ for some $g \in \operatorname{Aut}\left(G_{0}\right)$. In order to study the uniform spread of $G$, we need to consider conjugacy classes in the coset $g G_{0}$; as previously stated, our aim is to identify a class $(g s)^{G}$ such that $g s$ is contained in very few maximal subgroups of $G$. In the cases where $g$ is a field or graph-field automorphism, 
a key tool to do this is the theory of Shintani descent, which we outline below (following Kawanaka [32, Section 2]).

First, let us set up the notation we will use for the remainder of this section. Let $X$ be a connected linear algebraic group over an algebraically closed field, and let $\sigma: X \rightarrow X$ be a Frobenius morphism, so $\sigma$ is a bijective endomorphism of algebraic groups with finite fixed point subgroup $X_{\sigma}=$ $\left\{x \in X \mid x^{\sigma}=x\right\}$. Let $e$ be a positive integer, set $G=X_{\sigma^{e}}$, and set $H=X_{\sigma} \leq$ $G$. Note that $G$ is $\sigma$-stable, so the restriction $\sigma: G \rightarrow G$ is an automorphism. Let $A=\left\langle\sigma^{\prime}\right\rangle$ be a cyclic group of order $e$, and let $\phi: A \rightarrow \operatorname{Aut}(G)$ be the homomorphism such that $\phi\left(\sigma^{\prime}\right)=\sigma$. In the following we will abuse notation by writing $\sigma$ for $\sigma^{\prime}$. Let $A G=A \ltimes G$ be the corresponding semidirect product with multiplication

$$
\left(\sigma^{i}, s\right)\left(\sigma^{j}, t\right)=\left(\sigma^{i+j}, s^{\sigma^{j}} t\right)
$$

Let $\sigma s$ be an element in the coset $\sigma G$ in $A G$. Then $(\sigma s)^{2}=\sigma^{2} s^{\sigma} s$, and using the fact that $A$ has order $e$, we quickly deduce that

$$
(\sigma s)^{e}=s^{\sigma^{e-1}} s^{\sigma^{e-2}} \cdots s^{\sigma} s \in G
$$

By the Lang-Steinberg theorem (see [21, Theorem 2.1.1]), there exists $a \in X$ such that

$$
s=a^{-\sigma} a
$$

Using the expression for $(\sigma s)^{e}$ in (6), it is easy to check that $a(\sigma s)^{e} a^{-1}$ is fixed by $\sigma$, so $a(\sigma s)^{e} a^{-1} \in X_{\sigma}=H$. This observation allows us to define a map $f$ from the set of $A G$-classes in the coset $\sigma G$ to the set of $H$-classes in $H$ by

$$
f:(\sigma s)^{A G} \mapsto\left(a(\sigma s)^{e} a^{-1}\right)^{H},
$$

which we call the Shintani map of $G$ corresponding to $\sigma$. We will frequently abuse notation by writing $f(\sigma s)$ for an arbitrary representative of the $H$ class of $a(\sigma s)^{e} a^{-1}$. In addition, to avoid any possible ambiguity, we will sometimes write $f_{G}$, rather than $f$, for the above map.

We must check that $f$ is well defined. First, note that the element $a \in X$ given in (7) is not unique in general. However, if $b \in X$ also satisfies $s=b^{-\sigma} b$, then $a b^{-1} \in X_{\sigma}=H$, and thus the elements $a(\sigma s)^{e} a^{-1}$ and $b(\sigma s)^{e} b^{-1}$ are $H$-conjugate, so $f$ is independent of the choice of $a$ in (7). We also need to show that $f$ is independent of the choice of $A G$-class representative. To see 
this, suppose that $\sigma t \in \sigma G$ is $A G$-conjugate to $\sigma s$, say, $\sigma t=\left(\sigma^{i} w\right)^{-1} \sigma s\left(\sigma^{i} w\right)$ for some $w \in G$ and integer $i \geq 0$. Now

$$
\sigma t=(\sigma t)^{i}\left(\sigma^{i} w\right)^{-1} \sigma s\left(\sigma^{i} w\right)(\sigma t)^{-i}
$$

and $\left(\sigma^{i} w\right)(\sigma t)^{-i} \in G$, so $\sigma s$ and $\sigma t$ are in fact $G$-conjugate. (Consequently, $f$ is a map from the set of $G$-classes in the coset $\sigma G$ to the set of $H$ classes in $H$.) Therefore, there exists $z \in G$ such that $\sigma t=z^{-1} \sigma s z$; hence, $\sigma t=\sigma z^{-\sigma} s z$ and $t=z^{-\sigma} s z$. Since $s=a^{-\sigma} a$, we have $t=(a z)^{-\sigma} a z$, and thus $f(\sigma t)=a z(\sigma t)^{e} z^{-1} a^{-1}$. But $(\sigma t)^{e}=z^{-1}(\sigma s)^{e} z$ by assumption; hence, $f(\sigma t)=a(\sigma t)^{e} a^{-1}=f(\sigma s)$ and $f$ is well defined.

The next lemma is a key result (see [32, Lemma 2.2]).

LEMMA 2.13. With the notation above, the following hold.

(i) We have

$$
C_{G}(\sigma s)=a^{-1} C_{H}(f(\sigma s)) a=C_{a^{-1} H a}\left((\sigma s)^{e}\right) .
$$

In particular, $\left|C_{G}(\sigma s)\right|=\left|C_{H}(f(\sigma s))\right|$ for all $s \in G$.

(ii) The Shintani map $f$ is a bijection.

Proof. First, consider (i). If $g \in C_{G}(\sigma s)$, then $s g=g^{\sigma} s$, and clearly we have $a g a^{-1} \in C_{X}(f(\sigma s))$. Further, since $a^{\sigma}=a s^{-1}$ (see $(7)$ ), we see that $a g a^{-1} \in X_{\sigma}$, and thus $a g a^{-1} \in C_{H}(f(\sigma s))$. Conversely, suppose that $h \in$ $C_{H}(f(\sigma s))$. Then $a^{-1} h a$ centralizes $(\sigma s)^{e}$, and using (6) we deduce that $(\sigma s)^{e}=a^{-\sigma^{e}} a$. Therefore,

$$
\left(a^{-1} h a\right)^{\sigma^{e}}=a^{-\sigma^{e}} h a^{\sigma^{e}}=(\sigma s)^{e} a^{-1} h a(\sigma s)^{-e}=a^{-1} h a,
$$

and thus $a^{-1} h a \in X_{\sigma^{e}}=G$. Further, it is straightforward to check that $a^{-1} h a$ centralizes $\sigma s$, whence $a^{-1} h a \in C_{G}(\sigma s)$. This proves (i).

Now let us turn to (ii). First, we claim that

$$
\left|(\sigma s)^{A G}\right|=\left|f(\sigma s)^{H}\right| \cdot[G: H] .
$$

This follows easily from (i) since we have already observed that $(\sigma s)^{A G}=$ $(\sigma s)^{G}$ (see $\left.(8)\right)$.

Let $y \in H$. By the Lang-Steinberg theorem (applied to $X$ and $\sigma^{e}$ ), there exists $b \in X$ such that $y=b b^{-\sigma^{e}}$. Since $\sigma$ fixes $y$, it follows that $\sigma^{e}$ fixes $b^{-\sigma} b$, whence $b^{-\sigma} b \in G$. By definition, $f$ maps the $A G$-class of $\sigma b^{-\sigma} b$ to the $H$-class of $b\left(\sigma b^{-\sigma} b\right)^{e} b^{-1}$, and using the expression in (6), we calculate that

$$
b\left(\sigma b^{-\sigma} b\right)^{e} b^{-1}=b b^{-\sigma^{e}} b b^{-1}=y .
$$

This proves that the Shintani map $f$ is surjective. 
Finally, let $\left\{c_{1}, \ldots, c_{t}\right\}$ be the set of $H$-classes in $H$. Since $f$ is surjective, there exist $A G$-classes $C_{i}$ in $\sigma G$ such that $f\left(C_{i}\right)=c_{i}$ for all $i$. By (9) we have $\left|C_{i}\right|=\left|c_{i}\right| \cdot[G: H]$, so

$$
\sum_{i=1}^{t}\left|C_{i}\right|=[G: H] \sum_{i=1}^{t}\left|c_{i}\right|=|G|=|\sigma G|,
$$

and thus $\left\{C_{1}, \ldots, C_{t}\right\}$ is the complete set of $A G$-classes in $\sigma G$. We conclude that $f$ is a bijection.

THEOREM 2.14. With the notation above, let $Y$ be a closed connected $\sigma$ stable subgroup of $X$, let $K=Y_{\sigma^{e}}$, and let $L=Y_{\sigma}$. Let $\Omega=G / K$, and let $\Delta=H / L$. Then

$$
\left|C_{\Omega}(\sigma s)\right|=\left|C_{\Delta}(f(\sigma s))\right|
$$

for all $s \in G$.

Proof. Since $K$ is $\sigma$-stable, we may form the semidirect product $A K$. In addition, we obtain a well-defined action of $A G$ on $\Omega$ via $(K g)^{\sigma}=K g^{\sigma}$. Also note that since $Y$ is $\sigma$-stable and connected, the Shintani map $f=f_{G}$ on $G$ naturally induces a Shintani map $f_{K}$ from the set of $A K$-classes in the coset $\sigma K$ to the set of $L$-classes in $L$.

First, assume that $\left|C_{\Omega}(\sigma s)\right|=0$. If $(\sigma s)^{G} \cap \sigma K$ is nonempty, then $g^{-\sigma} s g \in$ $K$ for some $g \in G$, and thus $K g^{-1} \in C_{\Omega}(\sigma s)$, a contradiction. Therefore, $(\sigma s)^{G} \cap \sigma K$ is empty. We claim that $f_{G}(\sigma s)^{H} \cap L$ is also empty. To see this, suppose that there exists $h \in H$ such that $f_{G}(\sigma s)^{h} \in L$. By the Shintani correspondence given by $f_{K}$, there exists $k \in K$ such that $f_{K}(\sigma k)=f_{G}(\sigma s)^{h}$, where $f_{K}(\sigma k)=a(\sigma k)^{e} a^{-1}$ for some $a \in Y$ with $a^{-\sigma} a=k$. Since $a$ is also in $X$, we may assume that $f_{G}(\sigma k)=a(\sigma k)^{e} a^{-1}$, so $f_{G}(\sigma k)=f_{G}(\sigma s)^{h}$, and thus the Shintani correspondence given by $f_{G}$ implies that $\sigma k$ and $\sigma s$ are $G$ conjugate. This is a contradiction since $(\sigma s)^{G} \cap \sigma K$ is empty. This justifies the claim, and we conclude that $\left|C_{\Delta}(f(\sigma s))\right|=0$, as required.

Now suppose that $\left|C_{\Omega}(\sigma s)\right| \geq 1$, say, $K g \in C_{\Omega}(\sigma s)$. Then $g^{\sigma} s g^{-1} \in K$ and

$$
(\sigma s)^{g^{-1}}=\sigma g^{\sigma} s g^{-1} \in(\sigma s)^{G} \cap \sigma K
$$

is nonempty. Replacing $\sigma s$ by a suitable $G$-conjugate if necessary, we may assume that $\sigma s \in \sigma K$; that is, $s \in K$. Let $\sigma s_{1}, \ldots, \sigma s_{r}$ represent the distinct $A K$-classes in $(\sigma s)^{G} \cap \sigma K$. By considering the Shintani map $f_{K}$, and by applying Lemma 2.13(i), we deduce that

$$
\left|C_{K}\left(\sigma s_{i}\right)\right|=\left|C_{L}\left(f_{K}\left(\sigma s_{i}\right)\right)\right|=\left|C_{L}\left(f_{G}\left(\sigma s_{i}\right)\right)\right|
$$


for all $i$. (Here we may choose $f_{G}\left(\sigma s_{i}\right)=f_{K}\left(\sigma s_{i}\right) \in L$ to represent each of the relevant $H$-classes, so we can write $f$ for both $f_{G}$ and $f_{K}$, when convenient.)

Evidently, $A G$ acts transitively on $\Omega$ with point stabilizer $A K$, and we note that the $\sigma s_{i}$ represent the distinct $A K$-classes in $(\sigma s)^{A G} \cap A K$ since $(\sigma s)^{A G}=(\sigma s)^{G}$ and $\left(\sigma s_{i}\right)^{A K}=\left(\sigma s_{i}\right)^{K}$ for all $i$. Therefore, Lemma 2.4 yields

$$
\left|C_{\Omega}(\sigma s)\right|=\sum_{i=1}^{r}\left[C_{A G}\left(\sigma s_{i}\right): C_{A K}\left(\sigma s_{i}\right)\right]=\sum_{i=1}^{r}\left[C_{G}\left(\sigma s_{i}\right): C_{K}\left(\sigma s_{i}\right)\right] .
$$

By Lemma 2.13(i), the Shintani maps $f_{G}$ and $f_{K}$ preserve centralizer cardinalities, so

$$
\left|C_{\Omega}(\sigma s)\right|=\sum_{i=1}^{r}\left[C_{H}\left(f\left(\sigma s_{i}\right)\right): C_{L}\left(f\left(\sigma s_{i}\right)\right)\right]
$$

Finally, we observe that $f(\sigma s)^{H} \cap L$ is the union of the distinct $L$-classes $f\left(\sigma s_{i}\right)^{L}$. To see this, suppose that $l \in L$ is $H$-conjugate to $f(\sigma s)=f_{G}(\sigma s)=$ $f_{K}(\sigma s) \in L$. Since $f_{K}$ is surjective, there exists $t \in K$ such that $l=f_{K}(\sigma t)$. We may assume that $f_{G}(\sigma t)=f_{K}(\sigma t)$ and that $f_{G}(\sigma s)=f_{K}(\sigma s)$, and since $f_{G}(\sigma s)$ and $f_{G}(\sigma t)$ are $H$-conjugate, it follows that $\sigma s$ and $\sigma t$ must be $G$-conjugate elements in $\sigma K$. Thus, $\sigma t$ must be $K$-conjugate to some $\sigma s_{i}$, and therefore $l=f_{K}(\sigma t)$ must be $L$-conjugate to $f_{K}\left(\sigma s_{i}\right)$, which proves our claim. Now a further application of Lemma 2.4 yields

$$
\left|C_{\Delta}(f(\sigma s))\right|=\sum_{i=1}^{r}\left[C_{H}\left(f\left(\sigma s_{i}\right)\right): C_{L}\left(f\left(\sigma s_{i}\right)\right)\right]
$$

and thus $\left|C_{\Omega}(\sigma s)\right|=\left|C_{\Delta}(f(\sigma s))\right|$ by $(10)$.

Corollary 2.15. Let $X=A_{n-1}$, and let $Y$ be a $\sigma$-stable subgroup of $X$, where $Y$ is either a parabolic subgroup or a Levi subgroup of type $A_{i-1} A_{n-1-i} T_{1}$ with $1 \leq i<n / 2$. Assume that $f(\sigma s) \in L$ for some $s \in G$. Then the number of $H$-conjugates of $L$ containing $f(\sigma s)$ is equal to the number of $G$-conjugates of $K$ that are normalized by $\sigma s$.

Proof. First, observe that $N_{X}(Y)=Y$ and that $N_{G}(K)=K$. Now $\sigma s$ normalizes a $G$-conjugate $K^{g}$ if and only if

$$
\left(g^{-1} K g\right)^{\sigma s}=g^{-1} K g \Longleftrightarrow g^{\sigma} s g^{-1} \in N_{G}(K)=K \Longleftrightarrow K g^{\sigma} s=K g
$$


which is true if and only if $\sigma s$ fixes the coset $K g \in \Omega$. Moreover, since $N_{G}(K)=K$, we have $K^{g_{1}}=K^{g_{2}}$ if and only if $K g_{1}=K g_{2}$, and so $\left|C_{\Omega}(\sigma s)\right|$ is the number of $G$-conjugates of $K$ that are normalized by $\sigma s$. Similarly, $\left|C_{\Delta}(f(\sigma s))\right|$ is the number of $H$-conjugates of $L$ containing $f(\sigma s)$, and the result follows by Theorem 2.14 .

Corollary 2.15 will be an important tool in our later analysis. To explain how it applies, let $\sigma$ be a Frobenius morphism of $X=\operatorname{PSL}_{n}(K)$ such that $X_{\sigma^{e}}=\mathrm{PGL}_{n}(q)$ has socle $G_{0}=\operatorname{PSL}_{n}(q)$, where $K$ is the algebraic closure of $\mathbb{F}_{q}$. Now $\sigma$ induces an automorphism of $G_{0}$ (e.g., a field, or a graphfield automorphism), and we may consider the almost simple group $G=$ $\left\langle G_{0}, \sigma\right\rangle$. Fix an element $x \in X_{\sigma}$, and let $\sigma s \in \sigma X_{\sigma^{e}}$ be a representative of the corresponding $X_{\sigma^{e}}$-class under the Shintani map $f$ (see Lemma 2.13). By modifying $x$ if necessary, we may assume that $\sigma s \in \sigma G_{0}$. As before, let $\mathcal{M}(\sigma s)$ denote the set of maximal subgroups of $G$ containing $\sigma s$.

We can often use Corollary 2.15 to determine the reducible subgroups in $\mathcal{M}(\sigma s)$. For example, if $\sigma$ is a field automorphism of $G_{0}$, then the corollary tells us that there is a bijection between the set of maximal parabolic subgroups of $X_{\sigma}=\mathrm{PGL}_{n}\left(q_{0}\right)$ containing $x$ (we choose $x$ so that these subgroups are easy to identify) and the set of maximal parabolic subgroups of $G$ containing $\sigma s$. Moreover, this bijection respects the type of the parabolic subgroups involved. For example, if $x$ belongs to unique maximal parabolic subgroups of $\mathrm{PGL}_{n}\left(q_{0}\right)$ of type $P_{1}, P_{2}, P_{n-1}$, and $P_{n}$, then $\sigma s$ is contained in exactly four maximal parabolic subgroups of $G$, which again are of type $P_{1}, P_{2}, P_{n-1}$, and $P_{n}$.

The final proposition of this section provides some useful information on the nonparabolic subgroups in $\mathcal{M}(\sigma s)$.

Proposition 2.16. With the notation above, let $H \in \mathcal{M}(\sigma s)$ be a nonparabolic subgroup.

(i) There are at most $\left|C_{X_{\sigma}}(f(\sigma s))\right|$ subgroups of type $H$ in $\mathcal{M}(\sigma s)$.

(ii) Suppose that $e$ is prime, that $\sigma$ is a field automorphism, and that $H \in \mathcal{M}(\sigma s)$ is a subfield subgroup of type $\mathrm{GL}_{n}\left(q_{0}\right)$, where $q=q_{0}^{e}$. Further, assume that $f(\sigma s) \in X_{\sigma}$ is regular semisimple and either irreducible over $\mathbb{F}_{q_{0}}$ or block-diagonal of the form $[A, B]$, where $A$ and $B$ are irreducible blocks (over $\mathbb{F}_{q_{0}}$ ) of distinct dimensions. Then there are, respectively, at most e or $e^{2}$ subgroups of type $\mathrm{GL}_{n}\left(q_{0}\right)$ in $\mathcal{M}(\sigma s)$. 
Proof. Let $G_{1}=\left\langle X_{\sigma^{e}}, \sigma\right\rangle=\left\langle\operatorname{PGL}_{n}(q), \sigma\right\rangle$, and observe that all maximal subgroups of type $H$ in $G$ are $G_{1}$-conjugate (see [33, Proposition 4.0.2(i)]). In particular, if $N$ is the number of subgroups of type $H$ in $\mathcal{M}(\sigma s)$, then

$$
N=\frac{\left|(\sigma s)^{G_{1}} \cap H\right|}{\left|(\sigma s)^{G_{1}}\right|} \cdot\left[G_{1}: N_{G_{1}}(H)\right]=\frac{\left|(\sigma s)^{G_{1}} \cap H\right|\left|C_{G_{1}}(\sigma s)\right|}{\left|N_{G_{1}}(H)\right|} .
$$

To prove (i), first observe that $\left|(\sigma s)^{G_{1}} \cap H\right| \leq|H| / e$ since $(\sigma s)^{G_{1}}$ is contained in the coset $(\sigma s) \mathrm{PGL}_{n}(q)=\sigma \mathrm{PGL}_{n}(q)$, while the $(\sigma s)^{i}\left(\operatorname{PGL}_{n}(q) \cap H\right)$ (with $1 \leq i \leq e)$ are distinct cosets in $H$. Now $\left|C_{G_{1}}(\sigma s)\right|=e\left|C_{X_{\sigma}}(f(\sigma s))\right|$ by Lemma 2.13, and the result follows.

Now let $H$ be a maximal subfield subgroup of $G$ of type $\mathrm{GL}_{n}\left(q_{0}\right)$ containing $\sigma s$, where $q=q_{0}^{e}$. To prove (ii), set $H_{1}=N_{G_{1}}(H)$, and note that $\left|(\sigma s)^{G} \cap H\right| \leq\left|(\sigma s)^{G_{1}} \cap H_{1}\right|$. To estimate this upper bound, observe that some $G_{1}$-conjugate of $H_{1}$ is equal to $\langle\sigma\rangle \times X_{\sigma}$, so without loss of generality we may assume that $H_{1}=\langle\sigma\rangle \times X_{\sigma}$.

Let $\mathcal{E}=\left\{\lambda_{1}^{e}, \lambda_{2}^{e}, \ldots, \lambda_{n}^{e}\right\}$ be the set of eigenvalues of $f(\sigma s)$, and suppose that $\sigma t \in H_{1}$ is an element such that $(\sigma t)^{e}=t^{e}$ and $f(\sigma s)$ have the same eigenvalues, where $t \in X_{\sigma}$. Then $t$ has eigenvalues $\left\{\mu_{1}, \ldots, \mu_{n}\right\}$, where $\mu_{i}^{e}=$ $\lambda_{i}^{e}$ for all $1 \leq i \leq n$. In particular, there are $e$ choices for each $\mu_{i}$ (in the algebraic closure $K)$, so there are at most $e^{n}$ distinct $X_{\sigma^{-c l a s s e s}}$ in $(\sigma s)^{G_{1}} \cap$ $H_{1}$. We claim that each of these $X_{\sigma}$-classes has size $\left|f(\sigma s)^{X_{\sigma}}\right|$.

To see this, let $\sigma t \in(\sigma s)^{G_{1}} \cap H_{1}$. As above, let $\left\{\mu_{1}, \ldots, \mu_{n}\right\}$ be the set of eigenvalues of $t$, where $\mu_{i}^{e}=\lambda_{i}^{e}$ for all $i$. First, assume that $f(\sigma s)$ is irreducible over $\mathbb{F}_{q_{0}}$. Then $f(\sigma t)$ is also irreducible over $\mathbb{F}_{q_{0}}$, and so is $(\sigma t)^{e}=$ $t^{e} \in X_{\sigma}$. Further, each eigenvalue $\lambda_{i}^{e}$ of $f(\sigma s)$ is contained in $\mathbb{F}_{q_{0}^{n}}$ and in no smaller field extension of $\mathbb{F}_{q_{0}}$. Similarly, since $t \in X_{\sigma}$, the $\mu_{i}$ are also in $\mathbb{F}_{q_{0}^{n}}$ and no smaller field extension, so $t$ is irreducible over $\mathbb{F}_{q_{0}}$. Therefore,

$$
\left|C_{X_{\sigma}}(\sigma t)\right|=\left(q_{0}^{n}-1\right) /\left(q_{0}-1\right)=\left|C_{X_{\sigma}}(f(\sigma s))\right| .
$$

Moreover, since $t \in \mathrm{GL}_{n}\left(q_{0}\right)$ is irreducible over $\mathbb{F}_{q_{0}}$, the eigenvalues of $t$ must be of the form $\left\{\mu_{1}, \mu_{1}^{q_{0}}, \ldots, \mu_{1}^{q_{0}^{n-1}}\right\}$, and so in fact there are at most $e$ distinct $X_{\sigma}$-classes in $(\sigma s)^{G_{1}} \cap H_{1}$.

Now assume that $f(\sigma s)$ is block-diagonal of the form $[A, B]$, where $A$ is irreducible over $\mathbb{F}_{q_{0}}$ of dimension $n-k$ and where $B$ is irreducible of dimension $k$ (where $n-k \neq k$ ). By relabeling the eigenvalues of $f(\sigma s)$ if necessary, we may assume that $\lambda_{1}^{e}, \ldots, \lambda_{n-k}^{e}$ are contained in $\mathbb{F}_{q_{0}^{n-k}}$ (and no smaller field extension of $\mathbb{F}_{q_{0}}$ ), while the remainder are contained in $\mathbb{F}_{q_{0}^{k}}$ (and 
no smaller field extension). Now the $\lambda_{i}^{e}$ are also the eigenvalues of $(\sigma t)^{e}=t^{e}$, and it follows that either $t$ is irreducible or $t$ is also block-diagonal of the form $[C, D]$, where $C$ and $D$ are irreducible over $\mathbb{F}_{q_{0}}$ of dimensions $n-k$ and $k$, respectively. However, $t$ is not irreducible since it has eigenvalues with distinct multiplicative orders. Therefore, $t$ is of the form $[C, D]$; hence, $\left|C_{X_{\sigma}}(\sigma t)\right|=\left|C_{X_{\sigma}}(f(\sigma s))\right|$. Moreover, since the eigenvalues of $t$ are of the form

$$
\left\{\mu_{1}, \mu_{1}^{q_{0}}, \ldots, \mu_{1}^{q_{0}^{n-k-1}}, \mu_{n-k+1}, \ldots, \mu_{n-k+1}^{q_{0}^{k-1}}\right\},
$$

it follows that there are at most $e^{2}$ distinct $X_{\sigma}$-classes in $(\sigma s)^{G_{1}} \cap H_{1}$. This proves the claim.

Consequently, if $f(\sigma s)$ is irreducible, then

$$
\left|(\sigma s)^{G_{1}} \cap H_{1}\right| \leq e\left|f(\sigma s)^{X_{\sigma}}\right|=e\left[X_{\sigma}: C_{X_{\sigma}}(f(\sigma s))\right],
$$

and thus

$$
N \leq \frac{e\left|X_{\sigma}\right|\left|C_{G_{1}}(\sigma s)\right|}{\left|H_{1}\right|\left|C_{X_{\sigma}}(f(\sigma s))\right|}=\frac{e^{2}\left|X_{\sigma}\right|}{\left|H_{1}\right|}=e,
$$

since $\left|C_{G_{1}}(\sigma s)\right|=e\left|C_{X_{\sigma}}(\sigma s)\right|=e\left|C_{X_{\sigma}}(f(\sigma s))\right|$ (see Lemma 2.13). The result follows. Similarly, if $f(\sigma s)$ is of the form $[A, B]$, then we replace $e$ by $e^{2}$, and once again the result follows.

\subsection{Computational methods}

For small values of $n$ and $q$, our general techniques are less effective, and it is convenient to use a computer package such as MAGMA [4] to obtain the desired results in these situations. Here our main result is the following.

Proposition 2.17. The conclusion to Theorem 2.3 holds for all $(n, q)$ with $n \leq 10$ and $q \leq f(n)$, where $f(n)$ is defined as follows:

\begin{tabular}{|l|l|l|l|l|l|l|l|l|l|}
\hline$n$ & 2 & 3 & 4 & 5 & 6 & 7 & 8 & 9 & 10 \\
\hline$f(n)$ & 128 & 16 & 9 & 4 & 4 & 2 & 2 & 2 & 2 \\
\hline
\end{tabular}

The next result handles the exceptional cases in the statement of Theorem 3. Here $\operatorname{PSL}_{2}(9) .2 \cong S_{6}, \mathrm{PSL}_{3}(4) .2_{1}$ is an extension of $\mathrm{PSL}_{3}(4)$ by a graph-field automorphism, and $\operatorname{PSL}_{4}(3) .2_{2} \cong\left\langle\operatorname{PSL}_{4}(3), \iota\right\rangle$, where $\iota$ is the inverse-transpose graph automorphism.

Proposition 2.18. Let $G$ be one of the following groups:

$$
\mathrm{PSL}_{2}(9) .2, \quad \mathrm{PSL}_{3}(4) .2_{1}, \quad \mathrm{PSL}_{4}(2) .2, \quad \mathrm{PSL}_{4}(3) .2_{2} \text {. }
$$

Then either $u(G) \geq 2$, or $G=\mathrm{PSL}_{2}(9) .2$ and $(s(G), u(G))=(2,0)$. 
Remark 2.19. For the relevant groups $G$ in Proposition 2.18, one can check that the $G$-classes $C$ with the uniform spread 2 property are the following (here we adopt the standard $\mathbb{A} \mathbb{L} \mathbb{A} \mathbb{S}[15]$ notation for the conjugacy classes in $G)$ :

\begin{tabular}{|l|l|}
\hline$G$ & $C$ \\
\hline $\mathrm{PSL}_{3}(4) .2_{1}$ & $6 A, 8 A, 8 B, 8 C$ \\
\hline $\mathrm{PSL}_{4}(2) .2$ & $8 A$ \\
\hline $\mathrm{PSL}_{4}(3) .2_{2}$ & $6 P, 8 G, 10 B, 10 C, 12 D, 12 E, 12 F, 18 A, 18 B$ \\
\hline
\end{tabular}

To establish Propositions 2.17 and 2.18, we adopt methods similar to those used in [7], the main difference being that we use MAGMA rather than GAP. Let us briefly outline our basic approach.

Let $G=\left\langle G_{0}, g\right\rangle$, and fix an element $s \in G_{0}$. Recall that we are interested in computing

$$
\sum_{H \in \mathcal{M}(g s)} \operatorname{fpr}(x, G / H)
$$

where $x \in G$ has prime order and $\mathcal{M}(g s)$ is the set of maximal subgroups in $G$ containing $g s$. First, we use MAgma to construct $G_{0}$ as a permutation group on $\left(q^{n}-1\right) /(q-1)$ points. (This is the representation of $G_{0}$ on the set of cosets of a maximal parabolic subgroup $P_{1}$; it is the standard representation of $\operatorname{PSL}_{n}(q)$ in MAGMA.) We then use the MAgMA commands AutomorphismGroup (which is based on the algorithm of Cannon and Holt [13]) and PermutationGroup to obtain $\operatorname{Aut}\left(G_{0}\right)$ as a permutation group of reasonable degree. This is effective in most of the cases we consider in Proposition 2.17. However, if $G_{0}$ is large, say, $G_{0}=\operatorname{PSL}_{10}(2)$ or $\operatorname{PSL}_{6}(4)$, then it is much more efficient to construct $\operatorname{Aut}\left(G_{0}\right)$ directly, using the natural permutation representation of $\mathrm{PCL}_{n}(q)=\left\langle\operatorname{PGL}_{n}(q), \phi\right\rangle$ on $\left(q^{n}-1\right) /(q-1)$ points. (This is the subgroup of $\operatorname{Aut}\left(G_{0}\right)$ generated by the inner, diagonal, and field automorphisms of $G_{0}$.) To do this, we first construct the direct product $A=\mathrm{P} L_{n}(q) \times \mathrm{P} L_{n}(q)$ and the subgroup $B=\left\{\left(\phi x, \phi x^{-T}\right) \mid x \in \mathrm{PGL}_{n}(q)\right\}$ of $A$. Note that $B \cong \mathrm{P \Gamma L}_{n}(q)$ and that the inverse-transpose automorphism $\iota$ acts on $B$ by swapping $\phi x$ and $\phi x^{-T}$. This gives $\operatorname{Aut}\left(G_{0}\right) \cong\langle B, \iota\rangle$ as a permutation group of degree $2\left(q^{n}-1\right) /(q-1)$.

Next, we identify $G$ as a suitable subgroup of $\operatorname{Aut}\left(G_{0}\right)$ (with the aid of the command LowlndexSubgroups), and we compute representatives of the conjugacy classes of both elements and maximal subgroups of $G$ via 
the commands ConjugacyClasses and MaximalSubgroups, respectively. (The latter denotes the MAGMA implementation of an algorithm of Cannon and Holt [14].)

Let $\mathcal{C}$ be a set of representatives of the $G$-classes of maximal subgroups of $G$, and let $H \in \mathcal{C}$. It is straightforward to calculate the fixed point ratio

$$
\operatorname{fpr}(x, G / H)=\frac{\left|x^{G} \cap H\right|}{\left|x^{G}\right|}
$$

for all $x \in G$. Indeed, we first compute a set of representatives for the $H$ classes of $H$, and then we add up the lengths of the classes that are represented by $G$-conjugates of $x$. (These are determined using the IsConjugate command.) This gives $\left|x^{G} \cap H\right|$, and $\operatorname{fpr}(x, G / H)$ quickly follows.

For each $H \in \mathcal{C}$, let $N_{H}$ be the number of distinct $G$-conjugates of $H$ containing $g s$. Then

$$
N_{H}=\operatorname{fpr}(g s, G / H) \cdot[G: H],
$$

and we can compute

$$
\alpha(x):=\sum_{H \in \mathcal{M}(g s)} \operatorname{fpr}(x, G / H)=\sum_{H \in \mathcal{C}} N_{H} \cdot \operatorname{fpr}(x, G / H)
$$

for all $x \in G$ of prime order. If we can find an element $g s \in g G_{0}$ such that the maximum value of $\alpha(x)$ is less than $1 / 2$ (as we run through a set of $G$-class representatives of prime order), then $u(G) \geq 2$.

For the vast majority of the groups that we consider, in Proposition 2.17 there exists a suitable element $g s$ with $\alpha(x)<1 / 2$ for all $x \in G$ of prime order. Indeed, the only exceptions are the groups appearing in the statement of Proposition 2.18. For these groups, we attempt to show that $u(G) \geq 2$ directly (which is expensive). Our strategy is to choose a good candidate $g s \in G$ (based on the elements we use in the proof of Theorem 2.3 in Sections 3-6), and then we check that for all nontrivial $x_{1}, x_{2} \in G$, there exists $y \in$ $(g s)^{G}$ such that

$$
G=\left\langle x_{1}, y\right\rangle=\left\langle x_{2}, y\right\rangle
$$

Of course, here we may assume that $x_{1}$ and $x_{2}$ have prime order. Further, it is easy to see that it suffices to check that (11) holds when $x_{2}$ belongs to a set of representatives of the $G$-classes containing elements of prime order. 


\section{§3. Diagonal automorphisms}

Let $G_{0}=\operatorname{PSL}_{n}(q)$, where $n \geq 2$ and $q=p^{f}$ for a prime $p$. Recall from Section 2.1 that every automorphism of $G_{0}$ is a product of the form $i d f g$, where $i$ is inner, $d$ is diagonal, and $f$ and $g$ are field and graph automorphisms, respectively. More precisely,

$$
\operatorname{Aut}\left(G_{0}\right)=\left(G_{0} \rtimes Z_{(n, q-1)}\right) \rtimes\left(Z_{f} \times Z_{a}\right),
$$

where $a=2$ if $n \geq 3$; otherwise, $a=1$. Consequently, in order to prove Theorems 2-4, we may assume that $G=\left\langle G_{0}, g\right\rangle$, where $g \in \operatorname{Aut}\left(G_{0}\right)$ is one of the following:

(i) $g \in \mathrm{PGL}_{n}(q)$ is a diagonal automorphism;

(ii) $g=\sigma x$, where $\sigma$ is a nontrivial field automorphism and $x \in \operatorname{PGL}_{n}(q)$;

(iii) $g=\iota \sigma x$, where $\iota$ is the inverse-transpose graph automorphism, $\sigma$ is a nontrivial field automorphism, and $x \in \operatorname{PGL}_{n}(q)$;

(iv) $g=\iota x$, where $\iota$ is the inverse-transpose graph automorphism and $x \in$ $\operatorname{PGL}_{n}(q)$.

TheOREM 3.1. Theorems 2-4 hold in case (i).

Proof. The proof of [7, Theorem 1.1] provides an explicit semisimple element $s \in G_{0}$ such that

$$
\mathbb{P}\left(G_{0}=\langle x, y\rangle \mid y \in s^{G_{0}}\right)>2 / 3
$$

for all nontrivial $x \in G_{0}$. Moreover, since $G \leq \mathrm{PGL}_{n}(q)$, we observe that there exists $s_{1} \in G$ such that $G=\left\langle G_{0}, s_{1}\right\rangle$ and $s_{1}^{m}=s$ for some integer $m$. The proof of the above bound in [7] now goes through unchanged (see, e.g., [7, Proposition 5.23]), and we conclude that

$$
\mathbb{P}\left(G=\langle x, y\rangle \mid y \in s_{1}^{G}\right)>2 / 3
$$

for all nontrivial $x \in G$. Therefore, Theorem 3 holds and Theorem 2 follows in the usual way. (Note that $u(G) \geq 3$ in this case.)

Now let us turn to Theorem 4. In [23, Sections 4, 5] it is proved that if $G_{i}$ is a sequence of simple groups isomorphic to $\operatorname{PSL}_{n_{i}}\left(q_{i}\right)$, then $u\left(G_{i}\right) \rightarrow \infty$ if $\left|G_{i}\right| \rightarrow \infty$ (see also [27, Propositions 3.6 and 3.9]). As in [7], an explicit semisimple element $s \in G_{0}=\operatorname{PSL}_{n}(q)$ is given in [23], and it is shown that $\mathbb{P}\left(G_{0}=\langle x, y\rangle \mid y \in s^{G_{0}}\right)$ is bounded below by a function of $n$ and $q$, which tends to 1 as $n$ or $q$ tend to infinity. In particular, if $\operatorname{PSL}_{n_{i}}\left(q_{i}\right)<G_{i} \leq$ $\mathrm{PGL}_{n_{i}}\left(q_{i}\right)$, then we can choose $s_{1} \in G_{i}$ as in the previous paragraph so that the argument in [23] also yields $u\left(G_{i}\right) \rightarrow \infty$. 
We will deal with cases (ii)-(iv) in the next three sections.

\section{§4. Field automorphisms}

In this section we consider the case $G=\left\langle G_{0}, g\right\rangle$, where $g=\sigma x$, with $\sigma$ a field automorphism of $G_{0}$ of order $e>1$ and $x \in \mathrm{PGL}_{n}(q)$. Here $q=q_{0}^{e}$ for some $p$-power $q_{0}$, and by fixing a suitable basis for the natural $G_{0}$-module $V$, we may assume that $\sigma$ is standard in the sense that $\sigma:\left(a_{i j}\right) \mapsto\left(a_{i j}^{q_{0}}\right)$. In addition, we may write $x=\delta t$, where $t \in G_{0}$ and $\delta$ is a diagonal matrix of the form $\delta=\left[\lambda, I_{n-1}\right]$ (modulo scalars) for some $\lambda \in \mathbb{F}_{q}^{*}$. Therefore, $G=$ $\left\langle G_{0}, g\right\rangle=\left\langle G_{0}, \sigma \delta\right\rangle$, so without any loss of generality we may assume that $g=\sigma \delta$.

The main result of this section is the following. (Recall that $\mathcal{M}(g s)$ is the set of maximal subgroups of $G$ containing $g s$.

Theorem 4.1. Let $G_{0}=\operatorname{PSL}_{n}(q)$, and let $G=\left\langle G_{0}, g\right\rangle$, where $g=\sigma x$, with $\sigma$ a nontrivial field automorphism of $G_{0}$ and $x \in \operatorname{PGL}_{n}(q)$. Assume that $G \neq \mathrm{PSL}_{2}(9) .2$. Then there exists $s \in G_{0}$ such that

$$
\sum_{H \in \mathcal{M}(g s)} \operatorname{fpr}(z, G / H)<1 / 2
$$

for all $z \in G$ of prime order. In particular, $u(G) \geq 2$, and $u(G) \rightarrow \infty$ as $|G| \rightarrow \infty$.

Our approach is based on the theory of Shintani descent (see Section 2.6). Let $X=\mathrm{PSL}_{n}(K)$ be the ambient simple algebraic group over the algebraic closure $K$ of $\mathbb{F}_{q}$. We may view $\sigma$ as a Frobenius morphism of $X$ with fixed point subgroups $X_{\sigma}=\mathrm{PGL}_{n}\left(q_{0}\right)$ and $X_{\sigma^{e}}=\mathrm{PGL}_{n}(q)$. By Lemma 2.13, the corresponding Shintani map $f$ provides a bijection between the set of $\mathrm{PGL}_{n}(q)$-classes in the coset $\sigma \mathrm{PGL}_{n}(q)$ and the set of $\mathrm{PGL}_{n}\left(q_{0}\right)$-classes in $\mathrm{PGL}_{n}\left(q_{0}\right)$. As before, for $s \in G_{0}$ we abuse notation by writing $f(\sigma s)$ for a representative of the $\mathrm{PGL}_{n}\left(q_{0}\right)$-class corresponding to the $\mathrm{PGL}_{n}(q)$-class of $\sigma s$, so $f(\sigma s)$ is $X$-conjugate to $(\sigma s)^{e}$. In view of (6), we note that if $s \in G_{0}$, then $f(g s)=f(\sigma \delta s)$ has determinant $\lambda^{\alpha}$, where $\alpha=(q-1) /\left(q_{0}-1\right)$.

Lemma 4.2. Suppose that $y \in \mathrm{PGL}_{n}\left(q_{0}\right)$ has determinant $\lambda^{\alpha}$. Then there exists $s \in G_{0}$ such that $f(g s)$ is $\mathrm{PGL}_{n}\left(q_{0}\right)$-conjugate to $y$.

Proof. Since the Shintani map $f$ is a bijection, the $\mathrm{PGL}_{n}\left(q_{0}\right)$-class of $y$ corresponds to the $\mathrm{PGL}_{n}(q)$-class of $\sigma t$ for some $t \in \mathrm{PGL}_{n}(q)$. Let $\mu$ be 
the determinant of $t$, and fix a generator $\omega$ for $\mathbb{F}_{q}^{*}$. Since $f(\sigma t)$ and $y$ are $\mathrm{PGL}_{n}\left(q_{0}\right)$-conjugate, it follows that $\mu^{\alpha}=\lambda^{\alpha}$, and thus $\mu=\omega^{\left(q_{0}-1\right) j} \lambda$ for some integer $0 \leq j<\alpha$. Let $x \in \mathrm{PGL}_{n}(q)$ be an element with determinant $\omega^{j}$. Then $(\sigma t)^{x}=\sigma x^{-\sigma} t x$ and $x^{-\sigma} t x$ has determinant $\lambda$. Therefore, $x^{-\sigma} t x \in$ $\delta G_{0}$, so there exists $s \in G_{0}$ such that $\sigma x^{-\sigma} t x=\sigma \delta s=g s \in G$ corresponds to $y$ under the Shintani correspondence.

We also need the following number-theoretical result. In the statement, for a positive integer $n$ we write $n_{2}$ for the largest power of 2 dividing $n$. In addition, recall that $(a, b)$ denotes the greatest common divisor of the positive integers $a$ and $b$.

Lemma 4.3. Let $q \geq 2$ be an integer. For all integers $n, m \geq 1$, we have

$$
\begin{aligned}
& \left(q^{n}-1, q^{m}-1\right)=q^{(n, m)}-1 ; \\
& \left(q^{n}-1, q^{m}+1\right)= \begin{cases}q^{(n, m)}+1 & \text { if } 2 m_{2} \leq n_{2}, \\
(2, q-1) & \text { otherwise; }\end{cases} \\
& \left(q^{n}+1, q^{m}+1\right)= \begin{cases}q^{(n, m)}+1 & \text { if } m_{2}=n_{2}, \\
(2, q-1) & \text { otherwise. }\end{cases}
\end{aligned}
$$

Proof. This is a straightforward calculation.

Proposition 4.4. Theorem 4.1 holds when $n \geq 5$.

Proof. Set $y=\left[J_{2}, A\right] \in \mathrm{PGL}_{n}\left(q_{0}\right)$, where $J_{2}$ denotes a standard unipotent Jordan block of size 2 and $A \in \mathrm{GL}_{n-2}\left(q_{0}\right)$ is a semisimple irreducible element with determinant $\lambda^{\alpha}$, where $\alpha=(q-1) /\left(q_{0}-1\right)$ as before. More precisely, we take $A$ to be a suitable power of a Singer cycle in $\mathrm{GL}_{n-2}\left(q_{0}\right)$ of order $q_{0}^{n-2}-1$, so $A$ has order $\left(q_{0}^{n-2}-1\right)\left|\lambda^{\alpha}\right| /\left(q_{0}-1\right)$, where $\left|\lambda^{\alpha}\right|$ denotes the multiplicative order of $\lambda^{\alpha}$ in the cyclic group $\mathbb{F}_{q_{0}}^{*}$. Note that if $\left(n, q_{0}\right) \neq(8,2)$, then the order of some suitable power of $y$ is a primitive prime divisor of $q_{0}^{n-2}-1$ (see Section 2.5). In addition, $y$ has determinant $\lambda^{\alpha}$, so by Lemma 4.2 there exists $s \in G_{0}$ such that the corresponding Shintani map sends the $\mathrm{PGL}_{n}(q)$-class of $g s$ to the $\mathrm{PGL}_{n}\left(q_{0}\right)$-class of $y$. In particular, $y$ and $(g s)^{e}$ are $X$-conjugate. We can write

$$
\mathcal{E}=\left\{1, \omega^{k}, \omega^{q_{0} k}, \omega^{q_{0}^{2} k}, \ldots, \omega^{q_{0}^{n-3} k}\right\}
$$

for the set of eigenvalues of $(g s)^{e}$, where $\omega$ is a generator of $\mathbb{F}_{q_{0}^{n-2}}^{n}$ and $k=\left(q_{0}-1\right) /\left|\lambda^{\alpha}\right|$. 
Our first task is to determine the maximal subgroups of $G$ containing $g s$; as before, we write $\mathcal{M}=\mathcal{M}(g s)$ to denote this set of subgroups. (In part (i) of the following lemma we use $P_{i}$ to denote the $G$-stabilizer of an $i$-dimensional subspace of the natural $G_{0}$-module.)

Lemma 4.5. Suppose that $H \in \mathcal{M}$. Then one of the following holds.

(i) $\quad H$ is a maximal parabolic subgroup of type $P_{1}, P_{2}, P_{n-2}$, or $P_{n-1}$, and there is exactly one subgroup of each type in $\mathcal{M}$.

(ii) $H$ is an imprimitive $\mathcal{C}_{2}$-subgroup, and one of the following holds.

(a) $H$ is of type $\mathrm{GL}_{2}(q) 2 S_{n / 2}$, and $e \geq(n-2) / 2$. There is a unique subgroup of this type in $\mathcal{M}$.

(b) $H$ is of type $\mathrm{GL}_{1}(q) 2 S_{n}$, where $q$ is even, e is odd, and $e \geq n-2$. There are at most $q_{0} / 2$ subgroups of this type in $\mathcal{M}$.

(iii) $H$ is a subfield subgroup of type $\mathrm{GL}_{n}\left(q_{1}\right)$, where $q=q_{1}^{r}$, with $r$ a prime divisor of e. For each prime $r$, there are at most $q_{0}\left(q_{0}^{n-2}-1\right)$ corresponding subfield subgroups in $\mathcal{M}$.

Proof. By Corollary 2.15, there is a bijective correspondence between the reducible subgroups in $\mathcal{M}$ and the reducible subgroups of $\operatorname{PGL}_{n}\left(q_{0}\right)$ containing $y$. Therefore, the maximal parabolic subgroups $P_{1}, P_{2}, P_{n-2}$, and $P_{n-1}$ are the only possibilities. Moreover, there is exactly one subgroup in $\mathcal{M}$ of each type since $y$ clearly fixes a unique $i$-dimensional subspace of the natural $\mathrm{PGL}_{n}\left(q_{0}\right)$-module for each $i \in\{1,2, n-2, n-1\}$.

For the remainder, let us assume that $H \in \mathcal{M}$ is irreducible. Recall from Section 2.3 that a maximal irreducible subgroup of $G$ belongs to one of eight subgroup collections, labeled $\mathcal{C}_{i}$ (where $2 \leq i \leq 9$ ). From the Shintani setup, $(g s)^{e}$ is $X$-conjugate to $y$, so a suitable power of $(g s)^{e}$, say, $z=(g s)^{m^{\prime} e}$, is a long root element (i.e., a transvection $\left[J_{2}, I_{n-2}\right]$ ). This useful observation allows us to restrict significantly the possibilities for $H$.

Suppose that $H$ is a field extension subgroup of type $\operatorname{GL}_{n / k}\left(q^{k}\right)$ for some prime $k$. (Recall that these subgroups comprise Aschbacher's $\mathcal{C}_{3}$ collection.) By the proof of $[38$, Lemma 4.2], we have $\nu(x) \geq k$ for all $x \in H \cap \operatorname{PGL}(V)$ of prime order (see $(4))$, so $z \notin H$ since $\nu(z)=1$. Similarly, by applying [38, Lemma 3.7], we deduce that there are no $\mathcal{C}_{4^{-}}$or $\mathcal{C}_{7}$-subgroups in $\mathcal{M}$, while $\mathcal{C}_{6}$-subgroups are ruled out by $[10$, Lemma 6.3$]$.

Next, let us turn our attention to the imprimitive $\mathcal{C}_{2}$-subgroups in $\mathcal{M}$. If $\left(n, q_{0}\right) \neq(8,2)$, then a suitable power of $y$, say, $y^{m}$, has order $r$, where $r$ is a primitive prime divisor of $q_{0}^{n-2}-1$. Set $x=(g s)^{m e}$, so $x$ has order 
$r$, and note that $r \geq n-1$ since $r \equiv 1(\bmod n-2)$. On the other hand, if $\left(n, q_{0}\right)=(8,2)$, then $y^{18}$ has order 7 , and we set $x=(g s)^{18 e}$.

Suppose that $H \in \mathcal{M}$ is of type $\mathrm{GL}_{n / t}(q)$ ? $S_{t}$ with $t \geq 2$ an integer dividing $n$, so $H$ is the $G$-stabilizer of a decomposition of $V$ of the form

$$
V=V_{1} \oplus V_{2} \oplus \cdots \oplus V_{t}
$$

where $\operatorname{dim} V_{i}=n / t$ for all $i$.

First, we claim that $x$ fixes each of the subspaces in the above decomposition (13). To see this, suppose that $x$ induces a nontrivial permutation $\pi \in S_{t}$ on the $V_{i}$. Since $x$ has prime order $r \geq n-1$, it follows that $n=t$ and that $r \in\{n-1, n\}$. Further, since $z$ is a transvection, it induces a nontrivial permutation $\rho \in S_{t}$ on the 1 -spaces. (In fact, we must have $p=2$ with $\rho$ a transposition.) Now $\rho$ and $\pi$ commute (since $x$ and $z$ are both powers of $g s$ ), but this is a contradiction since $C_{S_{t}}(\pi)=\langle\pi\rangle$. This justifies the claim.

Next, we reduce to the case $\operatorname{dim} V_{i} \leq 2$. To do this, first observe that $x$ and $(g s)^{e}$ commute, so $(g s)^{e}$ fixes each of the eigenspaces of $x$ (over the algebraic closure $K)$. Further, $x$ is semisimple with $n-1$ distinct eigenvalues (1 occurs with multiplicity 2$)$, so $(g s)^{e}$ fixes all of the subspaces in the decomposition (13) on which $x$ acts nontrivially. (Also recall that $x$ fixes each $V_{i}$ by the previous claim.) Seeking a contradiction, let us assume that $\operatorname{dim} V_{i} \geq 3$. Here $x$ acts nontrivially on each $V_{i}$, so $(g s)^{e}$ fixes each $V_{i}$. Next, observe that if $\xi \in \mathbb{F}_{q}$ is an eigenvalue of $(g s)^{e}$, then $g s=\sigma \delta s$ sends a corresponding $\xi$-eigenvector to a $\xi^{q_{0}}$-eigenvector of $(g s)^{e}$. Indeed, if $v \in V_{i}$ is a $\xi$-eigenvector for $(g s)^{e}$, then

$$
(v \cdot g s) \cdot(g s)^{e}=\left(v \cdot(g s)^{e}\right) \cdot g s=(\xi v) \cdot g s=\xi^{q_{0}}(v \cdot g s) .
$$

In particular, gs maps 1-eigenvectors to 1-eigenvectors. Without loss of generality, we may assume that $V_{1}$ is a subspace containing a 1-eigenvector. Since the geometric multiplicity of 1 as an eigenvalue of $(g s)^{e}$ on $V$ is 1 , it follows that the algebraic multiplicity of 1 as an eigenvalue of $(g s)^{e}$ on $V_{1}$ is 2 . Therefore, $g s$ fixes $V_{1}$, but this contradicts our earlier observation that the only parabolic subgroups containing $g s$ are of type $P_{1}, P_{2}, P_{n-1}$, and $P_{n}$ (obtained via Corollary 2.15). For the remainder, we may assume that $\operatorname{dim} V_{i} \leq 2$.

First, assume that $\operatorname{dim} V_{i}=1$. Here $H$ is of type $\mathrm{GL}_{1}(q) 2 S_{n}$, and we note that $q$ is even since $H$ contains the transvection $z=(g s)^{m^{\prime} e}$. Recall that 
$(g s)^{e}$ fixes each $V_{i}$ in (13) on which $x$ acts nontrivially, so all the eigenvalues of $(g s)^{e}$ are in $\mathbb{F}_{q}$. (Hence, $e \geq n-2$ since the eigenvalues of $(g s)^{e}$ are contained in $\mathbb{F}_{q_{0} n-2}$ and in no proper subfield (see (12)).) In particular, the $V_{i}$ are simply the eigenspaces of $(g s)^{e}$ corresponding to the $n-2$ eigenvalues $\xi \in \mathbb{F}_{q}$ with $\xi \neq 1$, together with the 2-dimensional fixed space of $x$, say, $C_{V}(x)=V_{1} \oplus V_{2}$. Now $z$ (and therefore $(g s)^{e}$ and also $g s$ ) interchanges $V_{1}$ and $V_{2}$, and so $e$ must be odd.

We claim that there are precisely $q_{0} / 2$ distinct possibilities for the 1 spaces $\left\{V_{1}, V_{2}\right\}$ in the 2-dimensional fixed space of $x$, so $g s$ can belong to at most $q_{0} / 2$ distinct $\mathcal{C}_{2}$-subgroups of type $\mathrm{GL}_{1}(q)$ ₹ $S_{n}$. Fix an $\mathbb{F}_{q}$-basis $\{u, v\}$ for $C_{V}(x)$, and suppose that $V_{1}=\langle a u+b v\rangle$ and that $V_{2}=\langle c u+d v\rangle$ for some $a, b, c, d \in \mathbb{F}_{q}$. Since $(g s)^{2}$ fixes $V_{1}$ and $V_{2}$, we may assume that $a, b, c, d \in \mathbb{F}_{q_{0}}$. (Note that $e$ is odd and that $\langle\xi(a u+b v)\rangle=\langle a u+b v\rangle,\langle\xi(c u+$ $d v)\rangle=\langle c u+d v\rangle$ for all $\xi \in \mathbb{F}_{q}^{*}$.) Evidently, there are $q_{0}+1$ possibilities for $V_{1}$ : either $V_{1}=\langle u+\xi v\rangle$ for some $\xi \in \mathbb{F}_{q_{0}}$, or $V_{1}=\langle v\rangle$. Now $q_{0}$ is even and $(g s)^{e}$ interchanges $V_{1}$ and $V_{2}$, so there are $q_{0} / 2$ possibilities for the pair $\left\{V_{1}, V_{2}\right\}$, as claimed.

To complete the analysis of $\mathcal{C}_{2}$ subgroups, let us assume that $\operatorname{dim} V_{i}=2$. By Corollary 2.15, gs belongs to a unique maximal parabolic subgroup of $G$ of type $P_{2}$, so there is a unique 2-dimensional subspace of $V$ fixed by $g s$. Recall that $(g s)^{e}$ fixes each $V_{i}$ on which $x$ acts nontrivially. Clearly, either $x$ acts nontrivially on each $V_{i}$, or one of the $V_{i}$ coincides with $C_{V}(x)$. It follows that each eigenvalue of $(g s)^{e}$ belongs to $\mathbb{F}_{q^{2}}$ (and thus $e \geq(n-2) / 2$ ). Now, if every nontrivial eigenvalue of $x$ belongs to $\mathbb{F}_{q^{2}} \backslash \mathbb{F}_{q}$, then the $V_{i}$ are 2-spaces fixed by $(g s)^{e}$. In particular, the decomposition is unique, and thus $g s$ belongs to a unique $\mathcal{C}_{2}$-subgroup of type $\mathrm{GL}_{2}(q)$ i $S_{n / 2}$. Finally, let us assume that all the eigenvalues of $(g s)^{e}$ are in $\mathbb{F}_{q}$. By Galois theory, $g s$ acts transitively on the set of roots of the minimal polynomial of $(g s)^{e}$ that are not equal to 1 , which immediately implies that $g s$ acts transitively on the $n-2$ nontrivial eigenvalues of $(g s)^{e}$. In particular, $g s$ induces an $(n-2)$-cycle on the corresponding eigenspaces $\left\{\left\langle v_{i}\right\rangle \mid 1 \leq i \leq n-2\right\}$, so there is a unique $g s$-invariant partition of $\left\langle v_{1}\right\rangle \oplus \cdots \oplus\left\langle v_{n-2}\right\rangle$ into 2-spaces. The remaining 2-space is $C_{V}(x)$, so $g s$ fixes a unique decomposition of type (13), and once again we conclude that $g s$ belongs to a unique $\mathcal{C}_{2}$-subgroup of type $\mathrm{GL}_{2}(q)$ ? $S_{n / 2}$.

We have now dealt with the subgroups in the $\mathcal{C}_{1}, \mathcal{C}_{2}, \mathcal{C}_{3}, \mathcal{C}_{4}, \mathcal{C}_{6}$, and $\mathcal{C}_{7}$ collections. Clearly, there are no $\mathcal{C}_{8}$-subgroups of type $O_{n}^{\epsilon}(q)$ (with $q$ odd) in $\mathcal{M}$ since these subgroups do not contain transvections. To eliminate any 
subgroups in the $\mathcal{C}_{9}$ collection, we can either appeal to [26, Theorem 7.1$]$ (if $n \geq 6$ ) or use the main theorem of [30]. In this way, we deduce that any additional $H \in \mathcal{M}$ is one of the following:

(i) $\quad H$ is a $\mathcal{C}_{5}$-subgroup of type $\mathrm{GL}_{n}\left(q_{1}\right)$, with $q=q_{1}^{r}$ for some prime $r$

(ii) $H$ is a $\mathcal{C}_{8}$-subgroup of type $\operatorname{Sp}_{n}(q)$, with $n$ even;

(iii) $H$ is a $\mathcal{C}_{8}$-subgroup of type $\mathrm{GU}_{n}\left(q^{1 / 2}\right)$.

We claim that there are no subgroups in $\mathcal{M}$ of type (ii) or (iii). To see this, first suppose that $H \in \mathcal{M}$ is a $\mathcal{C}_{8}$-subgroup of type $\operatorname{Sp}_{n}(q)$. Recall that the set of eigenvalues of $(g s)^{e}$ is given in (12), where $\mathbb{F}_{q_{0}^{n-2}}^{*}=\langle\omega\rangle$ and $k=\left(q_{0}-1\right) /\left|\lambda^{\alpha}\right|$. The presence of the $J_{2}$ block in $y$ implies that $(g s)^{e}$ is in $\operatorname{PSp}_{n}(q)$, so we must have $\xi^{-1} \in \mathcal{E}$ for all $\xi \in \mathcal{E}$. However, $\omega^{-k} \notin \mathcal{E}$. Indeed, if there exists $1 \leq i \leq n-3$ such that $\omega^{-k}=\omega^{q_{0}^{i} k}$, then $q_{0}^{i} k \equiv-k$ $\left(\bmod q_{0}^{n-2}-1\right)$, and thus $q_{0}^{n-2}-1$ divides $\left(q_{0}^{i}+1\right) k$. This is a contradiction since $k \leq q_{0}-1$. We conclude that there are no subgroups of type $\operatorname{Sp}_{n}(q)$ in $\mathcal{M}$.

Now suppose that $H \in \mathcal{M}$ is a $\mathcal{C}_{8}$-subgroup of type $\mathrm{GU}_{n}\left(q^{1 / 2}\right)$. Then $q$ is an even power of $p$, and $\xi^{-q^{1 / 2}} \in \mathcal{E}$ for all $\xi \in \mathcal{E}$. In particular, there exists a nonnegative integer $i \leq n-3$ such that $\omega^{-q_{0}^{f} k}=\omega^{q_{0}^{i} k}$, where $f=e / 2$, so $q_{0}^{n-2}-1$ divides $k\left(q_{0}^{f}+q_{0}^{i}\right)$. Since $k \leq q_{0}-1$, we have $q_{0}^{f} \geq q_{0}^{n-3}$, and thus $f \geq n-3 \geq i$. But $\left(q_{0}^{n-2}-1, k\left(q_{0}^{f}+q_{0}^{i}\right)\right) \leq k\left(q_{0}^{n-2}-1, q_{0}^{f-i}+1\right)$, and by Lemma 4.3 this is at most $k\left(2, q_{0}-1\right)$ unless $2(f-i)_{2} \leq(n-2)_{2}$, in which case it is at most $k\left(q_{0}^{(f-i, n-2)}+1\right) \leq k\left(q_{0}^{(n-2) / 2}+1\right)<q_{0}^{n-2}-1$. This contradicts the fact that $q_{0}^{n-2}-1$ divides $k\left(q_{0}^{f}+q_{0}^{i}\right)$, so there are no subgroups of type $\mathrm{GU}_{n}\left(q^{1 / 2}\right)$ in $\mathcal{M}$.

Finally, suppose that $H \in \mathcal{M}$ is a subfield subgroup of type $\operatorname{GL}_{n}\left(q_{1}\right)$, where $q=q_{1}^{r}$ with $r$ prime. Since $y \in \operatorname{PGL}_{n}\left(q_{1}\right) \cap \operatorname{PGL}_{n}\left(q_{0}\right)$, we quickly deduce that $\mathbb{F}_{q_{0}} \cap \mathbb{F}_{q_{1}}=\mathbb{F}_{q_{0}}$, so $q_{1}=q_{0}^{m}$ for some $m \geq 1$. Therefore, $e=m r$ (recall that $q=q_{0}^{e}$ ), and thus the number of possibilities for $r$ is equal to the number of distinct prime divisors of $e$ (which is less than $\log (e)+1$ ). By Proposition 2.16(i), there are at most $\left|C_{\mathrm{PGL}_{n}\left(q_{0}\right)}(y)\right|=q_{0}\left(q_{0}^{n-2}-1\right)$ subfield subgroups of type $\mathrm{GL}_{n}\left(q_{1}\right)$ in $\mathcal{M}$.

We are now in a position to proceed with the proof of Proposition 4.4. Let $z \in G$ be an element of prime order, and set

$$
\alpha(z)=\sum_{H \in \mathcal{M}} \operatorname{fpr}(z, G / H) .
$$


For the parabolic subgroups in $\mathcal{M}$, Theorem 2.7 gives $\operatorname{fpr}(z, G / H)<q^{-1}+$ $q^{1-n}$ if $H$ is of type $P_{1}$ or $P_{n-1}$, and $\operatorname{fpr}(z, G / H)<2 q^{-2}$ if $H$ is of type $P_{2}$ or $P_{n-2}$. Therefore, the contribution to $\alpha(z)$ from the reducible subgroups in $\mathcal{M}$ is less than

$$
2 q^{-1}+2 q^{1-n}+4 q^{-2}
$$

For the remaining subgroups $H \in \mathcal{M}$, Corollary 2.9 states that

$$
\operatorname{fpr}(z, G / H)<\left(\frac{\left(q^{n-1}-1\right)\left(q^{n}-1\right)}{q-1}\right)^{-(1 / 2)+(1 / n)}=f(n, q) .
$$

In view of Lemma 4.5, using the fact that there are less than $\log (e)+1$ distinct prime divisors of $e$, we conclude that

$$
\begin{aligned}
\alpha(z)< & 2 q^{-1}+2 q^{1-n}+4 q^{-2} \\
& +\left(\frac{1}{2} q_{0}+1+(\log (e)+1) q_{0}\left(q_{0}^{n-2}-1\right)\right) \cdot f(n, q) .
\end{aligned}
$$

If $e \geq 3$, then this bound implies that $\alpha(z)<1 / 2$ unless $\left(n, q_{0}, e\right)=$ $(5,2,3)$. Here we may omit the term $\log (e)$, and this gives $\alpha(z)<1 / 2$ as required. Similarly, if $e=2$ and $q_{0} \geq 3$, then the above bound (again, with $\log (e)$ omitted) is sufficient unless $\left(n, q_{0}\right)=(5,3)$.

Suppose that $\left(n, q_{0}, e\right)=(5,3,2)$. As above, we calculate that the contribution to $\alpha(z)$ from nonsubfield subgroups is less than 0.285 . Therefore, we need to show that the remaining contribution, which we will denote by $\beta(z)$, is at most 0.215 . Let $H \in \mathcal{M}(g s)$ be a subfield subgroup of type $\mathrm{GL}_{5}(3)$. Since $\beta(z) \leq 3\left(3^{3}-1\right) \cdot \operatorname{fpr}(z, G / H)$, it suffices to show that $\operatorname{fpr}(z, G / H) \leq$ 0.0027 for all $z \in H$ of prime order. This is a straightforward calculation. For example, suppose that $z \in H$ is semisimple. If $\nu(z) \geq 2$ (see (4)), then $\left|z^{G}\right|>(1 / 2) 3^{12}$ by [10, Corollary 3.38], and the desired bound follows from Theorem 2.6. Also, if $\nu(z)=1$, then

$$
\operatorname{fpr}(z, G / H)=\frac{\left|z^{H}\right|}{\left|z^{G}\right|}=\frac{\left|\mathrm{GL}_{5}(3)\right|}{\left|\mathrm{GL}_{4}(3)\right|\left|\mathrm{GL}_{1}(3)\right|} \cdot \frac{\left|\mathrm{GL}_{4}(9)\right|\left|\mathrm{GL}_{1}(9)\right|}{\left|\mathrm{GL}_{5}(9)\right|}=\frac{1}{4941}
$$

The other cases are very similar.

Next, suppose that $q=4$. If $n=5$ or 6 , then Proposition 2.17 applies, so we will assume that $n \geq 7$. Let $k<n / 2$ be maximal such that $n-k$ is odd and $(n, n-k)=1$. We claim that $k \geq n / 4$. If $n$ is even, then take $k$ to be a prime in the range $n / 4<k<n / 2$ (such a prime exists by Bertrand's 
postulate), so $n-k$ is odd and $(n, n-k)=1$. Now suppose that $n$ is odd. (We may as well assume that $n$ is reasonably large, say, $n>100$.) Let $k^{\prime}$ be a prime in the range $n / 8<k^{\prime}<n / 4$, and set $k=2 k^{\prime}$. Then $n-k$ is odd, and we may choose $k^{\prime}$ so that it does not divide $n$. (Indeed, if $k^{\prime}$ divides $n$, then $n=5 k^{\prime}$ or $7 k^{\prime}$, but there are at least three possibilities for $k^{\prime}$ since we are assuming that $n>100$ (see, e.g., [42]).) Therefore, $(n, n-k)=1$ as required.

Let $y=[A, B] \in \mathrm{GL}_{n}(2)$, where $A \in \mathrm{GL}_{k}(2)$ and $B \in \mathrm{GL}_{n-k}(2)$ are Singer cycles, so $|A|=2^{k}-1$ and $|B|=2^{n-k}-1$. Let $d$ be the largest divisor of $2^{n-k}-1$ that is relatively prime to $2^{i}-1$ for all $1 \leq i<n-k$. Note that every prime divisor of $d$ is a primitive prime divisor of $2^{n-k}-1$ and is therefore congruent to 1 modulo $n-k$. Since $n-k \geq 5$ is odd, [24, Lemma 2.1] implies that $d>2(n-k)+1$. In addition, since $k<n-k$, it follows that $d$ and $2^{k}-1$ are coprime and that some power of $y$ has order $d$. Moreover, since $n-k$ is odd, it follows that a primitive prime divisor of $2^{n-k}-1$ is also a primitive prime divisor of $4^{n-k}-1$. Now, if $d$ is a prime, then a power of $y$ has order a primitive prime divisor $r$ of $4^{n-k}-1$, with $r>2(n-k)+1$. Also, if $d$ is composite, then some power of $y$ has order a product of two (not necessarily distinct) primitive prime divisors of $4^{n-k}-1$. Consequently, Theorem 2.12 applies, and we obtain a short list of possible subgroups $H \in \mathcal{M}$, where $\mathcal{M}$ is the set of maximal subgroups of $G$ that contain $g s$ (using the Shintani correspondence, we choose $s \in G_{0}$ so that $(g s)^{2}$ is $X$-conjugate to $y$ ):

(i) $H$ is a parabolic subgroup of type $P_{k}$ or $P_{n-k}$; there is exactly one subgroup of each type in $\mathcal{M}$;

(ii) $H$ is a $\mathcal{C}_{8}$-subgroup of type $\mathrm{GU}_{n}(2)$ or $\operatorname{Sp}_{n}(4)$;

(iii) $H$ is a subfield subgroup of type $\mathrm{GL}_{n}(2)$; by Proposition 2.16 , there are at most $\left|C_{\mathrm{PGL}_{n}(2)}(y)\right|=\left(2^{n-k}-1\right)\left(2^{k}-1\right)$ subgroups of this type in $\mathcal{M}$.

In fact, it is easy to see that there are no $\mathcal{C}_{8}$-subgroups in $\mathcal{M}$. Indeed, if $H$ is of type $\mathrm{GU}_{n}(2)$, then $|H|$ is not divisible by a primitive prime divisor of $2^{n-k}-1$. Similarly, we can eliminate subgroups of type $\operatorname{Sp}_{n}(4)$ since $y$ is irreducible on an odd-dimensional subspace of $V$. We deduce that if $z \in G$ has prime order, then

$$
\alpha(z)<4^{1-k}+\left(2^{n-k}-1\right)\left(2^{k}-1\right) f(n, 4) \leq 4^{1-n / 4}+2^{n} f(n, 4)<1 / 2
$$

for all $n \geq 8$, where $f(n, 4)$ is defined as in (15). Finally, if $n=7$, then $k=3$ and the first inequality yields $\alpha(z)<1 / 2$ as required. 
To complete the proof of Proposition 4.4, it remains to show that $u(G) \rightarrow$ $\infty$ as $|G| \rightarrow \infty$. If $q>49$, then the bound in (16) implies that $\alpha(z)<q^{-1 / 4}$ for all $n \geq 5$, so we may assume that $q$ (and therefore $e$ ) is bounded and that $n$ tends to infinity.

Assume that $n$ is large, and let $k$ be an integer such that $n / 4<k<$ $n / 2, n-k$ is odd, and $(n, k)=(n-k, e)=1$. (Note that $k$ exists since $e$ is bounded.) Set $y=[A, B] \in \operatorname{PGL}_{n}\left(q_{0}\right)$, where $A \in \mathrm{GL}_{k}\left(q_{0}\right)$ and $B \in$ $\mathrm{GL}_{n-k}\left(q_{0}\right)$ are irreducible. (Take suitable powers of Singer cycles so that $\operatorname{det}(y)=\lambda^{\alpha}$.) Choose $s \in G_{0}$ such that $(g s)^{e}$ is $X$-conjugate to $y$.

There is a suitable power of $(g s)^{e}$, say, $x=(g s)^{m e}$, such that $x$ has order $r$, where $r$ is a primitive prime divisor of $q_{0}^{n-k}-1$. Moreover, since $(n-k, e)=1$, it follows that $r$ is a primitive prime divisor of $q^{n-k}-1$. Now [24, Lemma 2.1] implies that either $r>2(n-k)+1$ or some other power of $(g s)^{e}$ has order $r^{\prime}$, with $r^{\prime}$ a product of two (not necessarily distinct) primitive prime divisors of $q^{n-k}-1$. In particular, by combining Theorem 2.12 and Corollary 2.15, we see that the only possibilities for $H \in \mathcal{M}$ are the following.

(i) A maximal parabolic subgroup of type $P_{k}$ or $P_{n-k}$; there is exactly one subgroup of each type in $\mathcal{M}$.

(ii) $\mathrm{A} \mathcal{C}_{8}$-subgroup of type $\operatorname{Sp}_{n}(q), O_{n}^{\epsilon}(q)$, or $\mathrm{GU}_{n}\left(q^{1 / 2}\right)$.

(iii) A subfield subgroup of type $\mathrm{GL}_{n}\left(q_{1}\right)$, where $q=q_{1}^{a}$ for some prime divisor $a$ of $e$.

Since $r$ is a primitive prime divisor of $q^{n-k}-1$, and we have chosen $k$ so that $n-k$ is odd, it follows that $x$ does not belong to a $\mathcal{C}_{8}$-subgroup of type $\operatorname{Sp}_{n}(q)$ or $O_{n}^{\epsilon}(q)$, so there are no such subgroups in $\mathcal{M}$. Next, we observe that there are no $\mathcal{C}_{8}$-subgroups of type $\mathrm{GU}_{n}\left(q^{1 / 2}\right)$ in $\mathcal{M}$. Since $r$ is a primitive prime divisor of $q^{n-k}-1$, it follows that $r$ does not divide $\left|\mathrm{PGU}_{n}\left(q^{1 / 2}\right)\right|$. Indeed, suppose that $r$ divides $q^{j / 2}-(-1)^{j}$ for some $2 \leq j \leq n$. If $j$ is even, then $r$ divides $q^{j / 2}-1$, which is absurd since $r$ is a primitive prime divisor of $q^{n-k}-1$. Similarly, if $j$ is odd, then Lemma 4.3 implies that $r$ divides $\left(q^{j / 2}+1, q^{n-k}-1\right)=(2, q-1)$, which once again is a contradiction.

Finally, note that for each prime divisor $a$ of $e$, there are at most $\left|C_{\mathrm{PGL}_{n}\left(q_{0}\right)}(y)\right|=\left(q_{0}^{n-k}-1\right)\left(q_{0}^{k}-1\right) /\left(q_{0}-1\right)<q^{n / e}$ subfield subgroups of type $\mathrm{GL}_{n}\left(q_{1}\right)$ (where $q=q_{1}^{a}$ ) in $\mathcal{M}$ (see Proposition 2.16(i)). Therefore, by applying Theorem 2.7, we conclude that if $n$ is sufficiently large and $z \in G$ has prime order, then

$$
\alpha(z)<2 q^{-k}+2 q^{k-n}+(\log (e)+1) q^{n / e} \cdot f(n, q) .
$$


Now $f(n, q)<q^{3-n-2 / n}$ and $q$ is bounded; hence, $\alpha(z) \rightarrow 0$ as $n \rightarrow \infty$, and the result follows.

To complete the proof of Theorem 4.1, it remains to deal with the smalldimensional groups with $n \leq 4$.

Proposition 4.6. Theorem 4.1 holds when $n=4$.

Proof. In view of Proposition 2.17, we may assume that $q \geq 16$. Take $y=[A, \mu] \in \mathrm{PGL}_{4}\left(q_{0}\right)$, where $A \in \mathrm{GL}_{3}\left(q_{0}\right)$ is irreducible of order $q_{0}^{3}-1$ and $\mu=\lambda^{\alpha} / \operatorname{det}(A)$. By Lemma 4.2, there exists $s \in G_{0}$ such that $(g s)^{e}$ is $X$ conjugate to $y$. Note that a suitable power of $(g s)^{e}$, say, $x=(g s)^{m e}$, has order $r$, where $r$ is a primitive prime divisor of $q_{0}^{3}-1$.

Let $\mathcal{M}$ be the set of maximal subgroups of $G$ containing $g s$, and let $H \in \mathcal{M}$. We claim that one of the following holds:

(i) $\quad H$ is a parabolic subgroup of type $P_{1}$ or $P_{3}$; there is a unique subgroup of each type in $\mathcal{M}$;

(ii) $e \equiv 0(\bmod 3)$, and $H$ is a $\mathcal{C}_{2}$-subgroup of type $\mathrm{GL}_{1}(q)$ \& $S_{4}$; there is a unique such subgroup in $\mathcal{M}$;

(iii) $H$ is a subfield subgroup of type $\mathrm{GL}_{4}\left(q_{1}\right)$, where $q=q_{1}^{a}$ for some prime divisor $a$ of $e$; for each $q_{1}$, there are at most $\left|C_{\mathrm{PGL}_{4}\left(q_{0}\right)}(y)\right|=q_{0}^{3}-1$ such subgroups in $\mathcal{M}$.

By Corollary 2.15, the only reducible subgroups in $\mathcal{M}$ are parabolic of type $P_{1}$ or $P_{3}$; there are unique such subgroups because $y$ fixes a unique $i$-dimensional subspace of the natural $\mathrm{PGL}_{4}\left(q_{0}\right)$-module, for $i=1,3$. Part (iii) on subfield subgroups follows in the usual way from Proposition 2.16(i).

Next, suppose that $H \in \mathcal{M}$ is a $\mathcal{C}_{2}$-subgroup. If $(3, e)=1$, then $r$ is a primitive prime divisor of $q^{3}-1$, and thus $\mathcal{C}_{2}$-subgroups are ruled out by the main theorem of [25]. Now assume that $e$ is a multiple of 3, so the eigenvalues of $(g s)^{e}$ are contained in $\mathbb{F}_{q}$. Note that $r \equiv 1(\bmod 3)$, and thus $r \geq 7$. In particular, if $(g s)^{e}$ stabilizes a decomposition $V=V_{1} \oplus V_{2} \oplus V_{3} \oplus V_{4}$, then $x$ must fix each $V_{i}$, so the $V_{i}$ are simply the eigenspaces of $x$, and we conclude that there is a unique $\mathcal{C}_{2}$-subgroup of type $\mathrm{GL}_{1}(q)$ 2 $S_{4}$ in $\mathcal{M}$.

Now assume that $g s$ stabilizes a decomposition $V=V_{1} \oplus V_{2}$ with $\operatorname{dim} V_{i}=2$. By the same reasoning, $x$ must fix $V_{1}$ and $V_{2}$. Without loss of generality, let us assume that the restriction of $x$ to $V_{1}$ has eigenvalues 1 and $\xi$, while $x$ restricted to $V_{2}$ has eigenvalues $\xi^{q_{0}}$ and $\xi^{q_{0}^{2}}$. As observed in the proof of Lemma 4.5, since $g s$ commutes with $x$, it sends 1-eigenvectors 
of $x$ to 1-eigenvectors of $(g s)^{e}$, and thus $g s$ fixes $V_{1}$. This is a contradiction because we have already observed that $g s$ does not fix a 2-dimensional subspace of $V$. Therefore, there are no $\mathcal{C}_{2}$-subgroups of type $\mathrm{GL}_{2}(q)$ i $S_{2}$ in $\mathcal{M}$.

The $\mathcal{C}_{4}, \mathcal{C}_{6}$, and $\mathcal{C}_{7}$ families are empty, and the same is true for the $\mathcal{C}_{9}$ family since $q \neq p$ (see [5, Table 7.9]). We can quickly eliminate $\mathcal{C}_{3}$-subgroups of type $\mathrm{GL}_{2}\left(q^{2}\right)$ because the eigenvalues of $(g s)^{e}$ do not consist of two pairs, with elements in each pair having the same multiplicative order (as elements of $\mathbb{F}_{q^{3}}^{*}$ ). Therefore, it remains to deal with the subgroups in $\mathcal{C}_{8}$.

Suppose that $H \in \mathcal{M}$ is a $\mathcal{C}_{8}$-subgroup of type $\operatorname{GU}_{4}\left(q^{1 / 2}\right)$, so $q=q_{0}^{e}$ is an even power of $p$, and we may write $q^{1 / 2}=q_{0}^{f}$. Let $\mathcal{E}=\left\{\mu, \omega, \omega^{q_{0}}, \omega^{q_{0}^{2}}\right\}$ be the set of eigenvalues of $(g s)^{e}$, where $\mathbb{F}_{q_{0}^{3}}^{*}=\langle\omega\rangle$, and let $T$ be a maximal torus of $H$ containing $(g s)^{e}$. The conjugacy classes of maximal tori of $\mathrm{GU}_{4}\left(q_{0}^{f}\right)$ are parameterized by the conjugacy classes in $S_{4}$, which is the Weyl group of the corresponding root system of type $A_{3}$. For example, the class of transpositions in $S_{4}$ corresponds to a class of maximal tori with structure $Z_{i} \times Z_{j} \times Z_{j}$, where $i=q_{0}^{2 f}-1$ and $j=q_{0}^{f}+1$. The eigenvalues of elements in such a torus are of the form $\left\{a, a^{q_{0}^{f}}, b, c\right\}$, where $a$ has multiplicative order dividing $q_{0}^{2 f}-1$ and $b$ and $c$ have multiplicative order dividing $q_{0}^{f}+1$. In particular, the two eigenvalues corresponding to the $q_{0}^{2 f}-1$ factor have the same multiplicative order. Now three of the four eigenvalues of $(g s)^{e}$ have multiplicative order $q_{0}^{3}-1$, so these must correspond to cyclic factors of $T$ of order $q_{0}^{4 f}-1$ or $q_{0}^{2 f}-1$. Therefore, the only possible tori are of the form $q_{0}^{4 f}-1$ or $\left(q_{0}^{2 f}-1\right) \times\left(q_{0}^{2 f}-1\right)$, but the eigenvalues of any element in such a torus either all have the same multiplicative order or occur in pairs having the same order. This contradiction rules out $\mathcal{C}_{8}$-subgroups of type $\mathrm{GU}_{4}\left(q^{1 / 2}\right)$ in $\mathcal{M}$.

Next, assume that $H \in \mathcal{M}$ is a $\mathcal{C}_{8}$-subgroup of type $\operatorname{Sp}_{4}(q)$. Now $(g s)^{e\left(q_{0}-1\right)}$ has eigenvalues $\left\{1, \xi, \xi^{q_{0}}, \xi^{q_{0}^{2}}\right\}$ for some $\xi \in \mathbb{F}_{q_{0}^{3}}$ of multiplicative order $q_{0}^{2}+q_{0}+1$, but this is a contradiction since the nontrivial eigenvalues of semisimple elements in $H \cap G_{0}$ occur in pairs with the same multiplicative order. The same argument also rules out $\mathcal{C}_{8}$-subgroups of type $O_{4}^{\epsilon}(q)$.

Putting all this together, and applying Corollary 2.9 and Theorem 2.7, we deduce that if $z \in G$ has prime order, then

$$
\alpha(z)<2 q^{-1}+2 q^{-3}+\left(1+(1+\log (e))\left(q_{0}^{3}-1\right)\right) \cdot f(4, q)
$$


with $f(4, q)$ defined in (15). If $e \geq 3$, then this bound implies that $\alpha(z)<$ $1 / 2$. (Recall that we are assuming that $q \geq 16$.) Now, if $e=2$, then by Proposition 2.16(ii) we may replace the term $\left(1+(1+\log (e))\left(q_{0}^{3}-1\right)\right)$ in the above bound by $e^{2}=4$, and subsequently we deduce that $\alpha(z)<0.187$ for all $q_{0} \geq 4$. Finally, the reader can check that the above bounds imply that $\alpha(z)<q^{-1 / 4}$ for all $q>27$; hence, $u(G) \rightarrow \infty$ as $q \rightarrow \infty$.

Proposition 4.7. Theorem 4.1 holds when $n=3$.

Proof. If $q \leq 16$, then the result follows from Proposition 2.17, so let us assume that $q \geq 25$. In fact, if $q \leq 49$, then the desired result can be verified using Magma, so we will assume that $q>49$. Set $y=[A, \mu] \in \mathrm{PGL}_{3}\left(q_{0}\right)$, where $A \in \mathrm{GL}_{2}\left(q_{0}\right)$ is irreducible of order $q_{0}^{2}-1$ and where $\mu=\lambda^{\alpha} / \operatorname{det}(A)$, and fix $s \in G_{0}$ such that $(g s)^{e}$ is $X$-conjugate to $y$. As before, let $\mathcal{M}$ be the set of maximal subgroups of $G$ containing $g s$. We claim that if $H \in \mathcal{M}$, then one of the following holds:

(i) $H$ is a parabolic subgroup of type $P_{1}$ or $P_{2} ; \mathcal{M}$ contains a unique subgroup of each type;

(ii) $e$ is even, and $H$ is a $\mathcal{C}_{2}$-subgroup of type $\mathrm{GL}_{1}(q)$ \& $S_{3}$; there is a unique such subgroup in $\mathcal{M}$ if $q_{0} \neq 2$, and there are at most three when $q_{0}=2$;

(iii) $H$ is a subfield subgroup of type $\mathrm{GL}_{3}\left(q_{1}\right)$, where $q=q_{1}^{a}$ for some prime divisor $a$ of $e$; for each $q_{1}$, there are at most $\left|C_{\mathrm{PGL}_{3}\left(q_{0}\right)}(y)\right|=q_{0}^{2}-1$ such subgroups in $\mathcal{M}$;

(iv) $H$ is a $\mathcal{C}_{8}$-subgroup of type $\mathrm{GU}_{3}\left(q^{1 / 2}\right)$ or $O_{3}(q)$. In both cases, there are at most $q_{0}^{2}-1$ such subgroups in $\mathcal{M}$.

The argument here is very similar to the one given in the proof of Proposition 4.6, so we will give details only for $\mathcal{C}_{2}$ and $\mathcal{C}_{9}$-subgroups. (Note that in items (iii) and (iv), we use Proposition 2.16(i) to bound the number of subgroups of the given type in $\mathcal{M}$.)

Suppose that $H \in \mathcal{M}$ is a $\mathcal{C}_{2}$-subgroup of type $\mathrm{GL}_{1}(q)$ 2 $S_{3}$; say, $H$ preserves the decomposition $V=V_{1} \oplus V_{2} \oplus V_{3}$. If $e$ is odd, then $A \in \mathrm{GL}_{2}(q)$ is irreducible, so $(g s)^{e}$ must swap two of the $V_{i}$. Therefore, $\left|(g s)^{e}\right|$ divides $2(q-1)$, but this is a contradiction since Lemma 4.3 implies that $\left(q_{0}^{2}-\right.$ $\left.1,2\left(q_{0}^{e}-1\right)\right) \leq 2 q_{0}-2<q_{0}^{2}-1$. Now assume that $e$ is even. If $q_{0}=2$, then Proposition 2.16(i) implies that there are at most $\left|C_{\mathrm{PGL}_{3}\left(q_{0}\right)}(y)\right|=3$ subgroups of type $\mathrm{GL}_{1}(q)$ ? $S_{3}$ in $\mathcal{M}$, so let us assume that $q_{0}>2$. Since $e$ is even, the eigenvalues of $(g s)^{e}$ are in $\mathbb{F}_{q}$. Now $\left|(g s)^{e}\right|=q_{0}^{2}-1>3$, so either 
$(g s)^{2 e}$ or $(g s)^{3 e}$ fixes each $V_{i}$; hence, the $V_{i}$ are simply the (distinct) $\mathbb{F}_{q^{-}}$ eigenspaces of this element. It follows that there is a unique such subgroup in $\mathcal{M}$.

Finally, suppose that $H \in \mathcal{M}$ is a $\mathcal{C}_{9}$-subgroup. Since $q \neq p$, the only possibility is $q=p^{2}$ (so $q_{0}=p$ and $\left.e=2\right)$, with $p \equiv 2,3(\bmod 5)(p \neq 3)$, and $H$ has socle $A_{6}$ (see [5, Table 7.4]). We are assuming that $q>49$, so the congruence condition implies that $q_{0} \geq 13$. By considering the eigenvalues of $(g s)^{2}$, we deduce that $\left|(g s)^{2}\right| \geq 2 q_{0}+2 \geq 28$, but no element in $\operatorname{Aut}\left(A_{6}\right)$ has order greater than 10 , so there are no $\mathcal{C}_{9}$-subgroups in $\mathcal{M}$.

Let $z \in G$ be an element of prime order. By applying Theorem 2.7 and Lemma 2.10, we deduce that

$$
\begin{aligned}
\alpha(z)< & 2 q^{-1}+2 q^{-2}+\frac{(\log (e)+1)\left(q_{0}^{2}-1\right)+3 d-3}{q^{2}+q+1} \\
& +\frac{2(3, q-1)\left(q_{0}^{2}-1\right)(d-1)}{q^{1 / 2}(q+1)}
\end{aligned}
$$

where $d=(2, e)$. In particular, if $e \geq 3$, then $\alpha(z)<1 / 2$ for all $q>49$. Now assume that $e=2$. By applying Proposition 2.16(ii), we have

$$
\alpha(z)<2 q^{-1}+2 q^{-2}+\frac{q+2}{q^{2}+q+1}+\frac{(3, q-1)(q+3)}{q^{1 / 2}(q+1)},
$$

which is sufficient for all $q>49$. In addition, we observe that $\alpha(z)<q^{-1 / 4}$ for all $q>121$, whence $\alpha(z) \rightarrow 0$ as $q \rightarrow \infty$.

Proposition 4.8. Theorem 4.1 holds when $n=2$.

Proof. Here we may assume that $q \geq 169$ (see Proposition 2.17). Let $y \in$ $\operatorname{PGL}_{2}\left(q_{0}\right)$ be an irreducible element with determinant $\lambda^{\alpha}$, and note that $|y| \geq\left(q_{0}+1\right) /\left(2, q_{0}-1\right)$. By Lemma 4.2 , there exists $s \in G_{0}$ such that $(g s)^{e}$ and $y$ are $X$-conjugate. As usual, let $\mathcal{M}$ be the set of maximal subgroups of $G$ containing $g s$. We claim that if $H \in \mathcal{M}$, then one of the following holds:

(i) $\quad H$ is a $\mathcal{C}_{2}$-subgroup of type $\mathrm{GL}_{1}(q) 2 S_{2}$; either $e$ is even, $q_{0} \neq 3$, and there is a unique such subgroup in $\mathcal{M}$, or $q_{0}=3$ and there are at most four;

(ii) $\quad H$ is a $\mathcal{C}_{3}$-subgroup of type $\mathrm{GL}_{1}\left(q^{2}\right)$, and there is at most one subgroup of this type in $\mathcal{M}$;

(iii) $H$ is a subfield subgroup of type $\mathrm{GL}_{2}\left(q_{1}\right)$, where $q=q_{1}^{a}$ for some prime divisor $a$ of $e$. There are at most $q_{0}+1$ such subgroups in $\mathcal{M}$. 
To see this, first observe that there are no reducible subgroups in $\mathcal{M}$ since $y$ is irreducible, while the $\mathcal{C}_{4}, \mathcal{C}_{6}, \mathcal{C}_{7}$, and $\mathcal{C}_{8}$ families are empty. If $H$ is a $\mathcal{C}_{3}$-subgroup of type $\operatorname{GL}_{1}\left(q^{2}\right)$, then $\operatorname{fpr}(y, G / H)=2 /(q(q-1))$ and $[G: H]=q(q-1) / 2$, so $y$ (and thus $g s$ ) is contained in a unique $\mathcal{C}_{3}$-subgroup. As usual, the claim for subfield subgroups follows from Proposition 2.16(i) since $\left|C_{\mathrm{PGL}_{2}\left(q_{0}\right)}(y)\right|=q_{0}+1$.

Suppose that $H \in \mathcal{M}$ is a $\mathcal{C}_{9}$-subgroup. Here the only possibility is $q=p^{2}$ (so $q_{0}=p$ and $\left.e=2\right)$, with $p \equiv \pm 3(\bmod 10)$, and $H$ has socle $A_{5}$. As previously stated, we may assume that $q_{0} \geq 13$, and thus $\left|(g s)^{e}\right| \geq 7$. However, no element in $S_{5}$ has order greater than 6 , so there are no such subgroups in $\mathcal{M}$.

Finally, suppose that $H$ is a $\mathcal{C}_{2}$-subgroup of type $\mathrm{GL}_{1}(q)$ ₹ $S_{2}$; say, $H$ preserves the decomposition $V=V_{1} \oplus V_{2}$. If $q_{0}=3$, then Proposition 2.16(i) implies that there are at most $\left|C_{\mathrm{PGL}_{2}\left(q_{0}\right)}(y)\right|=4$ subgroups of this type in $\mathcal{M}$, so let us assume that $q_{0} \neq 3$. If $e$ is even, then $(g s)^{2 e}$ fixes each $V_{i}$, so $V_{1}$ and $V_{2}$ are the eigenspaces of $(g s)^{2 e}$ (note that the eigenvalues are distinct since $q_{0} \neq 3$ ), and thus $\mathcal{M}$ contains a unique subgroup of this type. Now assume that $e$ is odd. Here $(g s)^{e}$ must swap $V_{1}$ and $V_{2}$, so $\left|(g s)^{e}\right|$ divides $2(q-1)$. This is a contradiction if $q_{0}$ is even since $\left|(g s)^{e}\right|=q_{0}+1$. Now assume that $q_{0}$ is odd, so $\left|(g s)^{e}\right| \geq\left(q_{0}+1\right) / 2$. The previous divisibility condition implies that $q_{0}$ is a Mersenne prime. Therefore, $2(q-1) \equiv 4(\bmod 8)$, and thus the same divisibility criterion implies that $q_{0}=7$. Here a maximal $\mathcal{C}_{2}$-subgroup of $\mathrm{PGL}_{2}(q)$ is a dihedral group of order $2\left(7^{e}-1\right)$. In particular, since $7^{e}-1$ is indivisible by 4 (recall that $e$ is odd), and the exponent of the dihedral group is $7^{e}-1$, it follows that $(g s)^{e}$ is not contained in $H$.

Let $z \in G$ be an element of prime order, and suppose that $H \in \mathcal{M}$. According to [35, Theorem 1], either $\operatorname{fpr}(z, G / H) \leq 2 /(q+1)$, or $H$ is of type $\mathrm{GL}_{2}\left(q^{1 / 2}\right)$ and

$$
\operatorname{fpr}(z, G / H) \leq \frac{2+q^{1 / 2}\left(q^{1 / 2}+1\right)}{q^{1 / 2}(q+1)}
$$

Therefore,

$$
\alpha(z) \leq \frac{2\left(q_{0}+1\right)(\log (e)+2-d)+10}{q+1}+(d-1)\left(q_{0}+1\right) \cdot\left(\frac{2+q^{1 / 2}\left(q^{1 / 2}+1\right)}{q^{1 / 2}(q+1)}\right)
$$

for all $z \in G$ of prime order, where $d=(2, e)$. In particular, if $e \geq 3$ and $q \geq 169$, then $\alpha(z)<1 / 2$ as required. Finally, if $e=2\left(\right.$ and $q \geq 169$ so $\left.q_{0} \neq 3\right)$, 
then by applying Proposition 2.16(ii), we deduce that

$$
\alpha(z) \leq \frac{4}{q+1}+2\left(\frac{2+q^{1 / 2}\left(q^{1 / 2}+1\right)}{q^{1 / 2}(q+1)}\right)<1 / 2 .
$$

In addition, the above bounds imply that $\alpha(z)<q^{-1 / 7}$ for all $q \geq 169$, whence $u(G) \rightarrow \infty$ as $q \rightarrow \infty$.

This completes the proof of Theorem 4.1.

\section{§5. Graph-field automorphisms}

Here $n \geq 3$, and $G=\left\langle G_{0}, g\right\rangle$, with $g=\iota \sigma x$, where $\iota$ is the inverse-transpose graph automorphism of $G_{0}, \sigma$ is a standard field automorphism (of order $e>1$ ), and $x \in \mathrm{PGL}_{n}(q)$. In particular, $q=q_{0}^{e}$, and we note that $\iota \sigma=\sigma \iota$. As before, we may replace $x$ by $\delta=\left[\lambda, I_{n-1}\right]$ for some $\lambda \in \mathbb{F}_{q}^{*}$. The idea is to modify the approach used in Section 4 , based on Shintani descent.

Let $K$ be the algebraic closure of $\mathbb{F}_{q}$, and set $X=\operatorname{PSL}_{n}(K)$. We may view $\iota \sigma$ as a Frobenius morphism of $X$. As stated in Lemma 2.13, the associated Shintani map provides a bijective correspondence between the set of $X_{(\iota \sigma)^{e^{-}}}$ classes in the coset $\iota \sigma X_{(\iota \sigma)^{e}}$ and the set of $X_{\iota \sigma}$-classes in $X_{\iota \sigma}=\operatorname{PGU}_{n}\left(q_{0}\right)$. If $e$ is even, then $X_{(\iota \sigma)^{e}}=X_{\sigma^{e}}=\mathrm{PGL}_{n}(q)$, and we can proceed as in Section 4. However, if $e$ is odd, then we cannot realize $\operatorname{PGL}_{n}(q)$ as the set of fixed points in $X$ of some power of $\iota \sigma$. Indeed, $X_{(\iota \sigma)^{2 m+1}}=X_{\iota \sigma^{2 m+1}}=$ $\operatorname{PGU}_{n}\left(q_{0}^{2 m+1}\right)$ for all $m$. Therefore, a modified approach is required to handle this case.

The main result of this section is the following.

Theorem 5.1. Let $G_{0}=\operatorname{PSL}_{n}(q)$, and let $G=\left\langle G_{0}, g\right\rangle$, where $n \geq 3$ and $g=\iota \sigma x$ is the product of the inverse-transpose graph automorphism $\iota, a$ standard field automorphism $\sigma$ of order $e>1$ and $x \in \mathrm{PGL}_{n}(q)$. If we assume that $G \neq \mathrm{PSL}_{3}(4) .2_{1}$, then there exists $s \in G_{0}$ such that

$$
\sum_{H \in \mathcal{M}(g s)} \operatorname{fpr}(z, G / H)<1 / 2
$$

for all $z \in G$ of prime order. In particular, $u(G) \geq 2$ for all $G$. Moreover, $u(G)$ is bounded as $|G| \rightarrow \infty$ if and only if $q$ is bounded and ne is odd.

REMARK 5.2. The excluded case $G=\operatorname{PSL}_{3}(4) .2_{1}$ is a genuine exception to the bound in (17), but it is easy to check that $u(G) \geq 2$ (see Proposition 2.18). 
As previously remarked, the analysis here depends on the parity of $e$ (where $e$ is the order of the field automorphism $\sigma$ involved in $g$ ).

\section{1. $\sigma$ has even order}

Let $f$ be the Shintani map from the set of $\mathrm{PGL}_{n}(q)$-classes in the coset $\iota \sigma \mathrm{PGL}_{n}(q)$ to the set of $\mathrm{PGU}_{n}\left(q_{0}\right)$-classes in $\mathrm{PGU}_{n}\left(q_{0}\right)$. We start with an analogue of Lemma 4.2 .

LEMMA 5.3. With the notation above, let $\alpha=-(q-1) /\left(q_{0}+1\right)$, and suppose that $y \in \mathrm{PGU}_{n}\left(q_{0}\right)$ has determinant $\lambda^{\alpha}$. Then there exists $s \in G_{0}$ such that $f(g s)$ is $\mathrm{PGU}_{n}\left(q_{0}\right)$-conjugate to $y$.

Proof. Since $f$ is a bijection, the $\mathrm{PGU}_{n}\left(q_{0}\right)$-class of $y$ corresponds to the $\mathrm{PGL}_{n}(q)$-class of $\iota \sigma t$ for some $t \in \mathrm{PGL}_{n}(q)$. Let $\mu$ be the determinant of $t$, and fix a generator $\omega$ for $\mathbb{F}_{q}^{*}$. Now

$$
(\iota \sigma t)^{e}=t^{(\iota \sigma)^{e-1}} t^{(\iota \sigma)^{e-2}} \cdots t^{\iota \sigma} t
$$

and $\operatorname{det} t^{(\iota \sigma)^{j}}=\mu^{\left(-q_{0}\right)^{j}}$, so $f(\iota \sigma t)$ has determinant $\mu^{\alpha}$. Since $f(\iota \sigma t)$ and $y$ are $\mathrm{PGU}_{n}\left(q_{0}\right)$-conjugate, it follows that $\mu^{\alpha}=\lambda^{\alpha}$, and thus $\mu=\omega^{\left(q_{0}+1\right) j} \lambda$ for some integer $0 \leq j<\alpha$. Let $x \in \mathrm{PGL}_{n}(q)$ be an element with determinant $\omega^{-j}$. Then $(\iota \sigma t)^{x}=\iota \sigma x^{-\iota \sigma} t x$, and $x^{-\iota \sigma} t x$ has determinant $\lambda$. Therefore, $x^{-\iota \sigma} t x \in \delta G_{0}$, so there exists $s \in G_{0}$ such that $\iota \sigma x^{-\iota \sigma} t x=\iota \sigma \delta s=g s \in G$ corresponds to $y$ under the Shintani correspondence.

Proposition 5.4. Theorem 5.1 holds when $e$ is even and $n \geq 5$.

Proof. This is very similar to the proof of Proposition 4.4. Set $y=[A, B] \in$ $\mathrm{PGU}_{n}\left(q_{0}\right)$, where $A \in \mathrm{GU}_{n-2}\left(q_{0}\right)$ is a regular semisimple element of order $\left(q_{0}^{n-2}-(-1)^{n}\right)\left|\lambda^{\alpha}\right| /\left(q_{0}+1\right)$ and determinant $\lambda^{\alpha}$ (here $\alpha=-(q-1) /\left(q_{0}+1\right)$, as in Lemma 5.3), and $B \in \mathrm{GU}_{2}\left(q_{0}\right)$ is a unitary transvection. Note that if $n$ is odd, then $A$ is irreducible over $\mathbb{F}_{q_{0}^{2}}$, while $A$ splits into two irreducible blocks of dimension $n / 2-1$ when $n$ is even. Since $A$ (and therefore $y$ ) has determinant $\lambda^{\alpha}$, a combination of Lemmas 2.13 and 5.3 implies that there exists $s \in G_{0}$ such that $(g s)^{e}$ and $y$ are $X$-conjugate. If $(n, q)=(6,4)$, then Proposition 2.17 applies, so we may assume that $(n, q) \neq(6,4)$.

To determine the subgroups in $\mathcal{M}=\mathcal{M}(g s)$, we proceed as in the proof of Lemma 4.5. First, observe that a suitable power of $(g s)^{e}$ is a transvection; this immediately eliminates the subgroups in the $\mathcal{C}_{3}, \mathcal{C}_{4}, \mathcal{C}_{6}, \mathcal{C}_{7}$, and $\mathcal{C}_{9}$ collections, and we can also rule out $\mathcal{C}_{8}$-subgroups of type $O_{n}^{\epsilon}(q)$ for the same reason. 
Next, let us turn to the $\mathcal{C}_{1}$-subgroups in $\mathcal{M}$. First, note that $y$ is contained in a unique maximal parabolic subgroup of $\mathrm{PGU}_{n}\left(q_{0}\right)$ of type $P_{1}$ and also a unique subgroup of type $\mathrm{GU}_{2}\left(q_{0}\right) \perp \mathrm{GU}_{n-2}\left(q_{0}\right)$ (the stabilizer of a nondegenerate 2-space). In addition, if $n$ is even, then $y$ is contained in exactly two subgroups of type $P_{n / 2-1}$ and two of type $P_{n / 2}$. By applying Corollary 2.15, we deduce that the $\mathcal{C}_{1}$-subgroups in $\mathcal{M}$ are as follows: one each of type $P_{1, n-1}$ and $\mathrm{GL}_{2}(q) \times \mathrm{GL}_{n-2}(q)$, in addition to two each of type $P_{n / 2-1, n / 2+1}$ and $P_{n / 2}$ when $n$ is even. (Recall that $P_{i, j}$ denotes the $G$ stabilizer of a pair of subspaces $U \subset W$ of $V$, where $\operatorname{dim} U=i, \operatorname{dim} W=j$, and $i+j=n$; such subgroups are maximal in $G$ whenever $G \not \leq \operatorname{P\Gamma L}_{n}(q)$.)

Let us explain in more detail how Corollary 2.15 applies in this situation. Let $Y$ be a parabolic subgroup of type $P_{1, n-1}$ of the algebraic group $X=\operatorname{PSL}_{n}\left(\overline{\mathbb{F}}_{q}\right)$. Recall that $e$ is even, so we have $(\iota \sigma)^{e}=\sigma^{e}$. Now Corollary 2.15 implies that the number of $X_{\sigma^{e}}=\mathrm{PGL}_{n}(q)$-conjugates of $Y_{\sigma^{e}}$ (a type $P_{1, n-1}$ parabolic subgroup of $\left.\operatorname{PGL}_{n}(q)\right)$ normalized by $\iota \sigma s$ is equal to the number of $X_{\iota \sigma}=\mathrm{PGU}_{n}\left(q_{0}\right)$-conjugates of $Y_{\iota \sigma}$ (a type $P_{1}$ parabolic subgroup of $\left.\mathrm{PGU}_{n}\left(q_{0}\right)\right)$ containing $f(\iota \sigma s)$. We have already observed that $f(\iota \sigma s)$ is contained in a unique $P_{1}$ parabolic subgroup of $\mathrm{PGU}_{n}\left(q_{0}\right)$, so there is only one subgroup of type $P_{1, n-1}$ in $\mathcal{M}$. Similarly, suppose that $Y$ is a Levi subgroup of $X$ of type $A_{1} A_{n-3} T_{1}$. Then $Y_{\sigma^{e}}$ is a type $\mathrm{GL}_{2}(q) \times \mathrm{GL}_{n-2}(q)$ subgroup of $X_{\sigma^{e}}=\mathrm{PGL}_{n}(q)$, and $Y_{\iota \sigma}$ is a type $\mathrm{GU}_{2}\left(q_{0}\right) \times \mathrm{GU}_{n-2}\left(q_{0}\right)$ subgroup of $X_{\iota \sigma}=\mathrm{PGU}_{n}\left(q_{0}\right)$. Since $f(\iota \sigma s) \in \mathrm{PGU}_{n}\left(q_{0}\right)$ is contained in only one such subgroup, Corollary 2.15 implies that there is only one subgroup of $\mathrm{PGL}_{n}(q)$ of type $\mathrm{GL}_{2}(q) \times \mathrm{GL}_{n-2}(q)$ normalized by $\iota \sigma s$. The other cases are similar.

Now assume that $H \in \mathcal{M}$ is a $\mathcal{C}_{2}$-subgroup. By arguing as in the proof of Lemma 4.5, we deduce that one of the following holds:

(i) $H$ is of type $\mathrm{GL}_{2}(q) 2 S_{n / 2}$, and $e \geq(n-2) / 2$; there is at most one subgroup of this type in $\mathcal{M}$;

(ii) $H$ is of type $\operatorname{GL}_{1}(q) \imath S_{n}, q$ is even, and $e \geq n-2$; there are at most $q_{0} / 2$ subgroups of this type in $\mathcal{M}$.

Next, suppose that $H$ is a $\mathcal{C}_{5}$-subgroup of type $\operatorname{GL}_{n}\left(q_{1}\right)$, where $q=q_{1}^{r}$ for a prime $r$. (As before, we note that $r$ divides e.) If $e \geq 3$, then Proposition 2.16(i) implies that for each relevant prime $r$ there are at most $\left|C_{\mathrm{PGU}_{n}\left(q_{0}\right)}(y)\right| \leq q_{0}\left(q_{0}^{n-2}+1\right)$ distinct $\mathcal{C}_{5}$-subgroups in $\mathcal{M}$. Now assume that $e=2$, so $r=2$ and $n$ is even. We claim that there are no $\mathcal{C}_{5}$-subgroups in $\mathcal{M}$. 
To see this, first note that the set of eigenvalues of $(g s)^{e}$ is of the form

$$
\mathcal{E}=\left\{1, \omega^{k}, \omega^{-q_{0} k}, \omega^{q_{0}^{2} k}, \ldots, \omega^{\left(-q_{0}\right)^{n-3} k}\right\},
$$

where $\mathbb{F}_{q_{0}^{n-2}}^{*}=\langle\omega\rangle$ and $k=\left(q_{0}+1\right) /\left|\lambda^{\alpha}\right|\left(\right.$ with $\alpha=-(q-1) /\left(q_{0}+1\right)$ as before). Now, if $(g s)^{e}$ is contained in a $\mathcal{C}_{5}$-subgroup of type $\operatorname{GL}_{n}\left(q_{0}\right)$, then $\omega^{q_{0} k}$ is an eigenvalue of $(g s)^{e}$, so $\omega^{q_{0} k}=\omega^{\left(-q_{0}\right)^{j} k}$ for some $0 \leq j \leq n-3$, and thus $q_{0}^{n-2}-1$ divides $k\left(q_{0}-\left(-q_{0}\right)^{j}\right)$. However, if $j \leq n-4$, then $\mid k\left(q_{0}-\right.$ $\left.\left(-q_{0}\right)^{j}\right) \mid<q_{0}^{n-2}-1$, which is a contradiction. Similarly, if $j=n-3$ and $\left|\lambda^{\alpha}\right| \neq 1$, then $k \leq\left(q_{0}+1\right) / 2$ and $\left|k\left(q_{0}-\left(-q_{0}\right)^{j}\right)\right|<q_{0}^{n-2}-1$, whereas if $\left|\lambda^{\alpha}\right|=1$, then $k\left(q_{0}-\left(-q_{0}\right)^{j}\right)=\left(q_{0}+1\right)\left(q_{0}+q_{0}^{n-3}\right)$ is clearly indivisible by $q_{0}^{n-2}-1$. (Recall that we may assume that $(n, q) \neq(6,4)$.) This justifies the claim.

To complete the analysis of the subgroups in $\mathcal{M}$, we may assume that $H$ is a $\mathcal{C}_{8}$-subgroup of type $\operatorname{Sp}_{n}(q)$ or $\mathrm{GU}_{n}\left(q^{1 / 2}\right)$. By Proposition 2.16(i), there are at most $\left|C_{\mathrm{PGU}_{n}\left(q_{0}\right)}(y)\right| \leq q_{0}\left(q_{0}^{n-2}+1\right)$ subgroups of type $\mathrm{GU}_{n}\left(q^{1 / 2}\right)$ in $\mathcal{M}$, but we claim that there are none of type $\operatorname{Sp}_{n}(q)$. To see this, suppose that $n$ is even, and let $\mathcal{E}$ be the set of eigenvalues of $(g s)^{e}$ as above. Now $(g s)^{e} \in \operatorname{PGSp}_{n}(q)$, and thus $(g s)^{2 e} \in \operatorname{PSp}_{n}(q)$, so $\omega^{-2 k}$ is an eigenvalue of $(g s)^{2 e}$. Therefore, $\omega^{-2 k}=\omega^{2 k\left(-q_{0}\right)^{j}}$ for some $0 \leq j \leq n-3$, so $q_{0}^{n-2}-1$ divides $2 k\left(-q_{0}\right)^{j}+2 k$, and thus $\left(q_{0}^{n-2}-1,2 k\left(q_{0}^{j}+(-1)^{j}\right)\right)=q_{0}^{n-2}-1$. However,

$$
\begin{aligned}
\left(q_{0}^{n-2}-1,2 k\left(q_{0}^{j}+(-1)^{j}\right)\right) & \leq 2 k\left(q_{0}^{n-2}-1, q_{0}^{j}+(-1)^{j}\right) \\
& \leq 2\left(q_{0}+1\right)\left(q_{0}^{n-2}-1, q_{0}^{j}+(-1)^{j}\right),
\end{aligned}
$$

and Lemma 4.3 implies that this upper bound is less than $q_{0}^{n-2}-1$, which is a contradiction. For example, if $j$ is even, then Lemma 4.3 yields

$$
\begin{aligned}
& 2\left(q_{0}+1\right)\left(q_{0}^{n-2}-1, q_{0}^{j}+(-1)^{j}\right) \\
& \quad=2\left(q_{0}+1\right)\left(q_{0}^{n-2}-1, q_{0}^{j}+1\right) \leq 2\left(q_{0}+1\right)\left(q_{0}^{(n-2, j)}+1\right),
\end{aligned}
$$

and we have $2\left(q_{0}+1\right)\left(q_{0}^{(n-2, j)}+1\right)<q_{0}^{n-2}-1$ since $(n-2, j) \leq(n-2) / 2$. We conclude that there are no $\mathcal{C}_{8}$-subgroups of type $\operatorname{Sp}_{n}(q)$ in $\mathcal{M}$.

Let $z \in G$ be an element of prime order, and define $\alpha(z)$ as in (14). By applying Theorem 2.7, we deduce that the contribution to $\alpha(z)$ from the subgroups in $\mathcal{C}_{1}$ is less than

$$
q^{-1}+q^{1-n}+2 q^{-2}+((n, 2)-1)\left(4 q^{1-n / 2}+4 q^{-n / 2}\right),
$$


while Corollary 2.9 indicates that the remaining contribution is less than

$$
\left((n, 2)-1+\frac{1}{2} q_{0}((q, 2)-1)+q_{0}\left(q_{0}^{n-2}+1\right)\left(1+\left(1-\delta_{2, e}\right)(\log (e)+1)\right)\right) \cdot f(n, q),
$$

where $f(n, q)$ is given in (15). For $n \geq 5$, it is straightforward to check that these bounds imply that $\alpha(z)<1 / 2$ unless

$$
(n, q) \in\{(8,4),(7,4),(6,4),(5,9),(5,4)\} .
$$

In addition, we note that the above bounds immediately imply that $\alpha(z) \rightarrow 0$ as $q \rightarrow \infty$ (for any $n \geq 5$ ), whence $u(G) \rightarrow \infty$ as $q \rightarrow \infty$.

The cases $(n, q)=(5,4),(6,4)$ are dealt with in Proposition 2.17. To deal with the remaining cases, it is helpful to note that if $H$ is a maximal subgroup of type $\mathrm{GU}_{n}\left(q_{0}\right)$, then $\operatorname{fpr}(z, G / H) \leq \beta(n, q)$ for all $z \in G$ of prime order, where $\beta(n, q)$ is defined as follows:

\begin{tabular}{|l|l|l|l|}
\hline$(n, q)$ & $(8,4)$ & $(7,4)$ & $(5,9)$ \\
\hline$\beta(n, q)$ & $1 / 32639$ & $1 / 8128$ & $1 / 9801$ \\
\hline
\end{tabular}

Armed with these bounds, the desired result quickly follows. For example, suppose that $(n, q)=(5,9)$. As above, the $\mathcal{C}_{1}$ contribution is less than $9^{-1}+$ $9^{-4}+2 \cdot 9^{-2}$; the only other subgroups in $\mathcal{M}$ are of type $\mathrm{GU}_{5}(3)$, and thus

$$
\alpha(z)<9^{-1}+9^{-4}+2 \cdot 9^{-2}+3\left(3^{3}+1\right) / 9801<1 / 2 .
$$

Similarly, we get $\alpha(z)<1 / 2$ when $(n, q)=(8,4)$ or $(7,4)$.

To complete the proof of Proposition 5.4, it remains to show that $u(G) \rightarrow$ $\infty$ when $q$ is bounded and $n$ tends to infinity. As in the proof of Proposition 4.4 , let $k$ be an integer such that $n / 4<k<n / 2, n-k$ is odd and $(n, k)=(n-k, e)=1$. We may assume that $n$ is large. Set $y=[A, B] \in$ $\mathrm{PGU}_{n}\left(q_{0}\right)$, where $A \in \mathrm{GU}_{n-k}\left(q_{0}\right)$ is irreducible of order a multiple of $q_{0}^{n-k}+$ 1 and $B \in \mathrm{GU}_{k}\left(q_{0}\right)$ has order a multiple of $q_{0}^{k}-(-1)^{k}$. ( $B$ is irreducible when $k$ is odd; otherwise, $B$ splits into two irreducible blocks.) Note that $\left|C_{\mathrm{PGU}_{n}\left(q_{0}\right)}(y)\right|<2 q_{0}^{n-1}$. Since $(n-k, e)=1$, some power of $y$ has order $r$, where either $r$ is a primitive prime divisor of $q^{n-k}-1$ with $r>2(n-k)+1$, or $r$ is a product of primitive prime divisors of $q^{n-k}-1$. Therefore, since $n-k$ is odd, by applying Theorem 2.12 and Corollary 2.15 we deduce that the possibilities for $H \in \mathcal{M}$ are as follows (we use Proposition 2.16 to bound the number of irreducible subgroups in $\mathcal{M}$ of a given type): 
(i) $\quad H$ is of type $\mathrm{GL}_{k}(q) \times \mathrm{GL}_{n-k}(q)$; there is a unique such subgroup in $\mathcal{M}$;

(ii) $H$ is of type $P_{k / 2, n-k / 2}$, and $n$ is odd; there are at most two such subgroups in $\mathcal{M}$;

(iii) $H$ is of type $\operatorname{GL}_{n}\left(q_{1}\right)$, where $q=q_{1}^{a}$ for some prime divisor $a$ of $e$; for a given $a$, there are less than $2 q_{0}^{n-1}$ such subgroups in $\mathcal{M}$;

(iv) $H$ is of type $\mathrm{GU}_{n}\left(q^{1 / 2}\right)$; there are less than $2 q_{0}^{n-1}$ such subgroups in $\mathcal{M}$. (Note that there are no subgroups of type $\operatorname{Sp}_{n}(q)$ or $O_{n}^{\epsilon}(q)$ in $\mathcal{M}$ since $y$ acts irreducibly on a subspace of $V$ of odd dimension $n-k>n / 2$.)

Let $z \in G$ be an element of prime order. In the usual way, we calculate that

$$
\alpha(z)<2 q^{-n / 4}+4 q^{-n / 8}+2(\log (e)+2) q_{0}^{n-1} \cdot f(n, q) ;
$$

hence, $\alpha(z) \rightarrow 0$ as $n \rightarrow \infty$, as required.

Proposition 5.5. Theorem 5.1 holds when $e$ is even and $n=4$.

Proof. If $q \leq 9$, then Proposition 2.17 applies, so we may assume that $q \geq 16$. Set $y=[A, B] \in \mathrm{PGU}_{4}\left(q_{0}\right)$, where $A \in \mathrm{GU}_{3}\left(q_{0}\right)$ is irreducible of order $q_{0}^{3}+1$ and $B \in \mathrm{GU}_{1}\left(q_{0}\right)$. As usual, let $\mathcal{M}$ denote the set of maximal subgroups of $G$ containing $g s$, and note that there is a unique reducible subgroup in $\mathcal{M}$ (of type $\mathrm{GL}_{1}(q) \times \mathrm{GL}_{3}(q)$ ). Also, since $q \neq p$, we note that $G$ has no maximal $\mathcal{C}_{9}$-subgroups (see [5, Table 7.9]).

First, assume that $e$ is divisible by 3 . Since $e$ is even, it follows that $e \geq 6$, and thus $q \geq 64$. It is easy to see that there are no $\mathcal{C}_{4^{-}}, \mathcal{C}_{6^{-}}$, or $\mathcal{C}_{7^{-}}$-subgroups in $\mathcal{M}$. In addition, we can eliminate $\mathcal{C}_{3}$-subgroups, and also $\mathcal{C}_{8}$-subgroups of type $\operatorname{Sp}_{4}(q)$ or $O_{4}^{\epsilon}(q)$, because exactly three of the eigenvalues of $y$ have the same multiplicative order. For the remaining $\mathcal{C}_{2^{-}}, \mathcal{C}_{5^{-}}$, and $\mathcal{C}_{8}$-subgroups of $G$, Proposition 2.16(i) implies that there are at most $\left|C_{\mathrm{PGU}_{4}\left(q_{0}\right)}(y)\right|=q_{0}^{3}+1$ subgroups of a given type in $\mathcal{M}$. Therefore, by applying Theorem 2.7 , we deduce that

$$
\alpha(z)<q^{-1}+q^{-3}+(4+\log (e))\left(q_{0}^{3}+1\right) \cdot f(4, q)<1 / 2
$$

for all $q$, and we also observe that $\alpha(z) \rightarrow 0$ as $q \rightarrow \infty$.

For the remainder of the proof, we will assume that $e$ is indivisible by 3 . Now $\left|(g s)^{e}\right|$ is divisible by $\left(q_{0}^{3}+1\right) /\left(q_{0}+1\right)$, so every primitive prime divisor of $q_{0}^{6}-1$ divides $\left|(g s)^{e}\right|$. Moreover, since $e$ is indivisible by 3 , such a prime is a primitive prime divisor of $q^{3}-1$. In particular, if $q_{0} \notin\{2,3,5\}$, then $[24$, Lemma 2.1] implies that some power of $(g s)^{e}$ has order $r$, where either $r$ is a primitive prime divisor of $q^{3}-1$ with $r>7$, or $r$ is a product of primitive 
prime divisors of $q^{3}-1$. Therefore, in these cases we can use Theorem 2.12 to determine the subgroups in $\mathcal{M}$.

Suppose that $q_{0} \notin\{2,3,5\}$. Since $y$ acts irreducibly on a 3 -dimensional subspace of $V$, it follows that there are no $\mathcal{C}_{8}$-subgroups of type $\operatorname{Sp}_{4}(q)$ or $O_{4}^{\epsilon}(q)$ in $\mathcal{M}$. Therefore,

$$
\alpha(z)<q^{-1}+q^{-3}+(\log (e)+2)\left(q_{0}^{3}+1\right) \cdot f(4, q),
$$

and one can check that this bound is sufficient if $e \geq 4$. Now assume that $e=2$. Here the proof of Proposition 2.16(ii) reveals that there are at most $e^{2}=4$ subgroups of type $\mathrm{GU}_{4}\left(q_{0}\right)$ in $\mathcal{M}$, and we note that there are no $\mathcal{C}_{5^{-}}$ subgroups since $\mathrm{PGL}_{4}\left(q_{0}\right)$ does not contain any elements of order $\left|(g s)^{2}\right|$. Therefore,

$$
\alpha(z)<q^{-1}+q^{-3}+4 \cdot f(4, q)<1 / 2 .
$$

To complete the proof, we may assume that $q_{0} \in\{2,3,5\}$ and that $e$ is indivisible by 3 . If $e \geq 4$, then it is easy to check that (18) applies, so we reduce to the case $q=25$. Here a power of $(g s)^{2}$ has order 7 (a primitive prime divisor of $25^{3}-1$ ), and by inspecting [25] we deduce that every irreducible subgroup $H \in \mathcal{M}$ is of type $\mathrm{GU}_{4}(3)$. Therefore, (19) holds, and the result follows.

Proposition 5.6. Theorem 5.1 holds when $e$ is even and $n=3$.

Proof. This is similar to the proof of Proposition 5.5. In view of Proposition 2.17, we may assume that $q \geq 25$. Let $y \in \mathrm{PGU}_{3}\left(q_{0}\right)$ be an irreducible element of order a multiple of $\left(q_{0}^{3}+1\right) /\left(q_{0}+1\right)$, and define $\mathcal{M}$ as before. Note that there are no reducible subgroups in $\mathcal{M}$.

If $e$ is a multiple of 3, then by arguing as in the proof of Proposition 5.5, using Lemma 2.10 and Proposition 2.16(i), noting that $\left|C_{\mathrm{PGU}_{3}\left(q_{0}\right)}(y)\right|=$ $q_{0}^{2}-q_{0}+1$, we have

$$
\alpha(z)<\left(q_{0}^{2}-q_{0}+1\right)\left(\frac{(3, q-1)}{q^{1 / 2}(q+1)}+\frac{\log (e)+3}{q^{2}+q+1}\right)<1 / 2,
$$

and the result follows. For the remainder, let us assume that $e$ is indivisible by 3 .

Suppose that $q_{0} \notin\{2,3,5\}$. Then, as in the proof of Proposition 5.5, some power of $(g s)^{e}$ has order $r$, where either $r$ is a primitive prime divisor of $q^{3}-1$ with $r>7$, or $r$ is a product of primitive prime divisors of $q^{3}-1$. 
In particular, if $H \in \mathcal{M}$, then Theorem 2.12 implies that $H$ is of type $\mathrm{GL}_{1}\left(q^{3}\right), \mathrm{GU}_{3}\left(q^{1 / 2}\right)$, or $\mathrm{GL}_{3}\left(q_{1}\right)$. Moreover, if $H \in \mathcal{M}$ is of type $\mathrm{GL}_{1}\left(q^{3}\right)$, then $H \leq C_{G}\left((g s)^{e}\right)$, so there is a unique such subgroup in $\mathcal{M}$ (because $(g s)^{e}$ is contained in a unique maximal torus of $\left.C_{G}\left((g s)^{e}\right)\right)$. Now, if $e \geq 4$, then Lemma 2.10 implies that

$$
\alpha(z)<\left(q_{0}^{2}-q_{0}+1\right)\left(\frac{2(3, q-1)}{q^{1 / 2}(q+1)}+\frac{\log (e)}{q^{2}+q+1}\right)+\frac{1}{q^{2}+q+1}<1 / 2 .
$$

Similarly, if $e=2$, then the proof of Proposition 2.16(ii) implies that $\mathcal{M}$ contains at most two subgroups of type $\mathrm{GU}_{3}\left(q^{1 / 2}\right)$, and thus

$$
\alpha(z) \leq \frac{2(3, q-1)}{q^{1 / 2}(q+1)}+\frac{1}{q^{2}+q+1}<1 / 2
$$

for all $q \geq 25$. (Note that there are no subfield subgroups in $\mathcal{M}$ when $e=2$.)

Finally, suppose that $q_{0} \in\{2,3,5\}$ and that $e$ is indivisible by 3 . If $e \geq 4$, then (20) applies, so we reduce to the case $q=25$. Here we obtain the same list of subgroups in $\mathcal{M}$ as in the previous paragraph, using the main theorem of [25] and [5, Table 7.4], so (21) holds, and the result follows.

\section{2. $\sigma$ has odd order}

As before, let $K=\overline{\mathbb{F}}_{q}$, let $X=\operatorname{PSL}_{n}(K)$, and recall that we may assume that $g=\iota \sigma \delta$, where $\delta=\left[\lambda, I_{n-1}\right]$. We claim that $g$ has order $2 e$, which implies that $g$ is $\operatorname{PGL}_{n}(q)$-conjugate to $\iota \sigma$ (see [20, Theorem 7.2]). To see this, first note that the order of $g$ is certainly a multiple of $2 e$. Now an easy calculation shows that

$$
(\iota \sigma \delta)^{2}=\sigma^{2} \delta^{-\sigma} \delta=\sigma^{2}\left[\lambda^{1-q_{0}}, I_{n-1}\right]
$$

In particular, if we set $\mu=\left[\lambda^{1-q_{0}}, I_{n-1}\right]$, then

$$
(\iota \sigma \delta)^{2 e}=\left(\sigma^{2} \mu\right)^{e}=\mu^{\sigma^{2 e-2}} \mu^{\sigma^{2 e-4}} \cdots \mu^{\sigma^{2}} \mu .
$$

However, since $e$ is odd and $\mu^{\sigma^{e}}=\mu$, this is equal to

$$
\mu^{\sigma^{e-1}} \mu^{\sigma^{e-2}} \cdots \mu^{\sigma} \mu=\left[\lambda^{\left(1-q_{0}\right)\left(q_{0}^{e-1}+q_{0}^{e-2}+\cdots+1\right)}, I_{n-1}\right]=1 .
$$

This justifies the claim. Therefore, we may assume that $g=\iota \sigma$.

Let $\gamma$ be the standard involutory graph automorphism of $X$ induced from the order 2 symmetry of the corresponding Dynkin diagram of type $A_{n-1}$. 
Set $Y=C_{X}(\gamma)$, and note that $Y=\operatorname{PSO}_{n}(K)$ or $\operatorname{PSp}_{n}(K)$ when $n$ is odd or even, respectively (see [21, Theorem 1.15.2(d)]). In particular, $Y$ is a simple algebraic group, and we can use the tools of Shintani descent. To do this, consider the Frobenius morphism $\sigma$ of $X$ restricted to $Y$, and note that

$$
Y_{\sigma^{e}}=C_{X_{\sigma^{e}}}(\gamma)= \begin{cases}\operatorname{PGSp}_{n}(q) & \text { if } n \text { is even } \\ \operatorname{PSO}_{n}(q)=\mathrm{PO}_{n}(q) & \text { otherwise. }\end{cases}
$$

Now $\iota \sigma$ is $\operatorname{PGL}_{n}(q)$-conjugate to $\gamma \sigma$, so let us assume that $g=\gamma \sigma$. Note that $\gamma \sigma=\sigma \gamma$.

By the theory of Shintani descent (see Lemma 2.13), there is a bijective map $f$ between the set of $Y_{\sigma^{e}}$-classes in the coset $\sigma Y_{\sigma^{e}}$ and the set of $Y_{\sigma^{-}}$ classes in $Y_{\sigma}$. Moreover, by arguing as in the proof of Lemma 4.2, we deduce that if $y \in Y_{\sigma}$ has determinant 1 , then there exists $s \in Y_{\sigma^{e}} \operatorname{such}$ that $\operatorname{det}(s)=$ 1 and $f(\sigma s)=y$. (That is, $f$ maps the $Y_{\sigma^{e}}$-class of $\sigma s$ to the $Y_{\sigma}$-class of $y$.) Now $\sigma s$ commutes with $\gamma$, so $(\gamma \sigma s)^{2 e}$ is conjugate to $y^{2}$. Therefore, if $y \in \operatorname{PSp}_{n}\left(q_{0}\right)$ or $\mathrm{PSO}_{n}\left(q_{0}\right)$, in the cases $n$ even and odd, respectively, then there exists $s \in G_{0}$ such that $(g s)^{2 e}$ is conjugate to $y^{2}$. Moreover, since $(\gamma \sigma s)^{2}=\sigma^{2} s^{\sigma} s$ and $\sigma^{2}$ acts as a field automorphism of order $e$ on $\operatorname{PGL}_{n}(q)$ (since $e$ is odd), we can use Corollary 2.15 and Proposition 2.16 to control the maximal subgroups of $G$ containing $y^{2}$.

LEMMA 5.7. With the notation above, suppose that $y \in \operatorname{PSp}_{n}\left(q_{0}\right)$ or $\mathrm{PSO}_{n}\left(q_{0}\right)$ in the respective cases $n$ even and $n$ odd, and fix an element $s \in G_{0}$ such that $(g s)^{2 e}$ is $Y$-conjugate to $y^{2}$. Let $\mathcal{M}$ be the set of maximal subgroups of $G$ containing gs, and assume that $e \geq 3$ is odd. Then the following hold.

(i) There are at most $\left|C_{\mathrm{PGL}_{n}\left(q_{0}\right)}\left(y^{2}\right)\right|$ distinct subgroups in $\mathcal{M}$ of a given type.

(ii) More precisely, the number of $\mathcal{C}_{1}$-subgroups in $\mathcal{M}$ of a given type $\left(P_{n / 2}\right.$, $P_{i, n-i}$, or $\left.\mathrm{GL}_{i}(q) \times \mathrm{GL}_{n-i}(q)\right)$ is at most the number of reducible subgroups of the same type in $\mathrm{PGL}_{n}\left(q_{0}\right)$ containing $y^{2}$.

Proof. First, consider (i). Set $G_{1}=\left\langle\operatorname{PGL}_{n}(q), \gamma \sigma\right\rangle$, and note that all subgroups $H \in \mathcal{M}$ of a given type are $G_{1}$-conjugate. Let $H$ be a maximal subgroup of $G$ containing $\gamma \sigma s$, and let $N$ be the number of subgroups of type $H$ in $\mathcal{M}$. Then $H$ also contains $(\gamma \sigma s)^{2}=\sigma^{2} t$, where $t=s^{\sigma} s$, so

$$
N \leq \frac{\left|\left(\sigma^{2} t\right)^{G_{1}} \cap H\right|}{\left|\left(\sigma^{2} t\right)^{G_{1}}\right|} \cdot \frac{\left|G_{1}\right|}{|H|} .
$$


Further, since $\left|\left(\sigma^{2} t\right)^{G_{1}} \cap H\right| \leq|H| / e$, we have

$$
N \leq\left|C_{G_{1}}\left(\sigma^{2} t\right)\right| / e=\left|C_{G_{1}}\left((\sigma s)^{2}\right)\right| / e .
$$

Next, let $j$ be an integer such that $2 j \equiv 1(\bmod e)$ and $(j,|G|)=1$. (For instance, let $j$ be a solution to the system of congruences $j \equiv a(\bmod e)$ (where $a$ is the multiplicative inverse of 2 in $\mathbb{Z} / e \mathbb{Z})$ and $j \equiv 1\left(\bmod p_{i}\right)$ for all prime divisors $p_{i}$ of $|G|$ with $\left(p_{i}, e\right)=1$; a solution exists by the Chinese remainder theorem.) Now

$$
C_{G_{1}}\left((\sigma s)^{2}\right) \leq C_{G_{1}}\left((\sigma s)^{2 j}\right)
$$

(in fact equality holds), and $(\sigma s)^{2 j} \in \sigma \mathrm{PGL}_{n}(q)$, so Lemma 2.13(i) implies that

$$
\left|C_{G_{1}}\left((\sigma s)^{2 j}\right)\right|=e\left|C_{\mathrm{PGL}_{n}\left(q_{0}\right)}\left(b(\sigma s)^{2 j e} b^{-1}\right)\right|
$$

for some $b \in X$. Since $(j,|G|)=1$, there exists an integer $k$ such that $j k \equiv 1$ $(\bmod |G|)$, and we deduce that

$$
\begin{aligned}
e\left|C_{\mathrm{PGL}_{n}\left(q_{0}\right)}\left(b(\sigma s)^{2 j e} b^{-1}\right)\right| & \leq e\left|C_{\mathrm{PGL}_{n}\left(q_{0}\right)}\left(b(\sigma s)^{2 j k e} b^{-1}\right)\right| \\
& =e\left|C_{\mathrm{PGL}_{n}\left(q_{0}\right)}\left(b(\sigma s)^{2 e} b^{-1}\right)\right| .
\end{aligned}
$$

However, $b(\sigma s)^{2 e} b^{-1}$ and $y^{2}$ are $\operatorname{PGL}_{n}\left(q_{0}\right)$-conjugate, so

$$
e\left|C_{\mathrm{PGL}_{n}\left(q_{0}\right)}\left(b(\sigma s)^{2 e} b^{-1}\right)\right|=e\left|C_{\mathrm{PGL}_{n}\left(q_{0}\right)}\left(y^{2}\right)\right|,
$$

and the result follows.

Finally, let us turn to (ii). Define the integers $j$ and $k$ as above. Let $H$ be a maximal $\mathcal{C}_{1}$-subgroup of $G$ containing $\gamma \sigma s$, say, of type $T$. Then $(\gamma \sigma s)^{2}=(\sigma s)^{2} \in H$; hence, $(\sigma s)^{2 j} \in H$. Since $(\sigma s)^{2 j} \in \sigma \operatorname{PGL}_{n}(q)$, Corollary 2.15 implies that the number of subgroups of $G$ of type $T$ containing $(\sigma s)^{2 j}$ is the same as the number of subgroups of type $T$ in $\operatorname{PGL}_{n}\left(q_{0}\right)$ containing $b(\sigma s)^{2 j e} b^{-1}$. But any such subgroup containing $b(\sigma s)^{2 j e} b^{-1}$ also contains $b(\sigma s)^{2 j k e} b^{-1}$, and this element is $\operatorname{PGL}_{n}\left(q_{0}\right)$-conjugate to $y^{2}$. This completes the proof of the lemma.

We now partition the proof of Theorem 5.1 (with $e$ odd) into several subcases. To do this, let us first define two sets of special cases $\left(n, q_{0}\right)$, which we will consider separately: 


$$
\begin{aligned}
& \mathcal{A}=\{(5,2),(5,3),(7,2),(7,3),(9,2),(11,2),(13,2),(15,2)\} \\
& \mathcal{B}=\{(4,2),(4,3),(6,2),(6,3),(8,2),(10,2),(12,2),(14,2)\}
\end{aligned}
$$

We start by assuming that $n \geq 5$ is odd.

Proposition 5.8. Theorem 5.1 holds when $e$ is odd, $n \geq 5$ is odd, and $\left(n, q_{0}\right) \notin \mathcal{A}$.

Proof. Let $n=2^{k_{1}}+2^{k_{2}}+\cdots+1$ be the binary representation of $n$ (where $k_{i}>k_{i+1}$ for all $\left.i\right)$, and note that $2^{k_{1}}>n / 2$. Set $y=\left[A_{2^{k_{1}}}, A_{2^{k_{2}}}, \ldots, A_{1}\right] \in$ $\mathrm{PSO}_{n}\left(q_{0}\right)$, where $A_{1} \in \mathbb{F}_{q_{0}}^{*}$ and each $A_{m} \in O_{m}^{-}\left(q_{0}\right)$ (with $m>1$ ) is irreducible of order $q_{0}^{m / 2}+1$. Since $\operatorname{det}(y)=1$, there exists $s \in G_{0}$ such that $(g s)^{2 e}$ and $y^{2}$ are conjugate. As usual, let $\mathcal{M}$ be the set of maximal subgroups of $G$ containing $g s$.

The order of some power of $y$ is a primitive prime divisor of $q_{0}^{2^{k_{1}}}-1$. Moreover, since $e$ is odd and $\left(n, q_{0}\right) \notin \mathcal{A},[24$, Lemma 2.1] implies that some power of $y$ has order $r$, where either $r$ is a primitive prime divisor of $q^{2^{k_{1}}}-1$ with $r>2^{k_{1}+1}+1$, or $r$ is a product of primitive prime divisors of $q^{2^{k_{1}}}-1$. Therefore, we can use Theorem 2.12 to restrict the possible subgroups in $\mathcal{M}$. Furthermore, each $H \in \mathcal{M}$ contains a conjugate of $y^{2}$, and so by studying the maximal subgroups of $G$ containing $y^{2}$, we can further restrict the possibilities in $\mathcal{M}$.

By Lemma 5.7(ii), the maximal $\mathcal{C}_{1}$-subgroups containing $y^{2}$ are as follows: one each of type $P_{1, n-1}$ and $\mathrm{GL}_{1}(q) \times \mathrm{GL}_{n-1}(q)$, together with at most one of type $P_{j, n-j}$ and also at most one of type $\mathrm{GL}_{j}(q) \times \mathrm{GL}_{n-j}(q)$ for all $2 \leq j<n / 2$. In particular, by applying Theorem 2.7, we deduce that the entire contribution to $\alpha(z)$ from reducible subgroups is less than

$$
2 q^{-1}+2 q^{1-n}+\sum_{j \geq 2} 4 q^{-j}=2 q^{-1}+2 q^{1-n}+4 /\left(q^{2}-q\right) .
$$

Now assume that $H \in \mathcal{M}$ is irreducible. In view of Theorem 2.12, it follows that $H$ is a $\mathcal{C}_{3^{-}}, \mathcal{C}_{5^{-}}$, or $\mathcal{C}_{8^{-}}$subgroup of $G$. We can immediately eliminate $\mathcal{C}_{3}$-subgroups since $y^{2}$ has only one eigenvalue in $\mathbb{F}_{q_{0}}$. Similarly, there are no $\mathcal{C}_{8}$-subgroups of type $\mathrm{GU}_{n}\left(q^{1 / 2}\right)$ since $y^{2}$ acts irreducibly on a $2^{k_{1}}$-dimensional subspace of $V$. By applying Lemma 5.7, we see that $\mathcal{M}$ contains at most $\left|C_{\mathrm{PGL}_{n}\left(q_{0}\right)}\left(y^{2}\right)\right|<q_{0}^{n-1}$ subfield subgroups for each prime divisor of $e$, and at most the same number of $\mathcal{C}_{8}$-subgroups of type $O_{n}(q)$ (when $q$ is odd). 
Therefore, if $z \in G$ has prime order, then

$$
\alpha(z)<2 q^{-1}+2 q^{1-n}+4 /\left(q^{2}-q\right)+q^{(n-1) / e}((2, q-1)+\log (e)) \cdot f(n, q)
$$

which is less than $1 / 2$. (Note that $(n, q) \neq(5,8)$ since we are assuming that $\left(n, q_{0}\right) \notin \mathcal{A}$. $)$

From the previous bound, it is clear that $\alpha(z) \rightarrow 0$ as $q \rightarrow \infty$, so the asymptotic statement in Theorem 5.1 also holds. However, we claim that if $q$ is bounded, then $\alpha(z)$ does not tend to zero as $n \rightarrow \infty$. To do this, we will prove that if $n e$ is odd, then every $g s \in g \mathrm{PGL}_{n}(q)$ stabilizes a pair of subspaces $(U, W)$ of $V$, where $\operatorname{dim} U=1$ and $\operatorname{dim} W=n-1$. In particular, every such element is contained in a reducible subgroup of type $P_{1, n-1}$ or $\mathrm{GL}_{1}(q) \times \mathrm{GL}_{n-1}(q)$, so $\alpha(z)>q^{-3}$ if $z \in G_{0}$ is a transvection.

Without loss of generality, we may assume that $g=\iota \sigma$. For any $s \in$ $\mathrm{PGL}_{n}(q)$, we have

$$
(g s)^{2 e}=s^{g^{2 e-1}} s^{g^{2 e-2}} \cdots s^{g} s,
$$

and since $e$ is odd, this is equal to

$$
s^{\sigma^{e-1} \iota} s^{\sigma^{e-2}} \cdots s^{\sigma \iota} s=z^{\iota} z
$$

where $z=s^{\sigma^{e-1}} s^{\sigma^{e-2} \iota} \cdots s^{\sigma \iota} s \in \mathrm{PGL}_{n}(q)$. In particular, $z^{\iota} z$ is $\mathrm{PGL}_{n}(q)$ conjugate to $z^{-\iota} z^{\iota} z z^{\iota}=z z^{\iota}=\left(z^{\iota} z\right)^{\iota}$. But every element in $\operatorname{PGL}_{n}(q)$ is conjugate to its transpose; hence, $z^{\iota} z$ is conjugate to its inverse. Consequently, if $\lambda \in \overline{\mathbb{F}}_{q}$ is an eigenvalue of $z^{\iota} z$ on $V$, then $\lambda^{-1}$ is also an eigenvalue. Since $n$ is odd, it follows that \pm 1 occurs as an eigenvalue of $z^{\iota} z=(g s)^{2 e}$. By the Shintani descent argument used in Lemma 5.7(ii), we deduce that $(g s)^{2}$ stabilizes a 1-dimensional subspace $U$ and is therefore contained in a $P_{1}$ parabolic subgroup $H$ of $G$. It follows that $g s$ normalizes $H \cap H^{g s}$, and we note that $H^{g s}$ stabilizes an $(n-1)$-dimensional subspace $W$. (The inversetranspose automorphism interchanges the stabilizers of $i$-dimensional and $(n-i)$-dimensional subspaces of $V$.) There are two possibilities: if $U \subseteq W$, then $H \cap H^{g s}$ is a type $P_{1, n-1}$ maximal parabolic subgroup of $G$ containing gs; otherwise, $V=U \oplus W$, and $H \cap H^{g s}$ is a maximal subgroup of type $\mathrm{GL}_{1}(q) \times \mathrm{GL}_{n-1}(q)$ containing $g s$. This justifies the claim; namely, if $q$ is bounded, then $\alpha(z)$ does not tend to zero as $n$ tends to infinity.

In fact, we claim that if $n \geq 5$, then $s(G)<(q+1)^{2}$, so $u(G)$ is bounded if $q$ is bounded. (We thank Bob Guralnick for suggesting the following argument.) First, fix a basis $\left\{v_{1}, \ldots, v_{n}\right\}$ for $V$, and write $\mathbb{F}_{q}^{*}=\langle\omega\rangle$. Define $q+1$ 
hyperplanes $H_{0}, \ldots, H_{q}$ as follows:

$$
\begin{aligned}
& H_{0}=\left\langle v_{1}, v_{3}, \ldots, v_{n}\right\rangle, \\
& H_{i}=\left\langle v_{1}+\omega^{i} v_{2}, v_{3}, \ldots, v_{n}\right\rangle \quad(1 \leq i \leq q-1), \quad H_{q}=\left\langle v_{2}, \ldots, v_{n}\right\rangle .
\end{aligned}
$$

Consider $\bigcap_{i} H_{i}=\left\langle v_{3}, \ldots, v_{n}\right\rangle$, and note that $\bigcup_{i} H_{i}=V$. Set $W=\left\langle v_{3}, v_{4}\right\rangle$, and label the 1-dimensional subspaces of $W$ as follows:

$$
L_{0}=\left\langle v_{3}\right\rangle, \quad L_{i}=\left\langle v_{3}+\omega^{i} v_{4}\right\rangle \quad(1 \leq i \leq q-1), \quad L_{q}=\left\langle v_{4}\right\rangle
$$

For each $H_{i}$ and $L_{j}$, let $z_{i j}$ be the transvection in $G_{0}$ with center $H_{i}$ and axis $L_{j}$, so $L_{j}=\left[V, z_{i j}\right] \subset C_{V}\left(z_{i j}\right)=H_{i}$. Set $\mathcal{Z}=\left\{z_{i j} \mid 0 \leq i, j \leq q\right\}$.

Let $H$ be a hyperplane in $V$, and let $L$ be a 1-dimensional subspace of $V$. Note that $\operatorname{dim}(H \cap W) \geq 1$ and that $L \subset H_{i}$ for some $i$ (since $V=\bigcup_{i} H_{i}$ ). In particular, some $L_{j}$ is contained in $H \cap W$. Consider the transvection $z_{i j}$. By definition, $z_{i j}$ acts trivially on $H_{i}$, so $z_{i j}$ fixes $L$. In addition, since $\left[V, z_{i j}\right]=L_{j}$, it follows that $z_{i j}$ also fixes $H$, whence $z_{i j}$ fixes the pair of subspaces $(L, H)$.

Now, if $s(G) \geq(q+1)^{2}$, then there exists an element $y \in G$ such that $G=\langle z, y\rangle$ for all $z \in \mathcal{Z}$. Necessarily, $y=g s$ for some $s \in G_{0}$, and recall that we have previously observed that every such element fixes a pair of subspaces $(L, H)$, where $\operatorname{dim} L=1$ and $\operatorname{dim} H=n-1$. By the previous argument, there exists $z \in \mathcal{Z}$ also fixing the pair $(L, H)$, so $\langle z, y\rangle$ is contained in the $G$-stabilizer of $(L, H)$, and thus $G \neq\langle z, y\rangle$. This is a contradiction; hence, $s(G)<(q+1)^{2}$ as claimed.

Proposition 5.9. Theorem 5.1 holds when $e$ is odd, $n$ is even, and $\left(n, q_{0}\right) \notin \mathcal{B}$.

Proof. This is similar to the proof of Proposition 5.8. Let $n=2^{k_{1}}+\cdots+$ $2^{k_{\ell}}$ be the binary representation of $n$ (with $k_{i}>k_{i+1}$ for all $i$ ), and set $y=\left[A_{2^{k_{1}}}, A_{2^{k_{2}}}, \ldots, A_{2^{k_{\ell}}}\right] \in \operatorname{PSp}_{n}\left(q_{0}\right)$, where each $A_{m} \in \operatorname{GSp}_{m}\left(q_{0}\right)$ is irreducible of order $q_{0}^{m / 2}+1$. As in the proof of Proposition 5.8, Theorem 2.12 implies that each irreducible $H \in \mathcal{M}$ is a $\mathcal{C}_{3^{-}}, \mathcal{C}_{5^{-}}$, or $\mathcal{C}_{8^{-}}$subgroup of $G$. More precisely, $H$ is one of the following types:

$$
\mathrm{GL}_{n / 2}\left(q^{2}\right), \mathrm{GL}_{n}\left(q^{1 / r}\right), \operatorname{Sp}_{n}(q), O_{n}^{\epsilon}(q)
$$

(in the latter case $q$ is odd $\epsilon= \pm$, but not both) and Lemma 5.7 implies that there are at most $\left|C_{\mathrm{PGL}_{n}\left(q_{0}\right)}\left(y^{2}\right)\right|<2 q_{0}^{n-1}$ subgroups of each type in $\mathcal{M}$. The 
reducible subgroups in $\mathcal{M}$ can be determined via Lemma 5.7(ii): there is at most one of type $P_{j, n-j}$ and one of type $\mathrm{GL}_{j}(q) \times \mathrm{GL}_{n-j}(q)$, for each even integer $j \leq n / 2$. In particular, if $z \in G$ has prime order, then Theorem 2.7 implies that the contribution to $\alpha(z)$ from reducible subgroups is less than $\sum_{t \geq 1} 4 q^{-2 t}=4 /\left(q^{2}-1\right)$. Consequently, if $n \geq 6$, then Corollary 2.9 implies that

$$
\alpha(z)<4 /\left(q^{2}-1\right)+2 q^{(n-1) / e}(3+\log (e)) \cdot f(n, q)<1 / 2 .
$$

Finally, let us assume that $n=4$. Since $\left(n, q_{0}\right) \notin \mathcal{B}$, we may assume that $q_{0} \geq 4$. Here $\left|C_{\mathrm{PGL}_{4}\left(q_{0}\right)}(y)\right|=\left(q_{0}^{4}-1\right) /\left(q_{0}-1\right)$, and $y^{2}$ is irreducible, so each $H \in \mathcal{M}$ is irreducible, and the possible types are given in (23). In addition, we note that if $H \in \mathcal{M}$ is a $\mathcal{C}_{3}$-subgroup of type $\mathrm{GL}_{2}\left(q^{2}\right)$, then $C_{G}\left(y^{2}\right)=C_{H}\left(y^{2}\right)$ and $\left(y^{2}\right)^{G} \cap H=\left(y^{2}\right)^{H}$, so there is a unique such subgroup in $\mathcal{M}$. By applying the relevant fixed point ratio estimates in Lemma 2.11, we deduce that

$$
\begin{aligned}
\alpha(z)< & \frac{d_{1}\left(q^{3}+2 q+1\right)}{q^{2}\left(q^{3}-1\right)} \\
& +\left(\frac{q_{0}^{4}-1}{q_{0}-1}\right)\left(\frac{q^{2}}{d_{2}\left(q^{3}-1\right)}+\frac{4 d_{2}\left(d_{2}-1\right)}{q^{3}-1}+\log (e) \cdot f(4, q)\right),
\end{aligned}
$$

where $d_{1}=(4, q-1)$ and $d_{2}=(2, q-1)$. It follows that $\alpha(z)<1 / 2$ for all $e \geq 5$.

To deal with the case $(n, e)=(4,3)$, we need to improve the upper bound on the number of subgroups of type $\operatorname{Sp}_{4}(q)$ in $\mathcal{M}$. We claim that for any $e$, there are at most $d_{2}^{2}\left(q_{0}+1\right)$ such subgroups.

To see this, let $G_{1}=\left\langle\mathrm{PGL}_{4}(q), g s\right\rangle$, and observe that all subgroups of $G_{1}$ of type $\operatorname{Sp}_{4}(q)$ are $G_{1}$-conjugate. We may assume that $H \cap \operatorname{PGL}_{4}(q)$ is contained in $C_{\mathrm{PGL}_{4}(q)}(\gamma)=Y_{\sigma^{e}}$. As in the proof of Lemma 5.7, let $j$ be an integer such that $2 j \equiv 1(\bmod e)$ and $\left(j,\left|G_{1}\right|\right)=1$. Write $(g s)^{2}=\sigma^{2} t$, where $t=s^{\sigma} s$, and set $x_{1}:=(g s)^{2 j}=\left(\sigma^{2} t\right)^{j} \in \sigma Y_{\sigma^{e}}$. Note that $x_{1}$ has the same order as $\sigma^{2} t$ since $\left(j,\left|G_{1}\right|\right)=1$. We will count the number of subgroups of type $\operatorname{Sp}_{4}(q)$ containing $x_{1}$. Suppose that $x_{2} \in x_{1}^{G_{1}} \cap H$. Now $x_{1}, x_{2} \in \sigma Y_{\sigma^{e}}$, so we can consider their images $f\left(x_{1}\right), f\left(x_{2}\right)$ in $Y_{\sigma}$ under the corresponding Shintani map $f$. Since the $x_{i}$ are $G_{1}$-conjugate, it follows that the $f\left(x_{i}\right)$ are irreducible and have the same eigenvalues. Therefore, $f\left(x_{1}\right)$ and $f\left(x_{2}\right)$ are

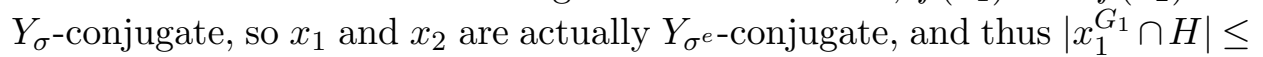
$\left|x_{1}^{Y_{\sigma}{ }^{e}}\right|$. It follows that the number of subgroups of type $\operatorname{Sp}_{4}(q)$ in $\mathcal{M}$ is at 
most

$$
\frac{\left|x_{1}^{G_{1}} \cap H\right|}{\left|x_{1}^{G_{1}}\right|} \cdot \frac{\left|G_{1}\right|}{|H|} \leq \frac{\left|x_{1}^{Y_{\sigma^{e}}}\right|}{\left|x_{1}^{G_{1}}\right|} \cdot \frac{\left|G_{1}\right|}{|H|}=\frac{\left|Y_{\sigma^{e}}\right|}{|H|} \cdot \frac{\left|C_{G_{1}}\left(x_{1}\right)\right|}{\left|C_{Y_{\sigma^{e}}}\left(x_{1}\right)\right|} .
$$

Further, by considering the Shintani map between $\mathrm{PGL}_{4}(q)$-classes in $\sigma \mathrm{PGL}_{4}(q)$ and $\mathrm{PGL}_{4}\left(q_{0}\right)$-classes in $\mathrm{PGL}_{4}\left(q_{0}\right)$, we deduce that

$$
\left|C_{G_{1}}\left(x_{1}\right)\right|=2 e\left|C_{\mathrm{PGL}_{4}(q)}\left(x_{1}\right)\right|=2 e\left|C_{\mathrm{PGL}_{4}\left(q_{0}\right)}(y)\right|=2 e\left(\frac{q_{0}^{4}-1}{q_{0}-1}\right)
$$

(see Lemma 2.13(i)). We also note that $x_{1}^{e} \in C_{Y_{\sigma} e}\left(x_{1}\right)$ and that $\left|x_{1}^{e}\right|=|y|=$ $\left(q_{0}^{2}+1\right) / d$, so $\left|C_{Y_{\sigma e}}\left(x_{1}\right)\right| \geq\left(q_{0}^{2}+1\right) / d_{2}$. Finally, since $|H| \geq 2 e\left|\operatorname{PSp}_{4}(q)\right|=$ $2 e\left|Y_{\sigma^{e}}\right| / d_{2}$, we conclude that

$$
\frac{\left|Y_{\sigma^{e}}\right|}{|H|} \cdot \frac{\left|C_{G_{1}}\left(x_{1}\right)\right|}{\left|C_{Y_{\sigma^{e}}}\left(x_{1}\right)\right|} \leq d_{2}^{2}\left(q_{0}+1\right) .
$$

This justifies the claim. In particular, for $(n, e)=(4,3)$, we have

$$
\begin{aligned}
\alpha(z)< & \frac{d_{1}\left(q^{3}+2 q+1\right)}{q^{2}\left(q^{3}-1\right)}+\left(\frac{q_{0}^{4}-1}{q_{0}-1}\right)\left(\frac{4 d_{2}\left(d_{2}-1\right)}{q^{3}-1}+f(4, q)\right) \\
& +d_{2}\left(q_{0}+1\right) \cdot \frac{q^{2}}{q^{3}-1},
\end{aligned}
$$

and the result follows.

It is easy to check that the above bounds on $\alpha(z)$ imply that $\alpha(z) \rightarrow 0$ (and thus $u(G) \rightarrow \infty$ ) as $q \rightarrow \infty$. In contrast to the situation in Proposition 5.8, we claim that $u(G)$ also tends to infinity if $q$ is bounded and $n \rightarrow \infty$.

To see this, we may assume that $q$ (and therefore $e$ ) is bounded and that $n$ is large compared with $e$. Let $k$ be an even integer such that $n / 4<k<n / 2$, $(k, e)=(n-k, e)=1$, and $(k, n-k)=2$. Set $y=[A, B] \in \operatorname{PSp}_{n}\left(q_{0}\right)$, where $A$ and $B$ are irreducible of dimensions $n-k$ and $k$, respectively. Since $(k, e)=$ $(n-k, e)=1$, it follows that $A$ and $B$ remain irreducible over $\mathbb{F}_{q}$. In addition, some power of $y$ has order $r$, where either $r>2(n-k)+1$ is a primitive prime divisor of $q^{n-k}-1$, or $r$ is a product of primitive prime divisors of $q^{n-k}-1$. If $H \in \mathcal{M}$ is reducible, then $H$ is of type $\operatorname{GL}_{k}(q) \times \mathrm{GL}_{n-k}(q)$, and there is a unique such subgroup in $\mathcal{M}$. By Theorem 2.12, the irreducible subgroups in $\mathcal{M}$ are of type $\mathrm{GL}_{n / 2}\left(q^{2}\right), \mathrm{GL}_{n}\left(q_{1}\right), \operatorname{Sp}_{n}(q)$, or $O_{n}^{+}(q)$, where $q=q_{1}^{a}$ for some 
prime divisor $a$ of $e$. Since $\left|C_{\mathrm{PGL}_{n}\left(q_{0}\right)}\left(y^{2}\right)\right| \leq 2\left(q_{0}^{n-k}-1\right)\left(q_{0}^{k}-1\right) /\left(q_{0}-1\right)$, we conclude that

$$
\alpha(z)<2 q^{-k}+\frac{2(3+\log (e))\left(q_{0}^{n-k}-1\right)\left(q_{0}^{k}-1\right)}{q_{0}-1} \cdot f(n, q),
$$

which tends to 0 as $n \rightarrow \infty$.

To complete the proof of Theorem 5.1, it remains to deal with the cases $\left(n, q_{0}\right)$ in $\mathcal{A}$ and $\mathcal{B}$, together with the case $n=3$.

Proposition 5.10. Theorem 5.1 holds when e is odd and $\left(n, q_{0}\right) \in \mathcal{A} \cup \mathcal{B}$.

Proof. Define the element $y$ as in the proof of Propositions 5.8 and 5.9, according to the parity of $n$. As before, note that some power of $y$ has order $r$, where $r$ is a primitive prime divisor of $q^{2^{k_{1}}}-1$, so we can use the main theorem of [25] to restrict the subgroups containing gs. Also note that $\left(2^{k_{1}}, r\right)=(4,5)$ if $n \leq 7$; otherwise, $\left(2^{k_{1}}, r\right)=(8,17)$. As usual, let $\mathcal{M}$ denote the set of maximal subgroups of $G$ containing $g s$. We now inspect the various subgroup collections presented in [25, Section 2].

First, assume that $n$ is odd. As in the proof of Proposition 5.8, the contribution to $\alpha(z)$ from reducible subgroups is less than $2 q^{-1}+2 q^{1-n}+4 /\left(q^{2}-\right.$ q). If $H \in \mathcal{M}$ is a $\mathcal{C}_{2}$-subgroup, then [25, Example 2.3] implies that $n \leq 7$ and that $H$ is of type $\mathrm{GL}_{1}(q)<S_{n}$. By considering the eigenvalues of $y$, we can eliminate $\mathcal{C}_{3^{-}}$-subgroups, while $\mathcal{C}_{4^{-}}, \mathcal{C}_{6^{-}}$, and $\mathcal{C}_{7}$-subgroups are ruled out by [25]. As usual, $\mathcal{M}$ contains subfield subgroups of type $\operatorname{GL}_{n}\left(q_{1}\right)$, where $q=q_{1}^{a}$ and $a$ is a prime divisor of $e$; for each divisor $a$, there are at most $\left|C_{\mathrm{PGL}_{n}\left(q_{0}\right)}\left(y^{2}\right)\right|<q_{0}^{n-1}$ such subgroups in $\mathcal{M}$ (see Lemma 5.7(i)). Similarly, the only $\mathcal{C}_{8}$-subgroups in $\mathcal{M}$ are of type $O_{n}(q)$ (assuming that $q$ is odd), and again, there are fewer than $q_{0}^{n-1}$ such subgroups.

To complete the analysis of $\mathcal{M}$ when $n$ is odd, we may assume that $H \in \mathcal{M}$ is a $\mathcal{C}_{9}$-subgroup. The various possibilities are listed in [25, Tables $2-8]$, and we inspect each table in turn. Let $H_{0}$ denote the socle of $H$. Since $q \neq p$, by [5, Table 7.19] we may assume that $n \geq 7$.

By inspecting [25, Tables 2-5], we can quickly rule out any possibilities with $H_{0}$ an alternating or sporadic group. (Here it is helpful to note that if $q_{0}$ is prime (in terms of the notation in [25] - this number is listed in the sixth column of the relevant tables), then the corresponding almost simple subgroup $H$ is contained in a proper subfield subgroup of $G$; hence, $H$ is nonmaximal.) Similarly, we can rule out the cases appearing in [25, Tables 
6 and 7]. It remains to deal with the cases listed in [25, Table 8]; here $H_{0}$ belongs to an infinite family of simple classical groups in characteristic $p^{\prime} \neq p$. Since $n \geq 7$ is odd and $2^{k_{1}}=4$ or 8 , we can quickly eliminate all cases unless $\left(n, q_{0}\right)=(9,2)$ and $H_{0}=\mathrm{PSL}_{2}(17)$. However, the 2 -modular character table of $\mathrm{SL}_{2}(17)$ (see [29, p. 11]) indicates that $\mathrm{SL}_{2}(17)$ does not admit a 9dimensional irreducible representation in characteristic 2 , so this possibility is also eliminated. We conclude that there are no $\mathcal{C}_{9}$-subgroups in $\mathcal{M}$.

It follows that if $n \geq 5$ is odd and $z \in G$ is an element of prime order, then (22) holds, and thus we reduce to the case $(n, q)=(5,8)$. However, if $n=5$, then the only reducible subgroups in $\mathcal{M}$ are those of type $P_{1,4}$ and $\mathrm{GL}_{1}(q) \times \mathrm{GL}_{4}(q)$, so we can omit the $4 /\left(q^{2}-q\right)$ term in the upper bound in $(22)$, and this yields $\alpha(z)<1 / 2$.

Now assume that $n$ is even. As above, by inspecting [25] we deduce that if $H \in \mathcal{M}$, then either $H$ is a subfield subgroup, a $\mathcal{C}_{3}$-subgroup of type $\mathrm{GL}_{n / 2}\left(q^{2}\right)$, a $\mathcal{C}_{8}$-subgroup of type $O_{n}^{\epsilon}(q)$ ( $q$ odd), or $\operatorname{Sp}_{n}(q)$, or $n=6$ and $H$ is a $\mathcal{C}_{2}$-subgroup of type $\mathrm{GL}_{1}(q) \imath S_{6}$. Therefore, if $n \geq 6$, we have

$$
\alpha(z)<4 /\left(q^{2}-1\right)+2 q^{(n-1) / e}((2, q-1)+3+\log (e)) \cdot f(n, q)<1 / 2,
$$

and it is easy to see that $\alpha(z) \rightarrow 0$ as $q \rightarrow \infty$.

Finally, let us assume that $n=4$. Suppose that $q_{0}=2$, and note that we may assume that $q \geq 32$ (see Proposition 2.17). Now $\left|C_{\mathrm{PGL}_{4}\left(q_{0}\right)}\left(y^{2}\right)\right|=15$, and by applying the bounds in Lemma 2.11, using the fact that $y^{2}$ is irreducible and belongs to a unique $\mathcal{C}_{3}$-subgroup of type $\operatorname{GL}_{2}\left(q^{2}\right)$, we deduce that

$$
\alpha(z)<\frac{q^{3}+2 q+1}{q^{2}\left(q^{3}-1\right)}+15\left(\frac{q^{2}}{q^{3}-1}+\log (e) \cdot f(4, q)\right)<1 / 2
$$

if $q>32$. Finally, if $(n, q)=(4,32)$, then we can replace the term $\log (e) f(4, q)$ in the above bound by $1 / 1198336$. (Indeed, if $H$ is a subfield subgroup of type $\mathrm{GL}_{4}(2)$, then it is easy to check that $\operatorname{fpr}(z, G / H) \leq 1 / 1198336$ for all $z \in G$ of prime order.) This yields $\alpha(z)<1 / 2$. Similarly, if $q_{0}=3$, then $\left|C_{\mathrm{PGL}_{4}\left(q_{0}\right)}\left(y^{2}\right)\right|=40$, and we have

$$
\alpha(z)<\frac{(4, q-1) \cdot\left(q^{3}+2 q+1\right)}{q^{2}\left(q^{3}-1\right)}+40\left(\frac{q^{2}}{2\left(q^{3}-1\right)}+\frac{8}{q^{3}-1}+\log (e) \cdot f(4, q)\right),
$$

which is less than $1 / 2$ unless $(n, q)=(4,27)$. As in the proof of Proposition 5.9 , if $(n, q)=(4,27)$, then there are at most $2^{2}(3+1)=16$ subgroups 
of type $\mathrm{Sp}_{4}(q)$ in $\mathcal{M}$, whence

$$
\alpha(z)<\frac{19738}{7174089}+\frac{2916}{9841}+\frac{160}{9841}+40 \cdot f(4,27)<1 / 2
$$

as required.

Proposition 5.11. Theorem 5.1 holds when $e$ is odd and $n=3$.

Proof. In view of Proposition 2.17, we may assume that $q \geq 27$. Set $y=$ $\left[A_{2}, A_{1}\right] \in \mathrm{PO}_{3}\left(q_{0}\right)$, where $A_{2} \in O_{2}^{-}\left(q_{0}\right)$ is irreducible (over both $\mathbb{F}_{q_{0}}$ and $\mathbb{F}_{q}$ ). By inspecting the explicit list of maximal subgroups of $G$ given in [5, Table 7.3], we deduce that the possibilities for $H \in \mathcal{M}$ are as follows: we get reducible subgroups of type $P_{1,2}$ and $\mathrm{GL}_{1}(q) \times \mathrm{GL}_{2}(q)$ (exactly one of each type), $\mathcal{C}_{2}$-subgroups of type $\mathrm{GL}_{1}(q) 2 S_{3}$, subfield subgroups of type $\mathrm{GL}_{3}\left(q_{1}\right)$, and $\mathcal{C}_{8}$-subgroups of type $O_{3}(q)$ (with $q$ odd). Now $\left|C_{\mathrm{PGL}_{3}\left(q_{0}\right)}\left(y^{2}\right)\right| \leq q_{0}^{2}-1$, and thus Lemma 2.10 implies that

$$
\alpha(z)<2 q^{-1}+2 q^{-2}+\left(q_{0}^{2}-1\right)((2, q-1)+\log (e))\left(q^{2}+q+1\right)^{-1}<1 / 2
$$

for all $q \geq 27$.

This completes the proof of Theorem 5.1.

\section{§6. Graph automorphisms}

In this section, we complete the proof of Theorems 2-4 by considering the case where $G=\left\langle G_{0}, g\right\rangle$ with $g$ a graph automorphism of $G_{0}$. Here $n \geq 3$, and $g$ is of the form $g=\iota x$, where $\iota$ is the inverse-transpose map and $x \in$ $\operatorname{PGL}_{n}(q)$. As usual, we may replace $x$ by $\delta=\left[\lambda, I_{n-1}\right]$ for some $\lambda \in \mathbb{F}_{q}^{*}$, so $g^{2}=(\iota \delta)^{2}=\delta^{\iota} \delta=1$, and thus $g$ is an involutory graph automorphism.

The $\mathrm{PGL}_{n}(q)$-classes of involutory graph automorphisms of $G_{0}$ are described in Lemma 6.1 below. First, we require some notation. For $n$ even, we define

(24) $S=\left(\begin{array}{ccccc}0 & -1 & & & \\ 1 & 0 & & & \\ & & 0 & -1 & \\ & & 0 & \\ & & & & \ddots\end{array}\right), \quad S^{+}=\left(\begin{array}{ccccc}0 & 1 & & & \\ 1 & 0 & & & \\ & & 0 & 1 & \\ & 1 & 0 & \\ & & & \ddots\end{array}\right)$, 
and $t=\left[J_{2}, I_{n-2}\right]$. In addition, if $n$ is even and $q$ is odd, we set

$$
S^{-}=\left(\begin{array}{ccccccc}
0 & 1 & & & & & \\
1 & 0 & & & & & \\
& & \ddots & & & & \\
& & & 0 & 1 & & \\
& & & 1 & 0 & & \\
& & & & & \mu & \\
& & & & & & 1
\end{array}\right),
$$

where $-\mu / 2 \in \mathbb{F}_{q}$ is a nonsquare.

Lemma 6.1. Let $g \in \operatorname{Aut}\left(G_{0}\right)$ be an involutory graph automorphism.

(i) If $n$ is odd, then $g$ is $\mathrm{PGL}_{n}(q)$-conjugate to $\iota$, and $C_{G_{0}}(\iota)$ is of type $\mathrm{O}_{n}(q)$.

(ii) If $n$ and $q$ are even, then $g$ is $\mathrm{PGL}_{n}(q)$-conjugate to $\iota S$ or $\iota S t$, where $C_{G_{0}}(\iota S)=\operatorname{Sp}_{n}(q)$ and $C_{G_{0}}(\iota S t)=C_{\operatorname{Sp}_{n}(q)}(t)$.

(iii) If $n$ is even and $q$ is odd, then $g$ is $\mathrm{PGL}_{n}(q)$-conjugate to $\iota S, \iota S^{+}$, or $\iota S^{-}$, and the respective centralizers are of type $\operatorname{Sp}_{n}(q), O_{n}^{+}(q)$, and $O_{n}^{-}(q)$.

Proof. This is well known. For example, see [2, Section 19], when $q$ is even, and [34, Lemma 3.7] when $q$ is odd.

Our main result is the following. $\left(\right.$ Here $\operatorname{PSL}_{4}(3) .2_{2} \cong\left\langle\operatorname{PSL}_{4}(3), \iota\right\rangle$, where $\iota$ is the inverse-transpose graph automorphism.)

Theorem 6.2. Let $G_{0}=\operatorname{PSL}_{n}(q)$, and let $G=\left\langle G_{0}, g\right\rangle$, where $g=\iota x$ is the product of the inverse-transpose graph automorphism $\iota$ and $x \in \mathrm{PGL}_{n}(q)$. If we assume that $G \neq \mathrm{PSL}_{4}(2) .2, \mathrm{PSL}_{4}(3) .2_{2}$, then there exists $s \in G_{0}$ such that

$$
\sum_{H \in \mathcal{M}(g s)} \operatorname{fpr}(z, G / H)<1 / 2
$$

for all $z \in G$ of prime order. In particular, $u(G) \geq 2$ for all $G$. Moreover, $u(G)$ is bounded as $|G| \rightarrow \infty$ if and only if $q$ is bounded and $n$ is odd.

REMARK 6.3. The excluded cases $G=\operatorname{PSL}_{4}(2) .2$ and $\operatorname{PSL}_{4}(3) .2_{2}$ are genuine exceptions to the bound in $(26)$, but it is easy to check that $u(G) \geq 2$ (see Proposition 2.18). 
We partition the proof of Theorem 6.2 into a number of subcases. We begin by assuming that $n \geq 5$ is odd.

Proposition 6.4. Theorem 6.2 holds when $n \geq 5$ is odd.

Proof. Let $\mathcal{A}=\{(9,2),(7,2),(5,4),(5,3),(5,2)\}$. If $(n, q) \in \mathcal{A}$, then Proposition 2.17 applies, so we may assume otherwise. Without loss of generality, we may assume that $g=\iota$ (see Lemma 6.1), and thus $C_{G_{0}}(g)$ is of type $O_{n}(q)$. In particular, we may choose $s \in C_{G_{0}}(g)$ such that $s=[A, 1]$ (modulo scalars), where $A \in \mathrm{SO}_{n-1}^{-}(q)$ is irreducible of order $q^{(n-1) / 2}+1$. Set $y=(g s)^{2}=s^{2}=\left[A^{2}, 1\right]$ and note that

$$
|y|=\left(q^{(n-1) / 2}+1\right) /(2, q-1),
$$

so some power of $y$ has order $r$, where $r$ is a primitive prime divisor of $q^{n-1}-1$.

Let $\mathcal{M}$ be the set of maximal subgroups of $G$ containing $g s$, and suppose that $H \in \mathcal{M}$. We claim that one of the following holds:

(i) $H$ is a $\mathcal{C}_{1}$-subgroup of type $\mathrm{GL}_{1}(q) \times \mathrm{GL}_{n-1}(q)$; there is exactly one such subgroup in $\mathcal{M}$;

(ii) $H$ is a $\mathcal{C}_{8}$-subgroup of type $O_{n}(q), q$ is odd, and there are at most $2 q^{(n-1) / 2}$ such subgroups in $\mathcal{M}$.

First, assume that $H$ is reducible. Visibly, $y$ fixes a decomposition $V=$ $U \oplus W$ of the natural $G_{0}$-module $V$, where $\operatorname{dim} U=n-1$. Moreover, $U$ and $W$ are the only proper $y$-invariant subspaces of $V$ since $A^{2}$ acts irreducibly on $U$. Since $H$ is normalized by $g s$, it follows that $\mathcal{M}$ contains a unique reducible subgroup, which is of type $\mathrm{GL}_{1}(q) \times \mathrm{GL}_{n-1}(q)$. For the remainder, we may assume that $H \in \mathcal{M}$ is irreducible; we consider each of the Aschbacher families in turn, using [25] to restrict the possibilities.

If $H$ is a $\mathcal{C}_{2}$-subgroup, then [25, Example 2.3] indicates that $H$ is of type $\mathrm{GL}_{1}(q)$ \ $S_{n}$ with $q \geq 5$, and we quickly deduce that $|y| \leq(q-1) n$. However, this is incompatible with (27) unless $n=5$ and $q \leq 8$. If $n=5$ and $q=5$ or 8 , then $r=13$ does not divide $|H \cap \operatorname{PGL}(V)|$. Similarly, if $(n, q)=(5,7)$, then $|y|=25$, but $|H \cap \operatorname{PGL}(V)|$ is not divisible by 25 , so there are no $\mathcal{C}_{2}$-subgroups in $\mathcal{M}$.

We can eliminate subfield subgroups since $\left|\mathrm{PGL}_{n}\left(q_{1}\right)\right|$ is indivisible by $r$. Similarly, there are no $\mathcal{C}_{8}$-subgroups of type $\mathrm{GU}_{n}\left(q^{1 / 2}\right)$ in $\mathcal{M}$, and the main theorem of [25] immediately rules out any $\mathcal{C}_{4^{-}}, \mathcal{C}_{6^{-}}$, or $\mathcal{C}_{7^{-}}$-subgroups.

By [25, Example 2.4], if $H$ is a $\mathcal{C}_{3}$-subgroup, then $n=r$ and $H$ is of type $\mathrm{GL}_{1}\left(q^{r}\right)$. Here $H \cap \operatorname{PGL}(V) \leq B . r$, where $B$ is a cyclic group of order 
$m=\left(q^{r}-1\right) /(q-1)$. In view of $(27)$, it follows that $|y|>r$ (since $(n, q) \notin$ $\mathcal{A})$, and thus $y^{r} \in B$ is nontrivial. In particular, 1 has multiplicity 1 as an eigenvalue of $y^{r}$, but all the eigenvalues of any element of $B$ have the same multiplicative order in $\mathbb{F}_{q^{r}}^{*}$. This is a contradiction, so there are no $\mathcal{C}_{3}$-subgroups in $\mathcal{M}$.

Next, suppose that $q$ is odd and that $H \in \mathcal{M}$ is a $\mathcal{C}_{8}$-subgroup of type $O_{n}(q)$. Set $c=(n, q-1)$, and let $N$ be the number of distinct $G$-conjugates of $H$ in $\mathcal{M}$, so

$$
N \leq \frac{\left|y^{G} \cap H\right|}{\left|y^{G}\right|} \cdot[G: H]
$$

By [33, Proposition 4.8.4], we have

$$
[G: H]=\frac{\left|\mathrm{SL}_{n}(q)\right|}{c\left|\mathrm{SO}_{n}(q)\right|}<c^{-1} q^{\left(n^{2}+n-2\right) / 2},
$$

and we calculate that

$$
\left|y^{G} \cap H\right|=\frac{\left|\mathrm{SO}_{n}(q)\right|}{q^{(n-1) / 2}+1}<q^{\left(n^{2}-2 n+1\right) / 2}, \quad\left|y^{G}\right|>\frac{1}{2} q^{n^{2}-n} .
$$

Therefore, (28) yields $N<2 c^{-1} q^{(n-1) / 2}$. Finally, since there are at most $c$ distinct $G$-classes of such subgroups (see [33, Proposition 4.8.4]), it follows that there are at most $2 q^{(n-1) / 2}$ subgroups of this type in $\mathcal{M}$.

To complete the analysis of $\mathcal{M}$, we may assume that $H \in \mathcal{C}_{9}$ is almost simple with socle $H_{0}$. According to [25, Example 2.6], $H_{0}$ is not an alternating group. If $H_{0}$ is a sporadic group, then by inspecting [25, Table 5], we reduce to the case $\left(G_{0}, H_{0}\right)=\left(\mathrm{PSL}_{11}(2), \mathrm{M}_{24}\right)$, which we can immediately eliminate since $|y|=33$ by $(27)$ but $|x| \leq 23$ for all $x \in \mathrm{M}_{24}$. Now assume that $H_{0}$ is a simple group of Lie type in characteristic $\ell$. By inspecting [25, Table 6], we see that no cases arise when $\ell=p$, so let us assume that $\ell \neq p$. Here the relevant cases are recorded in [25, Tables 7 and 8], and it is straightforward to rule out them all. For example, suppose that $H_{0}=\operatorname{PSL}_{d}(s)$, where $s$ is an $\ell$-power and $d \geq 3$ is prime. Then $n=r=\left(s^{d}-1\right) /(s-1)$, so $(27)$ implies that

$$
|y|=2^{(n-1) / 2}+1=2^{\left(\left(s^{d}-1\right) /(s-1)-1\right) / 2}+1>s^{d}-1
$$

for all possible $s$ and $d$. However, we have $|x| \leq s^{d}-1$ for all $x \in H \cap \operatorname{PGL}(V)$ (indeed, $|x| \leq s^{d}-1$ for all $x \in \mathrm{GL}_{d}(s)$ - see, e.g., [17, Corollary 2]), so this case does not arise. 
We are now in a position to complete the proof of the proposition. Let $z \in G$ be an element of prime order, and define $\alpha(z)$ as in (14). By applying Proposition 2.8, we deduce that if $(n, q) \notin \mathcal{A}$, then

$$
\alpha(z)<q^{-2}+((2, q-1)-1) \cdot 2 q^{(n-1) / 2} \cdot 2 q^{1-n} \leq q^{-1},
$$

and the result follows.

Note that the above bound implies that $\alpha(z) \rightarrow 0$ as $q \rightarrow \infty$. However, we claim that if $q$ is bounded, then $\alpha(z)$ does not tend to zero as $n$ tends to infinity. To do this, we prove that every element $\iota x \in \iota \mathrm{PGL}_{n}(q)$ is contained in at least one reducible subgroup of type $P_{1, n-1}$ or $\mathrm{GL}_{1}(q) \times \mathrm{GL}_{n-1}(q)$. First, note that if $\lambda \in \overline{\mathbb{F}}_{q}$ is an eigenvalue of $(\iota x)^{2}$ on $V$, then $\lambda^{-1}$ must also occur as an eigenvalue (see [19, Theorem 4.2]). Since $n$ is odd, it follows that $(\iota x)^{2}$ has at least one eigenvalue equal to 1 or -1 . In particular, $(\iota x)^{2}$ stabilizes a 1-dimensional subspace $U$ of $V$, and we complete the argument as in the proof of Proposition 5.8. Moreover, by repeating the final argument in the proof of Proposition 5.8, we deduce that $s(G)<(q+1)^{2}$ if $n \geq 5$, so indeed, we see that $u(G)$ is bounded if $q$ is bounded.

Proposition 6.5. Theorem 6.2 holds when $n \equiv 2(\bmod 4)$.

Proof. Let $\mathcal{B}=\{(10,2),(6,2),(6,3),(6,4)\}$. If $(n, q) \in \mathcal{B}$, then Proposition 2.17 applies, so assume otherwise. Set $t=\left[J_{2}, I_{n-2}\right]$, and define the matrix $S$ as in (24). By Lemma 6.1, if $q$ is even, then there are two $\mathrm{PGL}_{n}(q)$ classes of involutory graph automorphisms, with representatives $\iota S$ and $\iota S t$. Clearly, these representatives are in the same $G_{0}$-coset, so we may assume that $g=\iota S$ and that $C_{G_{0}}(g)$ is of type $\operatorname{Sp}_{n}(q)$. If $q$ is odd and $\operatorname{det}(\delta)=\lambda$ is a square in $\mathbb{F}_{q}$, then we may assume that $g=\iota S$. Similarly, if $\lambda$ is a nonsquare, then we may assume that $g=\iota S \delta$. Now $C_{\mathrm{PGL}_{n}(q)}(\iota S)=\operatorname{PGSp}_{n}(q)$ contains an element $\delta$ of determinant $\lambda^{n / 2}$, so if $\lambda$ is a nonsquare, we may assume that $g=\iota S \delta$ with $\delta \in C_{\mathrm{PGL}_{n}(q)}(\iota S)$.

Set $k=n / 2+1$, so $k$ is even and $(k, n-k)=2$. If $q$ is even, then set $s \in C_{G_{0}}(g)=\operatorname{Sp}_{n}(q)$ with $s=[A, B]$, where $A \in \operatorname{Sp}_{k}(q)$ and $B \in \operatorname{Sp}_{n-k}(q)$ are irreducible. Similarly, if $q$ is odd, we choose $s \in C_{G_{0}}(\iota S)$ so that $s$ (or $\delta s$ if $g=\iota S \delta)$ is of the form $[A, B]$, with $A, B$ as before. For all $q$, we set $y=(g s)^{2}=\left[A^{2}, B^{2}\right]$, and we note that $A^{2}$ and $B^{2}$ are irreducible. Now

$$
|y| \geq\left(q^{k / 2}+1\right) /(2, q-1)^{2},
$$

and we see that some power of $y$ has order $r$, where $r$ is a primitive prime divisor of $p^{f k}-1$ (where $q=p^{f}$ and $p$ is a prime). 
As before, let $\mathcal{M}$ be the set of maximal subgroups of $G$ containing $g s$. We claim that if $H \in \mathcal{M}$, then one of the following holds:

(i) $\quad H$ is a $\mathcal{C}_{1}$-subgroup of type $\mathrm{GL}_{k}(q) \times \mathrm{GL}_{n-k}(q)$; there is exactly one such subgroup in $\mathcal{M}$;

(ii) $H$ is a $\mathcal{C}_{3}$-subgroup of type $\mathrm{GL}_{n / 2}\left(q^{2}\right)$; there is exactly one such subgroup in $\mathcal{M}$;

(iii) $H$ is a $\mathcal{C}_{8}$-subgroup of type $\operatorname{Sp}_{n}(q)$ or $O_{n}^{+}(q)$ ( $q$ odd); there are at most $2 q^{n / 2-1}$ subgroups of each type in $\mathcal{M}$.

To determine the reducible subgroups in $\mathcal{M}$, we argue as in the proof of Proposition 6.4. Now assume that $H$ is irreducible. To determine the possibilities for $H$, we apply the main theorem of [25], considering the various $\mathcal{C}_{i}$ families in turn.

Suppose that $H$ is a $\mathcal{C}_{2}$-subgroup. Then according to [25, Example 2.3], we may assume that $H$ is of type $\mathrm{GL}_{1}(q) \prec S_{n}$, so $H \cap \operatorname{PGL}(V) \leq(q-1)^{n-1} . S_{n}$ and $q \geq 5$ (see [33, Table 3.5.H]). If $(n, q) \neq(10,5)$, then [24, Lemma 2.1] implies that some power of $y$ has order $r^{\prime}$, where either $r^{\prime}$ is a primitive prime divisor of $q^{k}-1$ with $r^{\prime}>2 k+1$, or $r^{\prime}$ is a product of primitive prime divisors of $q^{k}-1$. In this case, $\mathcal{C}_{2}$-subgroups are ruled out by Theorem 2.12. Finally, if $(n, q)=(10,5)$, then the order of $s=[A, B]$ is at least $\operatorname{lcm}\left(5^{3}+1,5^{2}+1\right)=$ 1638 , so $|y| \geq 819$. However, we have $|x| \leq 120$ for all $x \in H \cap \operatorname{PGL}(V)$, so there are no $\mathcal{C}_{2}$-subgroups in $\mathcal{M}$.

Next, suppose that $H \in \mathcal{M}$ is a $\mathcal{C}_{3}$-subgroup. By [25, Example 2.3], $H$ is of type $\mathrm{GL}_{n / 2}\left(q^{2}\right)$ since $(k, n-k)=2$, and [33, Proposition 4.3.6] indicates that $G$ has a unique conjugacy class of such subgroups. Now $y^{G} \cap H=y^{H}$ since any two semisimple elements in $\operatorname{PGL}_{n / 2}\left(q^{2}\right)$ with identical eigenvalues are $\mathrm{PSL}_{n / 2}\left(q^{2}\right)$-conjugate. In addition, $C_{G}(y)$ is of type $Z_{q^{k}-1} \times Z_{q^{n-k}-1}$, so $C_{G}(y)$ is contained in a $\mathcal{C}_{3}$-subgroup of type $\mathrm{GL}_{n / 2}\left(q^{2}\right)$, and therefore we may assume that $C_{G}(y) \leq H$. By applying Corollary 2.5, it follows that $y$ is contained in a unique such subgroup.

By the main theorem of $[25]$, there are no $\mathcal{C}_{4^{-}}, \mathcal{C}_{6^{-}}$, or $\mathcal{C}_{7^{-}}$-subgroups in $\mathcal{M}$, and we can eliminate subfield subgroups and $\mathcal{C}_{8}$-subgroups of type $\mathrm{GU}_{n}\left(q^{1 / 2}\right)$ since $r$ must divide $|H \cap \operatorname{PGL}(V)|$. Next, suppose that $H \in \mathcal{M}$ is a $\mathcal{C}_{8}$-subgroup of type $\operatorname{Sp}_{n}(q)$ or $O_{n}^{\epsilon}(q)$. In the latter case, note that $q$ is odd and that $\epsilon=+$ is the only possibility since $y$ fixes a decomposition $V=U \oplus W$, where $U$ and $W$ are both nondegenerate orthogonal subspaces of minus-type. Suppose that $H \in \mathcal{M}$ is of type $\operatorname{Sp}_{n}(q)$. Set $c=(q-1, n / 2)$, and let $N$ be the number of distinct $G$-conjugates of $H$ containing $y$. By 
[33, Proposition 4.8.3], we have

$$
[G: H]=\frac{\left|\mathrm{SL}_{n}(q)\right|}{c\left|\mathrm{Sp}_{n}(q)\right|}<c^{-1} q^{\left(n^{2}-n-2\right) / 2},
$$

while

$$
\begin{aligned}
\left|y^{G} \cap H\right| & =\frac{\left|\operatorname{Sp}_{n}(q)\right|}{\left(q^{k / 2}+1\right)\left(q^{(n-k) / 2}+1\right)}<q^{n^{2} / 2}, \\
\left|y^{G}\right| & =\frac{\left|\mathrm{GL}_{n}(q)\right|}{\left(q^{k}-1\right)\left(q^{n-k}-1\right)}>\frac{1}{2} q^{n^{2}-n} .
\end{aligned}
$$

Therefore, (28) gives $N<2 c^{-1} q^{n / 2-1}$. In addition, there are at most $c$ distinct $G$-classes of subgroups of type $\operatorname{Sp}_{n}(q)$ in $G$ (see [33, Proposition 4.8.3]), so there are at most $2 q^{n / 2-1}$ such subgroups in $\mathcal{M}$. Similarly, by applying [33, Proposition 4.8.4], we also find that $\mathcal{M}$ contains at most $2 q^{n / 2-1}$ subgroups of type $O_{n}^{+}(q)$.

To complete the analysis of $\mathcal{M}$, we may assume that $H \in \mathcal{C}_{9}$ is almost simple with socle $H_{0}$. First, assume that $H_{0}=A_{d}$ is an alternating group. (The various possibilities are described in [25, Example 2.6, cases (a)-(c)].) No examples arise in cases (a) and (c) since $H \cap \operatorname{PGL}(V)$ fixes a nondegenerate form on $V$ in each relevant case. In case (b), there are a couple of possibilities when $n=6$ and $d=6$ or 7 . However, [25, Table 3] indicates that $q \geq 7$, so $|y| \geq 13$ by (29), but $|x| \leq 12$ for all $x \in H$, so no examples arise. Similarly, by inspecting [25, Table 5], we can rule out any possibilities with $H_{0}$ a sporadic group.

Finally, suppose that $H_{0}$ is a simple group of Lie type in characteristic $\ell$. If $\ell=p$ is the defining characteristic, then $H$ must appear in [25, Table 6], but there are no relevant cases. Finally, let us assume that $\ell \neq p$. By inspecting [25, Tables 7 and 8], and by considering the corresponding Frobenius-Schur indicators (see [15] and [29]) to determine whether or not $H \cap \operatorname{PGL}(V)$ fixes an appropriate form on $V$, we reduce to the following cases:

\begin{tabular}{|l|l|c|c|l|}
\hline & $H_{0}$ & $\alpha$ & $n$ & $p$ \\
\hline (i) & $\mathrm{PSU}_{4}(3)$ & 28 & 6 & $p \equiv 1(\bmod 6)$ \\
\hline (ii) & $\mathrm{PSL}_{3}(4)$ & 21 & 6 & $p \equiv 1(\bmod 6)$ \\
\hline (iii) & $\mathrm{PSL}_{2}(11)$ & 12 & 6 & $p \neq 2,11$ \\
\hline
\end{tabular}


Here $\alpha$ denotes the maximal order of an element of Aut $\left(H_{0}\right)$. In cases (i) and (ii), we have $|y|>\alpha$ if $p>7$ (see (29)), so we may assume that $G_{0}=$ $\operatorname{PSL}_{6}(7)$. Here $|y| \geq \operatorname{lcm}\left(\left(7^{2}+1\right) / 2,(7+1) / 2\right)=100$, so these cases do not arise. Similarly, in case (iii), we may assume that $q \geq 5($ since $(n, q) \notin \mathcal{B})$; hence, $|y| \geq 13$, and this case can also be discarded.

Let $z \in G$ be an element of prime order. By applying Theorem 2.7 and Proposition 2.8, we deduce that if $(n, q) \notin \mathcal{B}$, then

$$
\alpha(z)<2 q^{k-n}+4 q^{1-n / 2}+((2, q-1)-1) \cdot 4 q^{-n / 2}+2 q^{8-2 n}<1 / 2 .
$$

Moreover, it is straightforward to check that $\alpha(z)<q^{-n / 6}$ if $n>12$ or $q>5$, whence $\alpha(z) \rightarrow 0$ as $|G| \rightarrow \infty$.

Proposition 6.6. Theorem 6.2 holds when $n \equiv 0(\bmod 4)$ and $n \geq 8$.

Proof. If $(n, q)=(8,2)$, then the desired result follows from Proposition 2.17, so we will assume otherwise. By Lemma 6.1, there are three $\mathrm{PGL}_{n}(q)$-classes of involutory graph automorphisms, with representatives $\iota S, \iota S^{+}$, and $\iota S^{-}$, and respective centralizers of type $\operatorname{Sp}_{n}(q), O_{n}^{+}(q)$, and $O_{n}^{-}(q)$. Now $\operatorname{det}\left(S^{+}\right)=(-1)^{n / 2}=1$, so $\iota S$ and $\iota S^{+}$are in the same $G_{0}$-coset, and thus we reduce to the two cases $g=\iota S$ and $g=\iota S^{-}$.

First, assume that $g=\iota S$. This is very similar to the proof of the previous proposition. Set $k=n / 2+2$, so $k$ is even and $(k, n-k)=2$ or 4 . As before, we may take $s \in G_{0}$ such that $y=(g s)^{2}=\left[A^{2}, B^{2}\right]$, where $A \in \operatorname{Sp}_{k}(q)$ and $B \in \operatorname{Sp}_{n-k}(q)$ are irreducible. Then (29) holds, and the subsequent analysis of $\mathcal{M}$ is entirely similar, except that we need an additional argument to eliminate $\mathcal{C}_{6}$ subgroups. Suppose that $H \in \mathcal{M}$ is a $\mathcal{C}_{6}$-subgroup. According to $[25$, Example 2.5$]$, we may assume that $q=p \equiv 1(\bmod 4)$ and that $n=2^{m}$ with $m \geq 3$. Now $H \cap \operatorname{PGL}(V) \leq 2^{2 m}$. $\operatorname{Sp}_{2 m}(2)$, where $2^{2 m}$ is elementary abelian of order $2^{2 m}$, so $|z| \leq 2 \alpha$ for all $z \in H \cap \operatorname{PGL}(V)$, where $\alpha$ is the maximal order of an element of $\operatorname{Sp}_{2 m}(2)$. Clearly, $\alpha \leq 2^{2 m}-1$ (see, e.g., [17, Corollary 2]), so by considering (29), we reduce to the case $(n, q)=(8,5)$. Here $\alpha=15$ and $|y| \geq\left(5^{3}+1\right) / 4$, so there are no $\mathcal{C}_{6}$-subgroups in $\mathcal{M}$. In this way, we deduce that (30) holds, and the result quickly follows. We leave the reader to check the details.

For the remainder of the proof, we may assume that $g=\iota S^{-}$. Here $C_{G_{0}}(g)$ is of type $O_{n}^{-}(q)$, and we set $y=(g s)^{2}=s^{2}$, where $s \in C_{G_{0}}(g)$ is irreducible. Let $\mathcal{M}$ denote the set of maximal subgroups of $G$ containing $g s$. Note that there are no reducible subgroups in $\mathcal{M}$, and also note that some power of 
$y$ has order $r$, where $r$ is a primitive prime divisor of $p^{f n}-1$. (Recall that $q=p^{f}$ and that $p$ is a prime.)

To begin with, let us assume that $(n, q) \neq(12,2)$ or $(20,2)$. Then $[24$, Lemma 2.1] implies that some power of $y$ has order $r^{\prime}$, where either $r^{\prime}>$ $2 n+1$ is a primitive prime divisor of $q^{n}-1$, or $r^{\prime}$ is a product of primitive prime divisors of $q^{n}-1$. Therefore, Theorem 2.12 implies that each $H \in \mathcal{M}$ is of type $\mathrm{GL}_{n / k}\left(q^{k}\right)$ (where $k$ is a prime divisor of $n$ ), $\operatorname{Sp}_{n}(q)$, or $O_{n}^{-}(q)$ (with $q$ odd). Note that there are no subfield subgroups or $\mathcal{C}_{8}$-subgroups of type $\mathrm{GU}_{n}\left(q^{1 / 2}\right)$ in $\mathcal{M}$ since $|H \cap \mathrm{PGL}(V)|$ must be divisible by $r$. Also, since $y$ is irreducible, we deduce that $\mathcal{M}$ contains a unique $\mathcal{C}_{3}$-subgroup of type $\mathrm{GL}_{n / k}\left(q^{k}\right)$ for each prime divisor $k$ of $n$, while the usual argument reveals that there are at most $2 q^{n / 2-1}$ subgroups of type $\operatorname{Sp}_{n}(q)$ or $O_{n}^{-}(q)$ in $\mathcal{M}$. By applying Proposition 2.8 and Corollary 2.9, we deduce that

$$
\begin{aligned}
\alpha(z)< & (\log (n)+1) \cdot f(n, q) \\
& +2 q^{n / 2-1}\left(2 q^{2-n}+((2, q-1)-1) \cdot 2 q^{1-n}\right)<1 / 2
\end{aligned}
$$

for all $z \in G$ of prime order. Moreover, if $n>12$ or $q>4$, then (31) yields $\alpha(z)<q^{-n / 4}$, so the desired asymptotic result also holds.

Finally, suppose that $(n, q)=(12,2)$ or $(20,2)$. By applying the main theorem of [25], we calculate that in both of these cases there are no additional subgroups in $\mathcal{M}$. For example, if $(n, q)=(20,2)$, then $r=41$, and by inspecting [25], we deduce that each $H \in \mathcal{M}$ is of type

$$
\mathrm{GL}_{10}(4), \mathrm{GL}_{4}(32), \mathrm{Sp}_{20}(2) \text {, or } \mathrm{PSL}_{2}(41) \text {. }
$$

Here the last case is a $\mathcal{C}_{9}$-subgroup appearing in [25, Table 8], but we can eliminate it since the Frobenius-Schur indicator of the underlying irreducible representation of $\mathrm{SL}_{2}(41)$ is of minus type (see, e.g., [28, Table 2(b)]); hence, $H$ is contained in a $\mathcal{C}_{8}$-subgroup of type $\operatorname{Sp}_{20}(2)$. The case $(n, q)=$ $(12,2)$ is entirely similar. We conclude that (31) holds, and the result follows.

To complete the proof of Theorem 6.2 , we may assume that $n=4$ or 3 .

Proposition 6.7. Theorem 6.2 holds when $n=4$.

Proof. If $q \leq 9$, then Proposition 2.17 applies, so let us assume that $q \geq 11$. As in the proof of Proposition 6.6, we may assume that $g=\iota S$ or $\iota S^{-}$. In 
both cases, we may choose $s \in G_{0}$ such that $y=(g s)^{2} \in G_{0}$ is irreducible on $V$ and

$$
|y|=\left(q^{2}+1\right) /(2, q-1)
$$

As before, let $\mathcal{M}$ be the set of maximal subgroups of $G$ containing $g s$. Since we are assuming that $q \geq 11,[24$, Lemma 2.1] implies that some power of $y$ has order $r$, where either $r>9$ is a primitive prime divisor of $q^{4}-1$, or $r$ is a product of primitive prime divisors of $q^{4}-1$. Therefore, Theorem 2.12 applies, and we quickly deduce that each $H \in \mathcal{M}$ is of type $\operatorname{GL}_{2}\left(q^{2}\right), \operatorname{Sp}_{4}(q)$, or $O_{4}^{-}(q)$ (with $q$ odd). (Note that $|y|$ does not divide $\left|\mathrm{PGL}_{4}\left(q_{1}\right)\right|$ (where $q=q_{1}^{a}$ for some prime $\left.a\right),\left|\mathrm{PGU}_{4}\left(q^{1 / 2}\right)\right|$, or $\left|O_{4}^{+}(q)\right|$, so these subgroups do not arise.)

Since $y$ is irreducible, the usual argument reveals that $\mathcal{M}$ contains a unique subgroup of type $\mathrm{GL}_{2}\left(q^{2}\right)$. Next, assume that $q$ is odd and that $H$ is of type $O_{4}^{-}(q)$. Set $c=(q-1,4) / 2$, and let $N$ be the number of distinct $G$-conjugates of $H$ containing $y$, so (28) holds. Now

$$
[G: H]=\frac{\left|\mathrm{SL}_{4}(q)\right|}{2 c\left|\mathrm{SO}_{4}^{-}(q)\right|}, \quad\left|y^{G} \cap H\right| \leq \frac{2\left|\mathrm{SO}_{4}^{-}(q)\right|}{q^{2}+1}, \quad\left|y^{G}\right|=\frac{\left|\mathrm{GL}_{4}(q)\right|}{q^{4}-1}
$$

(see [33, Proposition 4.8.4]), and thus $N \leq(q+1) / c$. Since there are at most $c$ distinct $G$-classes of such subgroups, we conclude that there are at most $q+1$ subgroups of type $O_{4}^{-}(q)$ in $\mathcal{M}$.

Now let $N$ be the number of subgroups of type $\operatorname{Sp}_{4}(q)$ in $\mathcal{M}$. We claim that $N \leq(2, q-1)^{3}$. To see this, let $G_{1}=\left\langle\operatorname{PGL}_{4}(q), g\right\rangle$, and observe that all subgroups of $G_{1}$ of type $\operatorname{Sp}_{4}(q)$ are $G_{1}$-conjugate, so we have

$$
N \leq \frac{\left|(g s)^{G_{1}} \cap H\right|}{\left|(g s)^{G_{1}}\right|} \cdot \frac{\left|G_{1}\right|}{|H|}
$$

In order to derive an upper bound on $\left|(g s)^{G_{1}} \cap H\right|$, we are free to assume that $H \leq C_{G_{1}}(\iota S)$. Suppose that $\iota S t_{1}, \iota S t_{2} \in(g s)^{G_{1}} \cap H$, where $t_{1}, t_{2} \in \mathrm{PGL}_{4}(q)$. Now $\left(\iota S t_{1}\right)^{2}=t_{1}^{2}$ and $\left(\iota S t_{2}\right)^{2}=t_{2}^{2}$ are elements of $H \cap \mathrm{PGL}_{4}(q)$ with the same set of eigenvalues, say,

$$
\mathcal{E}=\left\{\lambda, \lambda^{q_{0}}, \lambda^{q_{0}^{2}}=\lambda^{-1}, \lambda^{q_{0}^{3}}=\lambda^{-q_{0}}\right\}
$$

Therefore, it follows that the eigenvalues of $t_{1}$ and $t_{2}$ are either $\left\{\mu, \mu^{q_{0}}, \mu^{q_{0}^{2}}\right.$, $\left.\mu^{q_{0}^{3}}\right\}$ or $\left\{-\mu,(-\mu)^{q_{0}},(-\mu)^{q_{0}^{2}},(-\mu)^{q_{0}^{3}}\right\}$, where $\mu^{2}=\lambda$. But any two irreducible 
semisimple elements in $\mathrm{PGSp}_{4}(q)$ with the same eigenvalues are $\mathrm{PGSp}_{4}(q)$ conjugate. (Since $\mathrm{PGSp}_{4}(q) \cong \mathrm{SO}_{5}(q)$, this follows from [47, Section 2.6]; it can also be deduced from [44, Table 1].) Thus, $t_{1}$ and $t_{2}$ are $\operatorname{PGSp}_{4}(q)$ conjugate, and the same is true for $\iota S t_{1}$ and $\iota S t_{2}$. This implies that $\mid(g s)^{G_{1}} \cap$ $H|\leq(2, q-1)|(g s)^{\mathrm{PGSp}_{4}(q)} \mid$.

Let $m=|g s|$, and observe that $m$ is even and that

$$
C_{\mathrm{PGL}_{4}(q)}(g s) \leq C_{\mathrm{PGL}_{4}(q)}\left((g s)^{m / 2}\right) \cap C_{\mathrm{PGL}_{4}(q)}\left((g s)^{2}\right) .
$$

Now $C_{\mathrm{PGL}_{4}(q)}\left((g s)^{2}\right)$ is cyclic of order $\left(q^{4}-1\right) /(q-1)$, and $(g s)^{m / 2}$ is an involution. Since $C_{\mathrm{PGL}_{4}(q)}\left((g s)^{m / 2}\right)$ contains $(g s)^{2}$, by inspecting the involution classes in the full automorphism group of $G_{0}$, we deduce that $C_{\mathrm{PGL}_{4}(q)}\left((g s)^{m / 2}\right)$ is of type $\mathrm{GL}_{2}\left(q^{2}\right), \mathrm{Sp}_{4}(q)$, or $O_{4}^{-}(q)$. Therefore, $C_{\mathrm{PGL}_{4}(q)}(g s)$ is cyclic of order at most $q^{2}+1$; hence, $\left|C_{G_{1}}(g s)\right| \leq 2\left(q^{2}+1\right)$, and thus

$$
N \leq \frac{(2, q-1)\left|(g s)^{\mathrm{PGSp}_{4}(q)}\right|}{\left|(g s)^{G_{1}}\right|} \cdot \frac{\left|G_{1}\right|}{|H|} \leq \frac{(2, q-1)^{2}\left|C_{G_{1}}(g s)\right|}{\left|C_{H}(g s)\right|} .
$$

But $\left|C_{H}(g s)\right| \geq|g s|=2\left(q^{2}+1\right) /(2, q-1)$ since $H$ contains $g s$, and this yields $N \leq(2, q-1)^{3}$ as claimed.

By applying Lemma 2.11, we conclude that if $z \in G$ has prime order, then

$$
\alpha(z) \leq \frac{d_{2}^{2} q^{2}}{q^{3}-1}+\frac{2 d_{1}\left(d_{2}-1\right)(q+1)}{q^{3}-1}+\frac{d_{1}\left(q^{3}+2 q+1\right)}{q^{2}\left(q^{3}-1\right)}<q^{-1 / 3}
$$

for all $q \geq 11$, where $d_{1}=(4, q-1)$ and $d_{2}=(2, q-1)$.

Proposition 6.8. Theorem 4.1 holds when $n=3$.

Proof. If $q \leq 16$, then Proposition 2.17 applies, so let us assume that $q \geq 17$. We may assume that $g=\iota$ (see Lemma 6.1). Take $s=[A, 1] \in C_{G_{0}}(g)$, where $A \in \mathrm{SO}_{2}^{-}(q)$ is irreducible of order $q+1$, and set $y=(g s)^{2}=\left[A^{2}, 1\right]$. Define $\mathcal{M}$ in the usual way, and note that

$$
|y|=(2, q-1)^{-1}(q+1) .
$$

Clearly, if $H \in \mathcal{M}$ is reducible, then $H$ is of type $\mathrm{GL}_{1}(q) \times \mathrm{GL}_{2}(q)$, and there is a unique such subgroup in $\mathcal{M}$. Now assume that $H \in \mathcal{M}$ is irreducible. We claim that $q$ is odd and that $H$ is of type $O_{3}(q)$.

Suppose that $H \in \mathcal{M}$ is a $\mathcal{C}_{2}$-subgroup of type $\mathrm{GL}_{1}(q)$ \ $S_{3}$. If $x \in H \cap$ $\operatorname{PGL}(V)$, then $|x|$ divides $2(q-1)$ or $3(q-1)$, but this is incompatible 
with (33) since $q \geq 17$. Similarly, since $|y|$ does not divide $\left|\mathrm{PGL}_{3}\left(q_{1}\right)\right|$ or $\left|\mathrm{PGU}_{3}\left(q^{1 / 2}\right)\right|$, we deduce that there are no subfield subgroups or $\mathcal{C}_{8^{-}}$ subgroups of type $\mathrm{GU}_{3}\left(q^{1 / 2}\right)$ in $\mathcal{M}$. We can also eliminate $\mathcal{C}_{3}$-subgroups of type $\mathrm{GL}_{1}\left(q^{3}\right)$ since $|y|$ does not divide $3\left(q^{2}+q+1\right)$. Similarly, if $H$ is a $\mathcal{C}_{6}$-subgroup of type $3^{2} \cdot \operatorname{Sp}_{2}(3)$, then $q=p \equiv 1(\bmod 3)$, so $q \geq 19$ and $|y| \geq 10$, but $|x| \leq 7$ for all $x \in H$. This rules out $\mathcal{C}_{6}$-subgroups.

Finally, suppose that $H \in \mathcal{M}$ is a $\mathcal{C}_{9}$-subgroup with socle $H_{0}$. The possibilities for $H$ are listed in [5, Table 7.4]; either $H_{0}=\mathrm{PSL}_{2}(7)$ or $A_{6}$. Suppose that $H_{0}=\mathrm{PSL}_{2}(7)$, so $q=p \equiv 1,2,4(\bmod 7)$. Here the congruence condition implies that $q \geq 23$, and thus $|y| \geq 12$, which is a contradiction since $|x| \leq 8$ for all $x \in H$. Similar reasoning applies in the case $H_{0}=A_{6}$. This justifies the claim.

Suppose that $g s$ is contained in a subgroup $H$ of type $O_{3}(q)$, where $q$ is odd. Let $N$ be the number of $G$-conjugates of $H$ containing $y=(g s)^{2}$. Let $c=(3, q-1)$. By [33, Proposition 4.8.4], we have $[G: H]=c^{-1}\left[\operatorname{SL}_{3}(q)\right.$ : $\left.\mathrm{SO}_{3}(q)\right]$, and there are at most $c$ distinct $G$-classes of these subgroups in $G$. In addition, we compute

$$
\left|y^{G} \cap H\right|=\left|y^{H}\right|=\frac{\left|\mathrm{SO}_{3}(q)\right|}{\left|\mathrm{GU}_{1}(q)\right|}, \quad\left|y^{G}\right|=\frac{\left|\mathrm{GL}_{3}(q)\right|}{\left|\mathrm{GL}_{1}(q)\right|\left|\mathrm{GL}_{1}\left(q^{2}\right)\right|},
$$

and thus there are at most $q-1$ subgroups of type $O_{3}(q)$ in $\mathcal{M}$.

By applying Theorem 2.7 and Lemma 2.10, we conclude that if $z \in G$ has prime order, then

$$
\alpha(z) \leq q^{-1}+q^{-2}+((2, q-1)-1) \cdot \frac{q-1}{q^{2}+q+1}<2 q^{-1}
$$

for all $q \geq 16$.

This completes the proof of Theorem 6.2. Furthermore, in view of Theorems 3.1, 4.1, and 5.1, the proof of Theorem 2.3 is complete. As explained in Section 2, Theorems 2 and 3 are easily deduced from Theorem 2.3.

\section{$\S 7$. Proof of Corollary 5}

In this final section, we explain how Corollary 5 quickly follows from Theorems 2 and 3. Recall that Corollary 5 states that if $G$ is an almost simple group with socle $G_{0}=\operatorname{PSL}_{n}(q)$, then

$$
d(G)=\max \left\{2, d\left(G / G_{0}\right)\right\} \leq 3,
$$

where $d(L)$ is the minimal size of a generating set for the finite group $L$. 
Write $d=d\left(G / G_{0}\right)$. If $d=1$, then $G / G_{0}$ is cyclic, and thus Theorem 2 implies that $G$ is 2-generated. Next, suppose that $d=2$. Choose $y_{1}, y_{2} \in$ $G$ such that $G=\left\langle G_{0}, y_{1}, y_{2}\right\rangle$, and set $k=\left[\left\langle G_{0}, y_{2}\right\rangle: G_{0}\right]$. Fix $h \in G_{0}$ such that $\left|y_{2} h\right|>k$. (This is possible by the main lemma in [41, Section 2].) By applying Theorem 3 to the group $G_{1}=\left\langle G_{0}, y_{1}\right\rangle$, with $y=\left(y_{2} h\right)^{k} \in G_{0}$ (note that $y \neq 1$ since $\left|y_{2} h\right|>k$ ), we deduce that there exists an element $s \in G_{0}$ and a conjugate $z \in\left(y_{1} s\right)^{G_{1}}$ such that $G_{1}=\langle y, z\rangle$. It follows that $G=\left\langle y_{2} h, z\right\rangle$ is 2-generated.

Finally, suppose that $d=3$, say, $G=\left\langle G_{0}, y_{1}, y_{2}, y_{3}\right\rangle$ for some $y_{1}, y_{2}, y_{3} \in G$. Let $G_{2}=\left\langle G_{0}, y_{1}, y_{2}\right\rangle$. Since $d\left(G_{2} / G_{0}\right)=2$, the above argument implies that there exists $z_{1}, z_{2} \in G_{2}$ such that $G_{2}=\left\langle z_{1}, z_{2}\right\rangle$, and thus $G=\left\langle z_{1}, z_{2}, y_{3}\right\rangle$.

This completes the proof of Corollary 5 .

Acknowledgments. The authors would like to thank Bob Guralnick for bringing this problem to their attention and for many helpful discussions relating to this work. The first author would also like to thank the University of Southern California and the California Institute of Technology for their generous hospitality during a research visit in the spring of 2008 .

\section{REFERENCES}

[1] M. Aschbacher, On the maximal subgroups of the finite classical groups, Invent. Math. 76 (1984), 469-514. MR 0746539. DOI 10.1007/BF01388470.

[2] M. Aschbacher and G. M. Seitz, Involutions in Chevalley groups over fields of even order, Nagoya Math. J. 63 (1976), 1-91. MR 0422401.

[3] G. J. Binder, The two-element bases of the symmetric group, Izv. Vyš̆. Učebn. Zaved. Mat. 90 (1970), 9-11. MR 0257197.

[4] W. Bosma and J. J. Cannon, Handbook of Magma Functions, School of Mathematics and Statistics, University of Sydney, Sydney, 1995.

[5] J. N. Bray, D. F. Holt, and C. M. Roney-Dougal, The Maximal Subgroups of the Low-Dimensional Finite Classical Groups, to appear in London Math. Soc. Lecture Note Ser., Cambridge University Press.

[6] J. L. Brenner and J. Wiegold, Two-generator groups, I, Michigan Math. J. 22 (1975), 53-64. MR 0372033.

[7] T. Breuer, R. M. Guralnick, and W. M. Kantor, Probabilistic generation of finite simple groups, II, J. Algebra 320 (2008), 443-494. MR 2422303. DOI 10.1016/j. jalgebra.2007.10.028.

[8] T. Breuer, R. M. Guralnick, A. Lucchini, A. Maróti, and G. P. Nagy, Hamiltonian cycles in the generating graph of finite groups, Bull. Lond. Math. Soc. 42 (2010), 621-633. MR 2669683. DOI 10.1112/blms/bdq017.

[9] T. C. Burness, Fixed point ratios in actions of finite classical groups, I, J. Algebra 309 (2007), 69-79. MR 2301233. DOI 10.1016/j.jalgebra.2006.05.024.

[10] - Fixed point ratios in actions of finite classical groups, II, J. Algebra 309 (2007), 80-138. MR 2301234. DOI 10.1016/j.jalgebra.2006.05.025. 
[11] - Fixed point ratios in actions of finite classical groups, III, J. Algebra 314 (2007), 693-748. MR 2344583. DOI 10.1016/j.jalgebra.2007.01.011.

[12] - Fixed point ratios in actions of finite classical groups, IV, J. Algebra 314 (2007), 749-788. MR 2344584. DOI 10.1016/j.jalgebra.2007.01.012.

[13] J. J. Cannon and D. F. Holt, Automorphism group computation and isomorphism testing in finite groups, J. Symbolic Comput. 35 (2003), 241-267. MR 1962794. DOI 10.1016/S0747-7171(02)00133-5.

[14] - Computing maximal subgroups of finite groups, J. Symbolic Comput. 37 (2004), 589-609. MR 2094616. DOI 10.1016/j.jsc.2003.08.002.

[15] J. H. Conway, R. T. Curtis, S. P. Norton, R. A. Parker, and R. A. Wilson, Atlas of Finite Groups: Maximal Subgroups and Ordinary Characters for Simple Groups, Oxford University Press, Eynsham, 1985. MR 0827219.

[16] F. Dalla Volta and A. Lucchini, Generation of almost simple groups, J. Algebra $\mathbf{1 7 8}$ (1995), 194-223. MR 1358262. DOI 10.1006/jabr.1995.1345.

[17] M. R. Darafsheh, Orders of elements in the groups related to the general linear group, Finite Fields Appl. 11 (2005), 738-747. MR 2181417. DOI 10.1016/j.ffa.2004.12.003.

[18] J. D. Dixon, The probability of generating the symmetric group, Math. Z. 110 (1969), 199-205. MR 0251758.

[19] J. Fulman and R. Guralnick, Conjugacy class properties of the extension of GL $(n, q)$ generated by the inverse transpose involution, J. Algebra 275 (2004), 356-396. MR 2047453. DOI 10.1016/j.jalgebra.2003.07.004.

[20] D. Gorenstein and R. Lyons, The local structure of finite groups of characteristic 2 type, Mem. Amer. Math. Soc. 42 (1983), no. 276. MR 0690900.

[21] D. Gorenstein, R. Lyons, and R. Solomon, The Classification of the Finite Simple Groups, Math. Surveys Monogr. 40, Amer. Math. Soc., Providence, 1994. MR 1303592.

[22] R. M. Guralnick, The spread of finite groups, in preparation.

[23] R. M. Guralnick and W. M. Kantor, Probabilistic generation of finite simple groups, J. Algebra 234 (2000), 743-792. MR 1800754. DOI 10.1006/jabr.2000.8357.

[24] R. M. Guralnick and G. Malle, Products of conjugacy classes and fixed point spaces, J. Amer. Math. Soc. 25 (2012), 77-121. MR 2833479. DOI 10.1090/ S0894-0347-2011-00709-1.

[25] R. Guralnick, T. Pentilla, C. E. Praeger, and J. Saxl, Linear groups with orders having certain large prime divisors, Proc. Lond. Math. Soc. (3) 78 (1999), 167-214. MR 1658168. DOI 10.1112/S0024611599001616.

[26] R. M. Guralnick and J. Saxl, Generation of finite almost simple groups by conjugates, J. Algebra 268 (2003), 519-571. MR 2009321. DOI 10.1016/S0021-8693(03)00182-0.

[27] R. M. Guralnick and A. Shalev, On the spread of finite simple groups, Combinatorica 23 (2003), 73-87. MR 1996627. DOI 10.1007/s00493-003-0014-3.

[28] G. Hiss and G. Malle, Low-dimensional representations of quasi-simple groups, LMS J. Comput. Math. 4 (2001), 22-63. MR 1835851.

[29] C. Jansen, K. Lux, R. Parker, and R. Wilson, An Atlas of Brauer Characters, London Math. Soc. Monogr. Ser. (N.S.) 11 Oxford University Press, New York, 1995. MR 1367961.

[30] W. M. Kantor, Subgroups of classical groups generated by long root elements, Trans. Amer. Math. Soc. 248, no. 2 (1979), 347-379. MR 0522265. DOI 10.2307/1998972.

[31] W. M. Kantor and A. Lubotzky, The probability of generating a finite classical group, Geom. Dedicata 36 (1990), 67-87. MR 1065213. DOI 10.1007/BF00181465. 
[32] N. Kawanaka, On the irreducible characters of the finite unitary groups, J. Math. Soc. Japan 29 (1977), 425-450. MR 0450383.

[33] P. Kleidman and M. Liebeck, The Subgroup Structure of the Finite Classical Groups, London Math. Soc. Lecture Note Ser. 129, Cambridge University Press, Cambridge, 1990. MR 1057341. DOI 10.1017/CBO9780511629235.

[34] M. W. Liebeck, The classification of finite simple Moufang loops, Math. Proc. Cambridge Philos. Soc. 102 (1987), 33-47. MR 0886433. DOI 10.1017/ S0305004100067025.

[35] M. W. Liebeck and J. Saxl, Minimal degrees of primitive permutation groups, with an application to monodromy groups of covers of Riemann surfaces, Proc. Lond. Math. Soc. (3) 63 (1991), 266-314. MR 1114511. DOI 10.1112/plms/s3-63.2.266.

[36] M. W. Liebeck and A. Shalev, The probability of generating a finite simple group, Geom. Dedicata 56 (1995), 103-113. MR 1338320. DOI 10.1007/BF01263616.

[37] - Classical groups, probabilistic methods, and the (2,3)-generation problem, Ann. of Math. (2) 144 (1996), 77-125. MR 1405944. DOI 10.2307/2118584.

[38] - Simple groups, permutation groups, and probability, J. Amer. Math. Soc. 12 (1999), 497-520. MR 1639620. DOI 10.1090/S0894-0347-99-00288-X.

[39] - Random $(r, s)$-generation of finite classical groups, Bull. Lond. Math. Soc. 34 (2002), 185-188. MR 1874245. DOI 10.1112/S0024609301008827.

[40] F. Lübeck and G. Malle, (2,3)-generation of exceptional groups, J. Lond. Math. Soc. (2) 59 (1999), 109-122. MR 1688493. DOI 10.1112/S002461079800670X.

[41] A. Lucchini and F. Menegazzo, Generators for finite groups with a unique minimal normal subgroup, Rend. Semin. Mat. Univ. Padova 98 (1997), 173-191. MR 1492976.

[42] S. Ramanujan, "A proof of Bertrand's postulate" in Collected Papers of Srinivasa Ramanujan, AMS Chelsea, Providence, 2000, 208-209. MR 2280867.

[43] A. Shalev, Random generation of finite simple groups by p-regular or p-singular elements, Israel J. Math. 125 (2001), 53-60. MR 1853805. DOI 10.1007/BF02773374.

[44] K. Shinoda, The characters of the finite conformal symplectic group, $\operatorname{CSp}(4, q)$, Comm. Algebra 10 (1982), 1369-1419. MR 0662708. DOI 10.1080/ 00927878208822782 .

[45] R. Steinberg, Generators for simple groups, Canad. J. Math. 14 (1962), 277-283. MR 0143801.

[46] - Lectures on Chevalley Groups, Yale University Press, New Haven, 1968. MR 0466335.

[47] G. E. Wall, On the conjugacy classes in the unitary, symplectic and orthogonal groups, J. Aust. Math. Soc. 3 (1963), 1-62. MR 0150210.

[48] K. Zsigmondy, Zur Theorie der Potenzreste, Monatsh. Math. Phys. 3 (1892), 265284. MR 1546236. DOI 10.1007/BF01692444.

\author{
Timothy C. Burness \\ School of Mathematics \\ University of Southampton \\ Southampton SO17 1BJ \\ $U K$ \\ t.burness@soton.ac.uk
}


Simon Guest

School of Mathematics

University of Southampton

Southampton SO17 1BJ

$U K$

s.d.guest@soton.ac.uk 